EDITORIAL BOARD

ANTONIO CARCATERRA

ERIC A. CARLEN

FRANCESCO DELL'ISOLA

RAFFAELE ESPOSITO

ALBERT FANNJIANG

Gilles A. FrancFort

Pierangelo Marcati

JEAN-JACQUES MARIGO

PETER A. MARKOWICH

MARTIN OSTOJA-STARZEWSKI

PIERRE SEPPECHER

DAVID J. STEIGMANN

PAUL STEINMANN

PierRe M. SuQueT

MANAGING EDITORS

MICOL AMAR

ANGELA MADEO

MARTIN OSTOJA-STARZEWSKI

\section{ADVISORY BOARD}

ADNAN AKAY

Holm AltenbaCH

MICOL AMAR

HARM ASKES

TEODOR ATANACKOVIĆ

VICTOR BERDICHEVSKY

GuY BouchitTÉ

ANDREA BRAIDES

ROBERTO CAMASSA

MAURO CARFORE

ERIC DARVE

FELIX DARVE

ANNA DE MASI

Gianpietro Del Piero

Emmanuele Di Benedetto

VICTOR A. EREMEYEV

BERNOLD FIEDLER

IRENE M. GAMBA

DAVID Y. GAO

SERGEy GaVRILYUK

Timothy J. HeAley

DOMINIQUE JEULIN

ROgER E. KHAYAT

CORRADO LATTANZIO

ROBERT P. LIPTON

ANGELO LUONGO

ANGEla MADEO

JUAN J. MANFREDI

CARlo MARCHIORO

ANIL MISRA

ROBERTO NATALINI

PATRIZIO NEFF

Thomas J. Pence

ANDREY PIATNITSKI

ERRICO PRESUTtI

MARIO PUlVIRENTI

Lucio Russo

Miguel A. F. SANJUAN

PATRICK SElvaduraI

MIROSLAV ŠILHAVÝ

GUIDO SWEERS

ANTOINETTE TORDESILLAS

LEV TRUSKINOVSKY

JUAN J. L. VELÁZQUEZ

VINCENZO VESPRI

ANGELO VULPIANI msp.org/memocs

Università di Roma "La Sapienza", Italia

Rutgers University, USA

(CO-CHAIR) Università di Roma "La Sapienza", Italia

(TREASURER) Università dell'Aquila, Italia

University of California at Davis, USA

(CO-CHAIR) Université Paris-Nord, France

Università dell' Aquila, Italy

École Polytechnique, France

DAMTP Cambridge, UK, and University of Vienna, Austria

(CHAIR MANAGING EDITOR) Univ. of Illinois at Urbana-Champaign, USA

Université du Sud Toulon-Var, France

University of California at Berkeley, USA

Universität Erlangen-Nürnberg, Germany

LMA CNRS Marseille, France

Università di Roma "La Sapienza", Italia

Université de Lyon-INSA (Institut National des Sciences Appliquées), France (CHAIR MANAGING EDITOR) Univ. of Illinois at Urbana-Champaign, USA

Carnegie Mellon University, USA, and Bilkent University, Turkey

Otto-von-Guericke-Universität Magdeburg, Germany

Università di Roma "La Sapienza”, Italia

University of Sheffield, UK

University of Novi Sad, Serbia

Wayne State University, USA

Université du Sud Toulon-Var, France

Università di Roma Tor Vergata, Italia

University of North Carolina at Chapel Hill, USA

Università di Pavia, Italia

Stanford University, USA

Institut Polytechnique de Grenoble, France

Università dell' Aquila, Italia

Università di Ferrara and International Research Center MEMOCS, Italia

Vanderbilt University, USA

Gdansk University of Technology, Poland

Freie Universität Berlin, Germany

University of Texas at Austin, USA

Federation University and Australian National University, Australia

Université Aix-Marseille, France

Cornell University, USA

École des Mines, France

University of Western Ontario, Canada

Università dell' Aquila, Italy

Louisiana State University, USA

Università dell'Aquila, Italia

Université de Lyon-INSA (Institut National des Sciences Appliquées), France University of Pittsburgh, USA

Università di Roma "La Sapienza", Italia

University of Kansas, USA

Istituto per le Applicazioni del Calcolo "M. Picone", Italy

Universität Duisburg-Essen, Germany

Michigan State University, USA

Narvik University College, Norway, Russia

Università di Roma Tor Vergata, Italy

Università di Roma “La Sapienza”, Italia

Università di Roma “Tor Vergata", Italia

Universidad Rey Juan Carlos, Madrid, Spain

McGill University, Canada

Academy of Sciences of the Czech Republic

Universität zu Köln, Germany

University of Melbourne, Australia

École Polytechnique, France

Bonn University, Germany

Università di Firenze, Italia

Università di Roma La Sapienza, Italia

MEMOCS (ISSN 2325-3444 electronic, 2326-7186 printed) is a journal of the International Research Center for the Mathematics and Mechanics of Complex Systems at the Università dell'Aquila, Italy.

Cover image: "Tangle” by $\odot$ John Horigan; produced using the Context Free program (contextfreeart.org).

PUBLISHED BY

7 mathematical sciences publishers

nonprofit scientific publishing

http://msp.org/

(C) 2019 Mathematical Sciences Publishers 


\title{
HOMOGENIZATION OF NONLINEAR INEXTENSIBLE PANTOGRAPHIC STRUCTURES BY Г-CONVERGENCE
}

\author{
JeAn-JaCQues Alibert And Alessandro Della Corte
}

\begin{abstract}
We prove the $\Gamma$-convergence of a pantographic microstructured sheet with inextensible fibers to a 2D generalized continuum model. Large deformations considered as geometrical nonlinearities are taken into account, and the $\Gamma$-convergence argument is developed in terms of convergence of measure functionals. We also prove a relative compactness property for the sequence of discrete energy functionals.
\end{abstract}

\section{Introduction}

Pantographic structures can be basically described as microstructured artifacts in which two families of parallel fibers are mechanically connected in such a way that changing the angle between two fibers, belonging to the two different families, costs deformation energy. Here is an image of a possible physical realization of a pantographic structure:

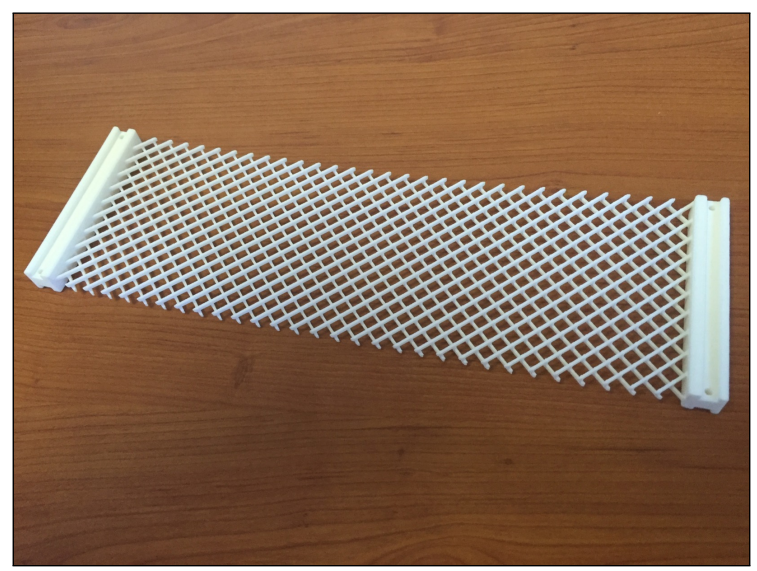

Figure 1. A 3D-printed pantographic sheet made of polyamide (courtesy of Professor Tomasz Lekszycki, Warsaw University of Technology).

\section{Communicated by Pierre Seppecher.}

MSC2010: 46G10, 74B20, 74Q05.

Keywords: $\Gamma$-convergence, nonlinear elasticity, generalized continua, pantographic structures. 
The problem of the equilibrium of lattice structures has been studied for a long time (see for instance [Rivlin 1964; Pipkin 1984; 1986; Steigmann and Pipkin 1991]), and pantographic structures in particular are currently of definite interest both as a structural element, due to their interesting mechanical properties (see for instance [dell'Isola et al. 2016b; Turco et al. 2016b; Battista et al. 2015; Barchiesi et al. 2018b]), and as an experimental and theoretical model case for the onset of behaviors that cannot be described by means of the theory of classical Cauchy continua. In particular it has been shown that generalized continua, in which the energy density depends explicitly on the second gradient of the placement function (see [Mindlin 1964; 1965; Mindlin and Eshel 1968; Germain 1973] for historically important references), are suitable for the description of the deformation of the homogenized version of truss-like [Seppecher et al. 2011; Alibert and Della Corte 2015; Alibert et al. 2003; Turco et al. 2017a] and pantographic structures [Turco et al. 2016a; Rahali et al. 2015].

Here we prove a rigorous homogenization result, namely that a discretized model of pantographic structures (introduced in [dell'Isola et al. 2016a]) $\Gamma$-converges to a homogenized 2D continuum model described by an energy functional in which second partial derivatives of the placement appear. The mathematical study of

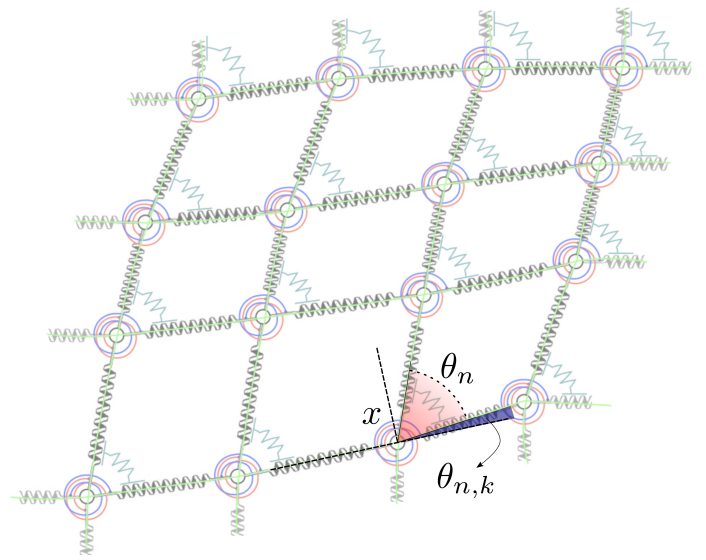

Figure 2. A schematic representation of a pantographic structure in an arbitrary deformed configuration. At every node $x$ of the square lattice there are rotational springs acting between adjacent orthogonal segments (in this case the energy depends, using the notation of Section 3, on the angle $\theta_{n}$ ) and between adjacent parallel segments (in this case the energy depends, using the notation of Section 3 , on the angle $\theta_{n, k}$ ). The nodes are connected by means of extensional springs that in the present paper are particularized to be rigid bars. 
linear pantographic structures is already an active research field (see, e.g., [Boutin et al. 2017; Eremeyev et al. 2018]). In the present paper, the main result will be proven in the large-deformation regime, that is, taking into account geometrical nonlinearities and in particular the actual curvature of the fibers and not only its linearized form.

The microstructure considered herein consists of a square lattice, at each node of which are positioned two types of rotational springs, one acting between adjacent orthogonal segments and the other one acting between adjacent parallel segments (a schematic representation of the structure, in an arbitrary deformed configuration, is shown in Figure 2). In the general case, between the nodes are positioned extensional springs allowing changes in the distances separating adjacent nodes. In the present paper, however, we consider the inextensible case, i.e., we assume that the nodes of the lattice are connected by rigid bars.

The $\Gamma$-convergence argument is developed in terms of convergence of measure functionals. This is, in our opinion, the most sensible approach, since in the real object (at least in planar deformations) most of the deformation energy is actually concentrated in the nodes, stored as torsional deformation energy of the cylindrical pivots interconnecting the two layers of parallel fibers (see, e.g., [Giorgio 2016; dell'Isola et al. 2015]). Therefore, it is quite natural to take this into account in the mathematical modeling introducing a set of vector-valued measures concentrated in the nodes of the lattice. Then we circumscribe the admissible measures by identifying them with functions belonging to suitable Sobolev spaces. This approach allows us to avoid the use of (arbitrary, to some degree) interpolating functions between the nodes. Along with the $\Gamma$-convergence result, we prove a relative compactness property, which ensures that controlling the total deformation energy is enough to control the norm of the measure used for the description of the current configuration of the discrete model.

The paper is organized as follows. In Section 2 the general concept of $\Gamma$ convergence of measure functionals is introduced; in Section 3 the admissible measures are introduced and the energy of the discrete micromodel as well as the boundary conditions are formally described; in Section 4 the same is done for the continuous macromodel and the main result is stated; in Section 5 the main result is proven, including the relative compactness property for the sequence of discrete energy functionals; finally, in Section 6 some conclusions are stated and some possible directions for future studies are indicated.

\section{2. $\Gamma$-convergence of measure functionals}

We start by recalling the definition of $\Gamma$-convergence for a sequence of measure functionals. 
Let $K:=[0,1]^{2}$ and $(C(K))^{2}$ be the space of vector-valued continuous functions on $K$ endowed with the uniform norm $\|\varphi\|_{\infty}:=\sup \{\|\varphi(x)\|: x \in K\}$ where $\|\cdot\|$ denotes the euclidean norm of $\mathbb{R}^{2}$. Let $(M(K))^{2}$ be the set of vector-valued bounded measures on $K$ endowed with the norm

$$
\|\mu\|_{M}:=\sup \left\{\langle\mu, \varphi\rangle: \varphi \in(C(K))^{2},\|\varphi\|_{\infty}=1\right\}
$$

where $\langle\cdot, \cdot\rangle$ stands for the duality bracket between $(M(K))^{2}$ and $\left(C(K)^{2}\right)$. We simply write $\mu_{n} \rightarrow \mu$ to specify that the sequence $\left(\mu_{n}\right)$ of vector bounded measures converges to $\mu$ with respect to the weak* topology, i.e., $\lim _{n \rightarrow \infty}\left\langle\mu_{n}, \varphi\right\rangle=\langle\mu, \varphi\rangle$ for every $\varphi \in(C(K))^{2}$. Recall (see for instance [Evans and Gariepy 2015]) that, if a sequence of vector-valued bounded measures $\left(\mu_{n}\right)$ satisfies

$$
\sup _{n}\left\|\mu_{n}\right\|_{\mathcal{M}}<+\infty
$$

then there exists $\mu \in(M(K))^{2}$ and a subsequence $\left(n_{k}\right)$ such that $\mu_{n_{k}} \rightarrow \mu$. Let $\left(F_{n}\right)$ be a sequence of functionals on $(M(K))^{2}$ with values in $\mathbb{R} \cup\{+\infty\}$. We say that the relative compactness property holds for the sequence $\left(F_{n}\right)$ if for all sequences $\left(\mu_{n}\right)$ in $(M(K))^{2}$

$$
\sup _{n} F_{n}\left(\mu_{n}\right)<+\infty \Rightarrow \sup _{n}\left\|\mu_{n}\right\|_{\mathcal{M}}<+\infty .
$$

We say that the sequence $\left(F_{n}\right) \Gamma$ - converges to $F$ if the following two properties are satisfied.

Lower-bound inequality. For all $\mu \in(\mathcal{M}(K))^{2}$ and all sequences $\left(\mu_{n}\right)$ in $(\mathcal{M}(K))^{2}$

$$
\mu_{n} \rightarrow \mu \quad \Longrightarrow \quad \liminf _{n \rightarrow \infty} F_{n}\left(\mu_{n}\right) \geq F(\mu)
$$

Upper-bound inequality. For each $\mu \in(M(K))^{2}$, there exists a sequence $\left(\mu_{n}\right)$ in $(M(K))^{2}$ such that

$$
\mu_{n} \rightarrow \mu \quad \text { and } \quad \limsup _{n \rightarrow \infty} F_{n}\left(\mu_{n}\right) \leq F(\mu) .
$$

For a general introduction to $\Gamma$-convergence the reader is referred to [Braides 2002].

\section{Micromodel for nonlinear pantographic lattices}

3.1. Reference configuration and basic operators. Let $\delta_{t}$ be the Dirac measure concentrated at the point $t \in[0,1]$. We define four Radon measures on $[0,1]$ by setting

$$
v_{n}:=\frac{1}{n} \sum_{i=0}^{n} \delta_{i / n}, \quad v_{n}^{+}:=\frac{1}{n} \sum_{i=0}^{n-1} \delta_{i / n}, \quad v_{n}^{-}:=\frac{1}{n} \sum_{i=1}^{n} \delta_{i / n}, \quad v_{n}^{2}:=\frac{1}{n} \sum_{i=1}^{n-1} \delta_{i / n} .
$$


The reference configuration of the microsystem is described by the measure $\sigma_{n}:=$ $v_{n} \otimes v_{n}$ on $K:=[0,1] \times[0,1]$. The support of the measure $\sigma_{n}$ is the finite set

$$
\operatorname{Support}\left(\sigma_{n}\right)=\left\{\left(\frac{i}{n}, \frac{j}{n}\right): 0 \leq i, j \leq n\right\}
$$

Each point of the support of $\sigma_{n}$ is called $a$ node. Two nodes $x$ and $y$ are called adjacent when $\|y-x\|=1 / n$ and diagonally adjacent when $\|y-x\|=\sqrt{2} / n$. We define six measures on $K$ and six discrete partial derivative operators by setting

$$
\begin{array}{lll}
\sigma_{n, 1}^{+}:=v_{n}^{+} \otimes v_{n}, & \partial_{n, 1}^{+} u(x):=n\left(u\left(x+\frac{1}{n} e_{1}\right)-u(x)\right), \\
\sigma_{n, 1}^{-}:=v_{n}^{-} \otimes v_{n}, & \partial_{n, 1}^{-} u(x):=n\left(u(x)-u\left(x-\frac{1}{n} e_{1}\right)\right), \\
\sigma_{n, 1}^{2}:=v_{n}^{2} \otimes v_{n}, & \partial_{n, 1}^{2} u(x):=n\left(\partial_{n, 1}^{+} u(x)-\partial_{n, 1}^{-} u(x)\right), \\
\sigma_{n, 2}^{+}:=v_{n} \otimes v_{n}^{+}, & \partial_{n, 2}^{+} u(x):=n\left(u\left(x+\frac{1}{n} e_{2}\right)-u(x)\right), \\
\sigma_{n, 2}^{-}:=v_{n} \otimes v_{n}^{-}, & \partial_{n, 2}^{-} u(x):=n\left(u(x)-u\left(x-\frac{1}{n} e_{2}\right)\right), \\
\sigma_{n, 2}^{2}:=v_{n} \otimes v_{n}^{2}, & \partial_{n, 2}^{2} u(x):=n\left(\partial_{n, 2}^{+} u(x)-\partial_{n, 2}^{-} u(x)\right)
\end{array}
$$

with $e_{1}:=(1,0)$ and $e_{2}:=(0,1)$. Note that, if $u: K \rightarrow \mathbb{R}^{2}$ is defined at every point in the support of $\sigma_{n}$, then for $k \in\{1,2\}$ and $s \in\{+,-, 2\}$ the function $\partial_{n, k}^{s} u$ : $K \rightarrow \mathbb{R}^{2}$ is defined at every point in the support of $\sigma_{n, k}^{s}$. For $a=\left(a_{1}, a_{2}\right) \in \mathbb{R}^{2}$ and $b=\left(b_{1}, b_{2}\right) \in \mathbb{R}^{2}$, we set

$$
a \wedge b:=a_{1} b_{2}-a_{2} b_{1} .
$$

We define four measures on $K$ and four discrete Jacobian determinant operators by setting, for $s, s^{\prime} \in\{+,-\}$

$$
\sigma_{n}^{\left(s, s^{\prime}\right)}:=v_{n}^{s} \otimes v_{n}^{s^{\prime}}, \quad J_{n}^{\left(s, s^{\prime}\right)}(u)(x):=\partial_{n, 1}^{s} u(x) \wedge \partial_{n, 2}^{s^{\prime}} u(x) .
$$

Note that, if $u: K \rightarrow \mathbb{R}^{2}$ is defined at every point in the support of $\sigma_{n}$, then the function $J_{n}^{\left(s, s^{\prime}\right)}(u): K \rightarrow \mathbb{R}$ is defined at every point in the support of $\sigma_{n}^{\left(s, s^{\prime}\right)}$.

3.2. Current configuration and deformation energy of the n-micromodel. The current configuration of the object is described by a vector-valued bounded measure of the special set $\mathcal{M}_{n}$ defined below.

Definition (admissible measures of the $n$-micromodel). The set of admissible measures of the $n$-micromodel is denoted by $\mathcal{M}_{n}$ and consists of those vector bounded measures $\mu \in(M(K))^{2}$ of the form

$$
\mu(d x)=u(x) \sigma_{n}(d x)
$$


where the function $u: K \rightarrow \mathbb{R}^{2}$ is defined at any point $x$ in the support of $\sigma_{n}$ and such that $\left\|\partial_{n, k}^{s} u(x)\right\|>0$ for every $(k, s) \in\{1,2\} \times\{+,-\}$. For any admissible measure $\mu$ of the $n$-micromodel, the following notation is used:

$$
\rho_{n, k}^{s}(\mu)(x):=\left\|\partial_{n, k}^{s} u(x)\right\| \quad \text { and } \quad v_{n, k}^{s}(\mu)(x):=\frac{\partial_{n, k}^{s} u(x)}{\left\|\partial_{n, k}^{s} u(x)\right\|} .
$$

Let $\mu(d x)=u(x) \sigma_{n}(d x) \in \mathcal{M}_{n}$. The function $u$ is called the placement function. The point $u(x)$ is the current position of the node $x$. The above definition of admissible measures imposes the natural requirement that two adjacent nodes should not be mapped by the deformation on the same point (of course this does not exclude the possibility that two generic nodes are mapped on the same point).

At each node $x$ are placed extensional springs which connect $x$ to the adjacent nodes. The deformation energy associated with these extensional springs depends on the distance between the current positions of adjacent nodes and is equal to zero when the distance is equal to $1 / n$. So we introduce the following definition.

Definition (extensional deformation energy of the $n$-micromodel). The extensional deformation energy $E_{n}^{(\mathrm{ext})}$ is defined on $(\mathcal{M}(K))^{2}$ by setting $E_{n}^{(\mathrm{ext})}(\mu)=+\infty$ if $\mu \notin \mu_{n}$ and

$$
E_{n}^{(\mathrm{ext})}(\mu):=\sum_{k=1}^{2} \int f_{k}\left(\rho_{n, k}^{+}(\mu)\right) d \sigma_{n, k}^{+} \text {otherwise, }
$$

where the functions $f_{k}:(0,+\infty) \rightarrow[0,+\infty]$ are assumed to be such that $f_{k}(1)=0$ and $f_{k}(\rho)>0$ if $\rho \neq 1$.

Remark 1. Our main result is obtained in the particular case when the springs between the nodes are just rigid bars, i.e., when $f_{1}$ and $f_{2}$ are the indicator function of the set $\{1\}$ :

$$
f_{k}(\rho):= \begin{cases}0 & \text { if } \rho=1 \\ +\infty & \text { otherwise. }\end{cases}
$$

At each node $x$ are placed four rotational springs (to provide shear stiffness) which connect a pair of segments $\left(\left[x, x+(s / n) e_{1}\right],\left[x, x+\left(s^{\prime} / n\right) e_{2}\right]\right)$ with $s, s^{\prime} \in$ $\{+1,-1\}$. Its energy at the node $x$ depends on the angle $\theta_{n}^{\left(s, s^{\prime}\right)}(\mu)(x)$ formed by the vectors $\partial_{n, 1}^{s} u(x)$ and $\partial_{n, 2}^{s^{\prime}} u(x)$. This energy is equal to zero if and only if the angle is equal to $\pi / 2$. We also assume that angles with finite energy must be in the interval $(0, \pi)$, so as to ensure that nodes diagonally adjacent are not mapped by the deformation on the same point (again, this does not exclude the possibility that two generic nodes are mapped on the same point). One has

$$
\sin \left(\theta_{n}^{\left(s, s^{\prime}\right)}(\mu)(x)\right)=v_{n, 1}^{s}(\mu)(x) \wedge v_{n, 2}^{s^{\prime}}(\mu)(x) .
$$

So we introduce the following definition. 
Definition (first rotational deformation energy of the $n$-micromodel). The first rotational deformation energy $E_{n}^{(\text {shear })}$ is defined on $(\mu(K))^{2}$ by setting $E_{n}^{\text {(shear) }}(\mu)=$ $+\infty$ if $\mu \notin M_{n}$ and

$$
E_{n}^{\text {(shear) }}(\mu):=\sum_{s, s^{\prime} \in\{+,-\}} \int g^{\left(s, s^{\prime}\right)}\left(v_{n, 1}^{s}(\mu) \wedge v_{n, 2}^{s^{\prime}}(\mu)\right) d \sigma_{n}^{\left(s, s^{\prime}\right)} \text { otherwise, }
$$

where the four functions $g^{\left(s, s^{\prime}\right)}:[-1,1] \rightarrow[0,+\infty]$ are assumed to be such that $g^{\left(s, s^{\prime}\right)}(1)=0$.

Remark 2. Our main result is obtained in the particular case when the four functions $g^{\left(s, s^{\prime}\right)}$ are assumed to be lower semicontinuous, convex, and such that $\left\{g^{\left(s, s^{\prime}\right)}<\right.$ $+\infty$ \} is compact and $g^{\left(s, s^{\prime}\right)}(\delta)=+\infty$ if $\delta \leq 0$.

The second type of rotational springs (providing bending rigidity along each coordinate line) is those which connect a pair of segments $\left(\left[x, x+(1 / n) e_{k}\right]\right.$, $\left.\left[x, x-(1 / n) e_{k}\right]\right)$ with $k \in\{1,2\}$. Their energy at the node $x$ depends on the angle $\theta_{n, k}(\mu)(x)$ formed by the vectors $v_{n, k}^{+}(\mu)(x)$ and $v_{n, k}^{-}(\mu)(x)$. This energy is equal to zero if and only if the angle is equal to 0 and one has

$$
1-\cos \left(\theta_{n, k}(\mu)(x)\right)=\frac{1}{2}\left\|v_{n, k}^{+}(\mu)(x)-v_{n, k}^{-}(\mu)(x)\right\|^{2}=\frac{1}{2 n^{2}}\left\|\partial_{n, k}^{-} v_{n, k}^{+}(\mu)(x)\right\|^{2} .
$$

So, we introduce the following definition.

Definition (second rotational deformation energy of the $n$-micromodel). Second rotational deformation energy $E_{n}^{\text {(bend) }}$ is defined on $(\mathcal{M}(K))^{2}$ by setting $E_{n}^{\text {(bend) }}(\mu)=$ $+\infty$ if $\mu \notin \mathcal{M}_{n}$ and

$$
E_{n}^{(\text {bend })}(\mu):=\sum_{k \in\{1,2\}} \int \frac{\kappa_{k}}{2}\left\|\partial_{n, k}^{-} v_{n, k}^{+}(\mu)\right\|^{2} d \sigma_{n, k}^{2} \quad \text { otherwise. }
$$

Remark 3. Our main result is obtained in the case when the two real numbers $\kappa_{k}$ are assumed to be positive (which is quite natural since they represent material coefficients accounting for the bending stiffness of the fibers).

Definition (Dirichlet boundary condition for the $n$-micromodel). Let $\partial K$ denote the boundary of $K$. Let $\Sigma$ be a subset of $\partial K$ and $\mathcal{M}_{n}^{\Sigma}$ be the set of those measures $\mu(d x)=u(x) \sigma_{n}(d x) \in \mathcal{M}_{n}$ such that $u(x)=x$ for every $x \in \Sigma \cap \operatorname{Support}\left(\sigma_{n}\right)$. We denote by $E_{n}^{(\Sigma)}$ the indicator functional of the set $\mu_{n}^{\Sigma}$, i.e.,

$$
E_{n}^{(\Sigma)}(\mu):= \begin{cases}0 & \text { if } \mu \in M_{n}^{\Sigma}, \\ +\infty & \text { otherwise. }\end{cases}
$$

Remark 4. Our main result is obtained in the particular case when $\Sigma:=(a, b) \times\{0\}$ or $\Sigma:=\{0\} \times(a, b)$ with $0 \leq a<b \leq 1$. 


\section{Macromodel and main result}

4.1. Deformation energy of the macromodel. Let $\Omega:=(0,1) \times(0,1)$. For each $p \in[1,+\infty]$ we denote by $L^{p}(\Omega)$ the usual Lebesgue space. Distributional partial derivative operators are denoted by $\partial_{k}$. Distributional second partial derivative operators are denoted by $\partial_{k} \partial_{k^{\prime}}$. If $k=k^{\prime}$, we also write $\partial_{k}^{2}$ in place of $\partial_{k} \partial_{k}$.

Definition (admissible measures of the macromodel). The set of admissible measures is denoted by $M_{\infty}$ and consists of those measures $\mu \in(M(K))^{2}$ of the form

$$
\mu(d x)=u(x) d x
$$

where the function $u: \Omega \rightarrow \mathbb{R}^{2}$ is such that $u \in\left(L^{1}(\Omega)\right)^{2}, \partial_{k} u \in\left(L^{1}(\Omega)\right)^{2}$, $\partial_{k}^{2} u \in\left(L^{2}(\Omega)\right)^{2}$, and $\left\|\partial_{k} u\right\|>0$ a.e. in $\Omega$ for every $k \in\{1,2\}$. For any admissible measure $\mu$, the following notation will be used:

$$
\rho_{k}(\mu):=\left\|\partial_{k} u\right\| \quad \text { and } \quad v_{k}(\mu):=\frac{\partial_{k} u}{\left\|\partial_{k} u\right\|} .
$$

Definition (extensional deformation energy of the macromodel). The extensional deformation energy $E^{(\mathrm{ext})}$ is defined on $(M(K))^{2}$ by setting $E^{(\mathrm{ext})}(\mu)=+\infty$ if $\mu \notin \mu_{\infty}$ and

$$
E^{(\mathrm{ext})}(\mu):=\sum_{k=1}^{2} \int_{\Omega} f_{k}\left(\rho_{k}(\mu)(x)\right) d x \quad \text { otherwise, }
$$

where $f_{k}$ was defined in Remark 1 .

Definition (first rotational deformation energy of the macromodel). The first rotational deformation energy $E^{\text {(shear) }}$ is defined on $(\mu(K))^{2}$ by setting $E^{\text {(shear) }}(\mu)=$ $+\infty$ if $\mu \notin M_{\infty}$ and

$$
E^{(\text {shear })}(\mu):=\int_{\Omega} g\left(v_{1}(\mu)(x) \wedge v_{2}(\mu)(x)\right) d x \text { otherwise, }
$$

where $g:=g^{(+,+)}+g^{(-,+)}+g^{(-,-)}+g^{(+,-)}, g^{s, s^{\prime}}$ was defined in Remark 2, and $v_{k}(\mu)(x)$ was defined in (3).

Definition (second rotational deformation energy of the macromodel). The second rotational deformation energy $E^{\text {(bend) }}$ is defined on $(M(K))^{2}$ by setting $E^{(\text {bend) }}(\mu)=$ $+\infty$ if $\mu \notin M_{\infty}$ and

$$
E^{\text {(bend) }}(\mu):=\sum_{k=1}^{2} \int_{\Omega} \frac{\kappa_{k}}{2}\left\|\partial_{k} v_{k}(\mu)(x)\right\|^{2} d x \quad \text { otherwise. }
$$

If $\mu(d x)=u(x) d x$ is admissible for the macromodel, the placement function $u$ admits a trace on the boundary of $\Omega$ because $u \in\left(L^{1}(\Omega)\right)^{2}$ and $\partial_{k} u \in\left(L^{1}(\Omega)\right)^{2}$ for 
$k=1,2$. The trace function associated with $u$ will be also denoted by $u$ and belongs to the Lebesgue space of integrable functions with respect to the 1D Hausdorff measure supported on $\partial \Omega=\partial K$. This last measure will be denoted by $\mathscr{H}_{\partial \Omega}^{1}$.

Definition (Dirichlet boundary condition for the macromodel). Let $\Sigma$ be a subset of the boundary of $\Omega$ and $\mu_{\infty}^{\Sigma}$ be the set of those measures $\mu(d x)=u(x) d x \in M_{\infty}$ such that $u(x)=x$ for $\mathscr{H}_{\partial \Omega^{1}}^{1}$-a.e. $x \in \Sigma$. We denote by $E^{(\Sigma)}$ the indicator functional of the set $\mu_{\infty}^{\Sigma}$, i.e.,

$$
E^{(\Sigma)}(\mu):= \begin{cases}0 & \text { if } \mu \in \mathcal{M}_{\infty}^{\Sigma} \\ +\infty & \text { otherwise }\end{cases}
$$

4.2. Main result. Our main result states that the total deformation energy of the $n$-micromodel, namely

$$
E_{n}:=E_{n}^{(\text {ext })}+E_{n}^{\text {(shear })}+E_{n}^{\text {(bend) }}+E_{n}^{(\Sigma)},
$$

$\Gamma$-converges to the total deformation energy of the macromodel

$$
E:=E^{(\mathrm{ext})}+E^{(\text {shear })}+E^{(\text {bend })}+E^{(\Sigma)} .
$$

This result is obtained under the assumption that two adjacent nodes are connected by an inextensible bar. This is taken into account by assuming that $f_{1}$ and $f_{2}$ are the indicator function of the set $\{1\}$. Moreover, we need that the angles $\theta_{n}^{\left(s, s^{\prime}\right)}(\mu)$ remain in the interval $(0, \pi)$. Such an assumption is taken into account by assuming that the four functions $g^{\left(s, s^{\prime}\right)}$ are greater than the indicator function of the set $(0,1]$. Finally, we need that the part of the boundary on which the displacement is zero is not too small. More precisely, we have:

Theorem. If we assume that

$\left(\mathrm{H}_{1}\right)$ the two functions $f_{k}:(0,+\infty) \rightarrow[0,+\infty]$ are the indicator function of the set $\{1\}$,

$\left(\mathrm{H}_{2}\right)$ the four functions $g^{\left(s, s^{\prime}\right)}:[-1,1] \rightarrow[0,+\infty]$ are lower semicontinuous, convex, and such that $\left\{g^{\left(s, s^{\prime}\right)}<+\infty\right\}$ is compact, $g^{\left(s, s^{\prime}\right)}(1)=0$, and $g^{\left(s, s^{\prime}\right)}(\delta)=$ $+\infty$ if $\delta \leq 0$,

$\left(\mathrm{H}_{3}\right)$ the two real numbers $\kappa_{k}$ are positive, and

$\left(\mathrm{H}_{4}\right) \quad \Sigma:=(a, b) \times\{0\}$ or $\Sigma:=\{0\} \times(a, b)$ with $0 \leq a<b \leq 1$,

then the sequence $\left(E_{n}\right) \Gamma$-converges to the functional $E$ and the relative compactness property holds. 


\section{Proof of the main theorem}

5.1. Consequences of the assumptions $\left(\mathrm{H}_{1}\right)$ and $\left(\mathrm{H}_{2}\right)$ on placement functions. For the sake of simplicity, in Section 5 we will write $\rho_{n, k}^{+}$and $v_{n, k}^{+}$instead of $\rho_{n, k}^{+}(\mu)$ and $v_{n, k}^{+}(\mu)$.

When property $\left(\mathrm{H}_{1}\right)$ is assumed, the extensional deformation energy of the $n$ micromodel is just the indicator functional of the set of those admissible measures $\mu \in M_{n}$ such that $\rho_{n, k}^{s}(\mu)(x)=1$ for every node $x \in \operatorname{Support}\left(\sigma_{n, k}^{s}\right)$ and every $(k, s) \in\{1,2\} \times\{+,-\}$. Moreover, if $\left(\mathrm{H}_{2}\right)$ is assumed, then measures with finite deformation energy have a very special form.

Lemma 5. If $\left(\mathrm{H}_{1}\right)-\left(\mathrm{H}_{2}\right)$ are assumed and $E_{n}^{(\mathrm{ext})}(\mu)+E_{n}^{(\mathrm{shear})}(\mu)<+\infty$, then the placement function $u$ associated with $\mu$ is such that

$$
\begin{aligned}
\left\|\partial_{n, k}^{+} u(x)\right\|=1 & \text { for every node } x \in \operatorname{Support}\left(\sigma_{n, k}^{+}\right), \\
u(x)+u(0)=\sum_{k=1}^{2} u\left(\left(x \cdot e_{k}\right) e_{k}\right) & \text { for every node } x \in \operatorname{Support}\left(\sigma_{n}\right) .
\end{aligned}
$$

Proof. Since $E_{n}^{(\mathrm{ext})}(\mu)<+\infty$ and $\left(\mathrm{H}_{1}\right)$ is assumed, the first claim of Lemma 5 is clear and for any node $x \in \operatorname{Support}\left(\sigma_{n}^{(+,+)}\right)$one has $v_{n, k}^{+}(x)=\partial_{n, k}^{+} u(x)$. As a consequence,

$$
v_{n, 1}^{+}\left(x+\frac{1}{n} e_{2}\right)-v_{n, 1}^{+}(x)=v_{n, 2}^{+}\left(x+\frac{1}{n} e_{1}\right)-v_{n, 2}^{+}(x) .
$$

Let us denote by $w(x)$ any member of the previous identity. Observing that $\| w(x)+$ $v_{n, 1}^{+}(x) \|^{2}$ and $\left\|w(x)+v_{n, 2}^{+}(x)\right\|^{2}$ are equal to 1 and $v_{n, 2}^{-}\left(x+(1 / n) e_{2}\right)=v_{n, 2}^{+}(x)$, we obtain

$$
\begin{aligned}
w(x) \cdot\left(v_{n, 2}^{+}(x)-v_{n, 1}^{+}(x)\right) & =0, \\
\|w(x)\|^{2}+w(x) \cdot\left(v_{n, 1}^{+}(x)+v_{n, 2}^{+}(x)\right) & =0, \\
\left(w(x)+v_{n, 1}^{+}(x)\right) \wedge v_{n, 2}^{+}(x) & =v_{n, 1}^{+}\left(x+\frac{1}{n} e_{2}\right) \wedge v_{n, 2}^{-}\left(x+\frac{1}{n} e_{2}\right) .
\end{aligned}
$$

As $E_{n}^{\text {(shear) }}(\mu)<+\infty$ and $\left(\mathrm{H}_{2}\right)$ is assumed, $v_{n, 1}^{+}\left(x+(1 / n) e_{2}\right) \wedge v_{n, 2}^{-}\left(x+(1 / n) e_{2}\right)>0$ and $v_{n, 1}^{+}(x) \wedge v_{n, 2}^{+}(x)>0$. As a consequence $\left(v_{n, 1}^{+}(x)+v_{n, 2}^{+}(x), v_{n, 2}^{+}(x)-v_{n, 1}^{+}(x)\right)$ is an orthogonal basis of $\mathbb{R}^{2}$, and for some real number $\lambda$, one has

$$
\begin{aligned}
w(x) & =\lambda\left(v_{n, 1}^{+}(x)+v_{n, 2}^{+}(x)\right), \\
\lambda^{2}+\lambda & =0, \\
(\lambda+1) v_{n, 1}^{+}(x) \wedge v_{n, 2}^{+}(x) & =\left(w(x)+v_{n, 1}^{+}(x)\right) \wedge v_{n, 2}^{+}(x)>0 .
\end{aligned}
$$

We obtain $w(x)=0$. Consequently $v_{n, k}^{+}(x)=v_{n, k}^{+}\left(\left(x \cdot e_{k}\right) e_{k}\right)$ for every $x \in$ $\operatorname{Support}\left(\sigma_{n, k}^{+}\right)$and $\partial_{n, 1}^{+} \partial_{n, 2}^{+} u(y)=0$ for every node $y \in \operatorname{Support}\left(\sigma_{n}^{(+,+)}\right)$. The last 
identity is obtained by observing that for all $x=(i / n, j / n) \in \operatorname{Support}\left(\sigma_{n}\right)$

$$
u(x)+u(0)-\sum_{k=1}^{2} u\left(\left(x \cdot e_{k}\right) e_{k}\right)=\frac{1}{n^{2}} \sum_{q=0}^{i-1} \sum_{p=0}^{j-1} \partial_{n, 1}^{+} \partial_{n, 2}^{+} u\left(\frac{q}{n}, \frac{p}{n}\right) .
$$

5.2. Relative compactness property. The fact that the relative compactness property holds for the sequence $\left(E_{n}\right)$ is a direct consequence of the following result.

Lemma 6. If $\left(\mathrm{H}_{1}\right)$ and $\left(\mathrm{H}_{4}\right)$ are assumed, then the relative compactness property holds for the sequence $\left(E_{n}^{(\mathrm{ext})}+E_{n}^{(\Sigma)}\right)$.

Proof. Without loss of generality, we may assume that there exist two real numbers $a$ and $b$ such that $0 \leq a<b \leq 1$ and $\{0\} \times(a, b) \subset \Sigma$. For all $n$ such that $b-a>1 / n$ we set

$$
\varphi_{n}(t):=\frac{\delta_{t}(a, b)}{v_{n}(a, b)}
$$

Let $\mu \in(M(K))^{2}$ such that $E_{n}^{(\mathrm{ext})}(\mu)+E_{n}^{(\Sigma)}(\mu)<+\infty$. Then the measure $\mu$ is such that $\mu(d x)=u(x) \sigma_{n}(d x)$ with $\left\|\partial_{n, k}^{s} u(x)\right\|=1$ for every node $x \in \operatorname{Support}\left(\sigma_{n}\right)$ and $v(x):=u(x)-x=0$ for every node $x \in\{0\} \times(a, b)$. One has

$$
\|\mu\|_{\mathcal{M}}=\int\|u(x)\| \sigma_{n}(d x) \leq \int\|x\| \sigma_{n}(d x)+\int\|v(x)\| \sigma_{n}(d x)
$$

and $\int \varphi_{n}(t) v_{n}(d t)=1$; then for any node $x=\left(x_{1}, x_{2}\right)$,

$$
v(x)=\int\left(v\left(x_{1}, x_{2}\right)-v\left(x_{1}, t\right)\right) \varphi_{n}(t) v_{n}(d t)+\int\left(v\left(x_{1}, t\right)-v(0, t)\right) \varphi_{n}(t) v_{n}(d t),
$$

which implies

$$
\begin{aligned}
\int\|v(x)\| \sigma_{n}(d x) & \leq 2 \int\left\|\partial_{n, 2}^{+} v\right\| d \sigma_{n, 2}^{+}+\frac{4}{v_{n}(a, b)} \int_{[0,1] \times(a, b)}\left\|\partial_{n, 1}^{+} v\right\| d \sigma_{n, 1}^{+} \\
& \leq 2 \int\left\|\partial_{n, 2}^{+} u-e_{2}\right\| d \sigma_{n, 2}^{+}+\frac{4}{v_{n}(a, b)} \int_{[0,1] \times(a, b)}\left\|\partial_{n, 1}^{+} u-e_{1}\right\| d \sigma_{n, 1}^{+} \\
& \leq 8+2 \int\left\|\partial_{n, 2}^{+} u\right\| d \sigma_{n, 2}^{+}+\frac{4}{v_{n}(a, b)} \int\left\|\partial_{n, 1}^{+} u\right\| d \sigma_{n, 1}^{+} \\
& \leq 12+\frac{8}{v_{n}(a, b)} .
\end{aligned}
$$

Since $\left(v_{n}(a, b)^{-1}\right)$ and $\left(\int\|x\| \sigma_{n}(d x)\right)$ are bounded sequences, the proof is complete. 
5.3. Lower-bound inequality. Throughout this subsection, $\left(\mu_{n}\right)$ is a sequence of measures with bounded deformation energy, which implies $\mu_{n}(d x)=u_{n}(x) \sigma_{n}(d x)$ is an admissible measure for the $n$-micromodel and

$$
\sup _{n} E_{n}\left(\mu_{n}\right)<+\infty
$$

To gain some regularity properties for the placement functions, which will be exploited in the following, it is convenient to introduce a sequence $\bar{\mu}_{n}(d x)=\bar{u}_{n}(x) d x$ of admissible measures for the macromodel which will be called the equivalent sequence because of Lemma 9 below.

Lemma 7 (equivalent sequence). We define a sequence $\bar{\mu}_{n}(d x)=\bar{u}_{n}(x) d x$ in $(M(K))^{2}$ by setting, for all $t \in[0,1]$ and all $x \in K$,

$$
\begin{aligned}
\bar{w}_{n, k}(t): & =\sum_{i=1}^{n-1} \delta_{t}\left(\frac{i}{n}-\frac{1}{2 n}, \frac{i}{n}+\frac{1}{2 n}\right) \partial_{n, k}^{-} v_{n, k}^{+}\left(\frac{i}{n} e_{k}\right), \\
\bar{v}_{n, k}(t): & =v_{n, k}^{+}(0)+\int_{0}^{t} \bar{w}_{n, k}(s) d s, \\
\bar{u}_{n}(x): & =u_{n}(0)+\sum_{k=1}^{2} \int_{0}^{x \cdot e_{k}} \bar{v}_{n, k}(t) d t .
\end{aligned}
$$

We assume that $\left(\mathrm{H}_{1}\right),\left(\mathrm{H}_{2}\right)$, and $\left(\mathrm{H}_{3}\right)$ hold. Then $\bar{u}_{n}$ is $C^{1}(K)$ regular with distributional second partial derivatives in $\left(L^{2}(\Omega)\right)^{2}$. Moreover,

$$
\begin{gathered}
\sum_{k=1}^{2} \int_{\Omega} \frac{\kappa_{k}}{2}\left\|\partial_{k}^{2} \bar{u}_{n}(x)\right\|^{2} d x=E_{n}^{(\text {bend) }}\left(\mu_{n}\right) \quad \text { and } \quad \partial_{1} \partial_{2} \bar{u}_{n}=0 \quad \text { in } \Omega, \\
\sum_{k=1}^{2} \frac{\kappa_{k}}{2}\left\|\partial_{k} \bar{u}_{n}(y)-\partial_{k} \bar{u}_{n}(x)\right\|^{2} \leq\|y-x\| E_{n}^{(\text {bend })}\left(\mu_{n}\right) \quad \text { for every } x, y \in K, \\
\partial_{k} \bar{u}_{n}\left(x+\frac{1}{2 n} e_{k}\right)=v_{n, k}^{+}(x) \quad \text { for every } k \in\{1,2\} \text { and } x \in \operatorname{Support}\left(\sigma_{n, k}^{+}\right) .
\end{gathered}
$$

Proof. In the proof of Lemma 5 we proved that $v_{n, k}^{+}(x)=v_{n, k}^{+}\left(\left(x \cdot e_{k}\right) e_{k}\right)$ for every node $x \in \operatorname{Support}\left(\sigma_{n, k}^{+}\right)$. Then a direct computation gives us

$$
\int_{0}^{1}\left\|\bar{w}_{n, k}(t)\right\|^{2} d t=\frac{n}{n+1} \int\left\|\partial_{n, k}^{-} v_{n, k}^{+}\right\|^{2} d \sigma_{n, k}^{2} .
$$

As a consequence $\bar{v}_{n, k}:[0,1] \rightarrow \mathbb{R}^{2}$ is continuous on $[0,1]$ with distributional derivative in $\left(L^{2}(0,1)\right)^{2}$, which implies that $\bar{u}_{n}: K \rightarrow \mathbb{R}^{2}$ is $C^{1}(K)$ regular with distributional second partial derivatives in $\left(L^{2}(\Omega)\right)^{2}$ and such that $\partial_{1} \partial_{2} \bar{u}_{n}=0$ in $\Omega$ 
and

$$
\int_{\Omega}\left\|\partial_{k}^{2} \bar{u}_{n}(x)\right\|^{2} d x=\int_{\Omega}\left\|\bar{w}_{n, k}\left(x \cdot e_{k}\right)\right\|^{2} d x=\int\left\|\partial_{n, k}^{-} v_{n, k}^{+}\right\|^{2} d \sigma_{n, k}^{2} .
$$

Let $x, y \in K$. One has

$$
\begin{aligned}
\left\|\partial_{k} \bar{u}_{n}(y)-\partial_{k} \bar{u}_{n}(x)\right\|^{2}=\left\|\bar{v}_{n, k}\left(y \cdot e_{k}\right)-\bar{v}_{n, k}\left(x \cdot e_{k}\right)\right\|^{2} & =\left\|\int_{x \cdot e_{k}}^{y \cdot e_{k}} \bar{w}_{n, k}(t) d t\right\|^{2} \\
& \leq\|y-x\| \int_{0}^{1}\left\|\bar{w}_{n, k}(t)\right\|^{2} d t \\
& \leq\|y-x\| \int\left\|\partial_{n, k}^{-} v_{n, k}^{+}\right\|^{2} d \sigma_{n}^{2} .
\end{aligned}
$$

Let $x \in \operatorname{Support}\left(\sigma_{n, k}^{+}\right)$. There exists $i \in\{0, \ldots, n-1\}$ such that $i / n=x \cdot e_{k}$; then

$$
\begin{aligned}
\partial_{k} \bar{u}_{n}\left(x+\frac{1}{2 n} e_{k}\right) & =\bar{v}_{n, k}\left(x \cdot e_{k}+\frac{1}{2 n}\right) \\
& =v_{n, k}^{+}(0)+\sum_{q=1}^{i} \int_{q / n-1 / 2 n}^{q / n+1 / 2 n} \partial_{n, k}^{-} v_{n, k}^{+}\left(\frac{q}{n} e_{k}\right) d t \\
& =v_{n, k}^{+}(0)+\sum_{q=1}^{i}\left(v_{n, k}^{+}\left(\frac{q}{n} e_{k}\right)-v_{n, k}^{+}\left(\frac{q-1}{n} e_{k}\right)\right) \\
& =v_{n, k}^{+}\left(\left(x \cdot e_{k}\right) e_{k}\right)=v_{n, k}^{+}(x) .
\end{aligned}
$$

Lemma 8 (admissibility for the equivalent sequence). We assume that $\left(\mathrm{H}_{1}\right),\left(\mathrm{H}_{2}\right)$, and $\left(\mathrm{H}_{3}\right)$ hold. Then $\bar{\mu}_{n}$ is admissible for the macromodel. More precisely, for all $k \in\{1,2\}$,

$$
\partial_{1} \bar{u}_{n}(x) \wedge \partial_{2} \bar{u}_{n}(x)>0 \quad \text { and } \quad 1 \geq\left\|\partial_{k} \bar{u}_{n}(x)\right\|>0
$$

for every $x \in K$.

Proof. When $x=(i / n, j / n) \in \operatorname{Support}\left(\sigma_{n}^{(+,+)}\right)$, using Lemma 5 and the fact that $\mu_{n}$ is admissible for the micromodel (we recall that $\mu_{n}$ is a sequence of measures with bounded energy), we obtain $v_{n, 1}^{+}\left((i / n) e_{1}\right) \wedge v_{n, 2}^{+}\left((j / n) e_{2}\right)=v_{n, 1}^{+}(x) \wedge v_{n, 2}^{+}(x)>0$. As a consequence, for all $q_{1}, q_{2} \in\{1, \ldots, n-1\}$ and all $\theta_{1}, \theta_{2} \in[0,1]$,

$$
\left(\left(1-\theta_{1}\right) v_{n, 1}^{-}\left(\frac{q_{1}}{n} e_{1}\right)+\theta_{1} v_{n, 1}^{+}\left(\frac{q_{1}}{n} e_{1}\right)\right) \wedge\left(\left(1-\theta_{2}\right) v_{n, 2}^{-}\left(\frac{q_{2}}{n} e_{2}\right)+\theta_{2} v_{n, 2}^{+}\left(\frac{q_{2}}{n} e_{2}\right)\right)>0 \text {. }
$$

Let $x \in K$. When $x \in[1 /(2 n), 1-1 /(2 n)]^{2}$ one has

$$
\frac{q_{k}}{n}-\frac{1}{2 n} \leq x \cdot e_{k} \leq \frac{q_{k}}{n}+\frac{1}{2 n}
$$


for some $q_{1}, q_{2} \in\{1, \ldots, n-1\}$. A direct computation gives $\partial_{k} \bar{u}_{n}(x)=\left(1-\theta_{k}\right) v_{n, k}^{-}\left(\frac{q_{k}}{n} e_{k}\right)+\theta_{k} v_{n, k}^{+}\left(\frac{q_{k}}{n} e_{k}\right)$ with $\theta_{k}:=n\left(x \cdot e_{k}-\left(\frac{q_{k}}{n}-\frac{1}{2 n}\right)\right)$. Since $\theta_{k} \in[0,1]$, we obtain $\partial_{1} \bar{u}_{n}(x) \wedge \partial_{2} \bar{u}_{n}(x)>0$ and $1 \geq\left\|\partial_{k} \bar{u}_{n}(x)\right\|>0$. The proof is easily completed when $x \in K \backslash[1 /(2 n), 1-1 /(2 n)]^{2}$.

Lemma 9 (asymptotic equivalence). Assume that $\left(\mathrm{H}_{1}\right),\left(\mathrm{H}_{2}\right)$, and $\left(\mathrm{H}_{3}\right)$ hold and set

$$
\left\|u_{n}-\bar{u}_{n}\right\|_{L^{\infty}\left(\sigma_{n}\right)}:=\sup \left\{\left\|u_{n}(x)-\bar{u}_{n}(x)\right\|: x \in \operatorname{Support}\left(\sigma_{n}\right)\right\} .
$$

Then

$$
\lim _{n}\left\|u_{n}-\bar{u}_{n}\right\|_{L^{\infty}\left(\sigma_{n}\right)}=0 .
$$

Moreover, if $\sup _{n}\left\|\mu_{n}\right\|_{\mathcal{M}}<+\infty$, then

$$
\sup \left\|u_{n}\right\|_{L^{\infty}\left(\sigma_{n}\right)}<+\infty \text { and } \mu_{n}-\bar{\mu}_{n} \rightarrow 0
$$

where $\left(\bar{\mu}_{n}\right)$ is the equivalent sequence defined in Lemma 7.

Proof. Step 1. Let $x=\left(i_{1} / n, i_{2} / n\right) \in \operatorname{Support}\left(\sigma_{n}\right)$. Using Lemmas 5 and 7 we obtain

$$
\begin{aligned}
\left\|u_{n}(x)-\bar{u}_{n}(x)\right\| & \leq \sum_{k=1}^{2}\left\|u_{n}\left(\left(x \cdot e_{k}\right) e_{k}\right)-u_{n}(0)-\int_{0}^{x \cdot e_{k}} \partial_{k} \bar{u}_{n}\left(t e_{k}\right) d t\right\| \\
& =\sum_{k=1}^{2}\left\|u_{n}\left(\frac{i_{k}}{n} e_{k}\right)-u_{n}(0)-\int_{0}^{i_{k} / n} \partial_{k} \bar{u}_{n}\left(t e_{k}\right) d t\right\| \\
& \leq \sum_{k=1}^{2} \sum_{q=0}^{i_{k}-1} \int_{q / n}^{q / n+1 / n}\left\|\partial_{n, k}^{+} u_{n}\left(\frac{q}{n} e_{k}\right)-\partial_{k} \bar{u}_{n}\left(t e_{k}\right)\right\| d t \\
& =\sum_{k=1}^{2} \sum_{q=0}^{i_{k}-1} \int_{q / n}^{q / n+1 / n}\left\|\partial_{k} \bar{u}_{n}\left(\left(\frac{q}{n}+\frac{1}{2 n}\right) e_{k}\right)-\partial_{k} \bar{u}_{n}\left(t e_{k}\right)\right\| d t \\
& \leq \sum_{k=1}^{2} \sum_{q=0}^{i_{k}-1} \int_{q / n}^{q / n+1 / n}\left|\left(\frac{q}{n}+\frac{1}{2 n}\right)+t\right|^{1 / 2}\left(\int\left\|\partial_{n, k}^{-} v_{n, k}^{+}\right\|^{2} d \sigma_{n, k}^{2}\right)^{1 / 2} d t \\
& \leq \sqrt{\frac{1}{2 n}} \sum_{k=1}^{2}\left(\int\left\|\partial_{n, k}^{-} v_{n, k}^{+}\right\|^{2} d \sigma_{n, k}^{2}\right)^{1 / 2} ;
\end{aligned}
$$

then the first claim of Lemma 9 holds because the sequence $\left(E_{n}^{\text {(bend) }}\left(\mu_{n}\right)\right)$ is bounded and $\left(\mathrm{H}_{3}\right)$ is assumed. 
Step 2. Let $x$ be any node of Support $\left(\sigma_{n}\right)$. Using Lemma 5 we obtain

$$
\begin{aligned}
\left\|u_{n}(x)\right\| & \leq \frac{1}{\sigma_{n}(K)} \int\left\|u_{n}(y)\right\| \sigma_{n}(d y)+\frac{1}{\sigma_{n}(K)} \int\left\|u_{n}(x)-u_{n}(y)\right\| \sigma_{n}(d y) \\
& \leq\left\|\mu_{n}\right\|_{\mathcal{M}}+\frac{2}{\sigma_{n}(K)} \int\|x-y\| \sigma_{n}(d y) \\
& \leq\left\|\mu_{n}\right\|_{\mathcal{M}}+2 \sqrt{2} .
\end{aligned}
$$

It is assumed that the sequence $\left(\left\|\mu_{n}\right\|_{M}\right)$ is bounded; then the second claim of Lemma 9 holds.

Step 3. Let us set $\Omega_{x}:=\left\{y \in K: \max _{k}\left|(y-x) \cdot e_{k}\right|<1 /(2 n)\right\}$, and let $\left|\Omega_{x}\right|$ be the area of $\Omega_{x}$. Observe that for any node $x \in \operatorname{Support}\left(\sigma_{n}\right)$

$$
\left|\Omega_{x}\right|= \begin{cases}1 /\left(n^{2}\right) & \text { if } x \text { is an interior point of } K, \\ 1 /\left(4 n^{2}\right) & \text { if } x \text { is an extreme point of } K, \\ 1 /\left(2 n^{2}\right) & \text { otherwise. }\end{cases}
$$

Let $\varphi \in C(K)^{2}$ be a test function. Since $\varphi \cdot \bar{u}_{n}$ is continuous on $K$ one has

$$
\left|\Omega_{x}\right| \varphi\left(y_{x}\right) \cdot \bar{u}_{n}\left(y_{x}\right)=\int_{\Omega_{x}} \varphi(y) \cdot \bar{u}_{n}(y) d y
$$

for some $y_{x} \in \Omega_{x}$. As a consequence $\left\langle\bar{u}_{n}-u_{n}, \varphi\right\rangle=A_{n}+B_{n}+C_{n}+D_{n}$ with

$$
\begin{aligned}
A_{n} & =\sum_{x \in \operatorname{Support}\left(\sigma_{n}\right)}\left|\Omega_{x}\right| \varphi\left(y_{x}\right) \cdot\left(\bar{u}_{n}\left(y_{x}\right)-\bar{u}_{n}(x)\right), \\
B_{n} & =\sum_{x \in \operatorname{Support}\left(\sigma_{n}\right)}\left|\Omega_{x}\right| \varphi\left(y_{x}\right) \cdot\left(\bar{u}_{n}(x)-u_{n}(x)\right), \\
C_{n} & =\sum_{x \in \operatorname{Support}\left(\sigma_{n}\right)}\left|\Omega_{x}\right|\left(\varphi\left(y_{x}\right)-\varphi(x)\right) \cdot u_{n}(x), \\
D_{n} & =\sum_{x \in \operatorname{Support}\left(\sigma_{n}\right)}\left(\left|\Omega_{x}\right|-\frac{1}{n^{2}}\right) \varphi(x) \cdot u_{n}(x) .
\end{aligned}
$$

By Lemma 8 , the sequence $\left(\bar{u}_{n}\right)$ is uniformly equicontinuous on $K$; therefore, $\lim _{n} A_{n}=0$. By Lemma 9, $\lim _{n}\left\|\bar{u}_{n}-u_{n}\right\|_{L^{\infty}\left(\sigma_{n}\right)}=0$; thus, $\lim _{n} B_{n}=0$. The test function $\varphi$ is uniformly continuous on $K$, and it is assumed that the sequence $\left(\int\left\|u_{n}\right\| d \sigma_{n}\right)$ is bounded; thus, $\lim _{n} C_{n}=0$. Observe that

$$
\left|D_{n}\right| \leq \frac{2 n+1}{n^{2}}\|\varphi\|_{L^{\infty}\left(\sigma_{n}\right)}\left\|u_{n}\right\|_{L^{\infty}\left(\sigma_{n}\right)}
$$

then by Lemma $9, \lim _{n} D_{n}=0$. 
Lemma 10 (convergence of the equivalent sequence). We assume that $\left(\mathrm{H}_{1}\right),\left(\mathrm{H}_{2}\right)$, and $\left(\mathrm{H}_{3}\right)$ hold and $\mu_{n} \rightarrow \mu$. Then the limit measure $\mu$ is of the form $\mu(d x)=$ $u(x) d x$ where $u$ is $C^{1}(K)$ regular with distributional second partial derivatives in $\left(L^{2}(\Omega)\right)^{2}$ and $\partial_{1} \partial_{2} u=0$ in $\Omega$. Moreover, for all $k \in\{1,2\}$

$$
\begin{array}{cl}
\bar{u}_{n} \rightarrow u & \text { with respect to the uniform norm on } K, \\
\partial_{k} \bar{u}_{n} \rightarrow \partial_{k} u & \text { with respect to the uniform norm on } K, \\
\partial_{k}^{2} \bar{u}_{n} \rightarrow \partial_{k}^{2} u & \text { with respect to the weak topology of }\left(L^{2}(\Omega)\right)^{2} .
\end{array}
$$

Proof. Since $\mu_{n} \rightarrow \mu$, the Banach-Steinhaus theorem implies $\sup _{n}\left\|\mu_{n}\right\|_{\mu}<+\infty$. Using Lemma 9, we obtain

$$
\bar{u}_{n}(x) d x \rightarrow \mu(d x) .
$$

Lemmas 7 and 8 imply that $\left(\bar{u}_{n}\right)$ and $\left(\partial_{k} \bar{u}_{n}\right)$ are uniformly equicontinuous on $K$. By the Ascoli theorem, $\bar{u}_{n} \rightarrow u$ and $\partial_{k} \bar{u}_{n} \rightarrow \partial_{k} u$ with respect to the uniform norm on $K$ for some $u \in\left(C^{1}(K)\right)^{2}$. As a consequence $\partial_{k}^{2} \bar{u}_{n} \rightarrow \partial_{k}^{2} u$ in the sense of distributions on $\Omega$. Since by Lemma 7 the sequence $\left(\partial_{k}^{2} \bar{u}_{n}\right)$ is bounded with respect to the $\left(L^{2}(\Omega)\right)^{2}$ norm, the above convergence holds with respect to the weak topology of $\left(L^{2}(\Omega)\right)^{2}$.

Lemma 11 (lower-bound inequalities). We assume that $\left(\mathrm{H}_{1}\right),\left(\mathrm{H}_{2}\right),\left(\mathrm{H}_{3}\right)$, and $\left(\mathrm{H}_{4}\right)$ hold and $\mu_{n} \rightarrow \mu$. Then the measure $\mu(d x)=u(x) d x$ is such that

$$
\begin{aligned}
E^{(\text {ext })}(\mu) & =0, \\
E^{(\text {shear })}(\mu) & \leq \liminf _{n} E_{n}^{\text {(shear) }}\left(\mu_{n}\right), \\
E^{(\text {bend })}(\mu) & \leq \liminf _{n} E_{n}^{\text {(bend) }}\left(\mu_{n}\right) .
\end{aligned}
$$

If moreover $\sup _{n} E_{n}^{(\Sigma)}\left(\mu_{n}\right)<+\infty$, then $E^{(\Sigma)}(\mu)=0$.

Proof. Let $\left(\bar{\mu}_{n}\right)$ be the equivalent sequence associated with $\left(\mu_{n}\right)$.

Step 1. Lemma 7 implies that $\left\|\partial_{k} \bar{u}_{n}\left(x+(1 /(2 n)) e_{k}\right)\right\|=1$ for every node $x \in$ $\operatorname{Support}\left(\sigma_{n, k}^{+}\right)$, and Lemma 10 asserts that $\partial_{k} \bar{u}_{n} \rightarrow \partial_{k} u$ with respect to the uniform norm on $K$. Hence, $\left\|\partial_{k} u(x)\right\|=1$ for every $x \in K$, which implies $E^{(\mathrm{ext})}(\mu)=0$. As a consequence $\mu$ is admissible for the macromodel and

$$
E^{(\text {shear })}(\mu)=\int_{\Omega} g\left(\partial_{1} u(x) \wedge \partial_{2} u(x)\right) d x, \quad E^{(\text {bend })}(\mu)=\sum_{k=1}^{2} \int_{\Omega} \frac{\kappa_{k}}{2}\left\|\partial_{k}^{2} u(x)\right\|^{2} d x .
$$

Step 2. Since the four functions $g^{\left(s, s^{\prime}\right)}:[-1,1] \rightarrow[0,+\infty]$ are lower semicontinuous, there exist four sequences of nonnegative functions $\left(g_{p}^{\left(s, s^{\prime}\right)}\right)$ in $C[0,1]$ such 
that

$$
g_{p+1}^{\left(s, s^{\prime}\right)} \geq g_{p} \quad \text { and } \quad \sup _{p}^{\left(s, s^{\prime}\right)}=g^{\left(s, s^{\prime}\right)} .
$$

Using Lemmas 7 and 10 again, the fact that $\sigma_{n}^{\left(s, s^{\prime}\right)}(d x) \rightarrow d x$, and the monotone convergence theorem, we obtain

$$
\begin{aligned}
& \liminf _{n} E_{n}^{(\text {shear })}\left(\mu_{n}\right):=\liminf _{n} \sum_{s, s^{\prime}} \int g^{\left(s, s^{\prime}\right)}\left(v_{n, 1}^{+}(x) \wedge v_{n, 2}^{+}(x)\right) \sigma_{n}^{\left(s, s^{\prime}\right)}(d x) \\
& \quad \geq \sum_{s, s^{\prime}} \liminf _{n} \int g^{\left(s, s^{\prime}\right)}\left(\partial_{1} \bar{u}_{n}\left(x+\frac{s}{2 n} e_{1}\right) \wedge \partial_{2} \bar{u}_{n}\left(x+\frac{s^{\prime}}{2 n} e_{2}\right)\right) \sigma_{n}^{\left(s, s^{\prime}\right)}(d x) \\
& \quad \geq \sup _{p} \sum_{s, s^{\prime}} \liminf _{n} \int g_{p}^{\left(s, s^{\prime}\right)}\left(\partial_{1} \bar{u}_{n}\left(x+\frac{1}{2 n} e_{1}\right) \wedge \partial_{2} \bar{u}_{n}\left(x+\frac{1}{2 n} e_{2}\right)\right) \sigma_{n}^{\left(s, s^{\prime}\right)}(d x) \\
& \geq \sup _{p} \sum_{s, s^{\prime}} \int_{\Omega} g_{p}^{\left(s, s^{\prime}\right)}\left(\partial_{1} u(x) \wedge \partial_{2} u(x)\right) d x \\
& \geq \int_{\Omega} g\left(\partial_{1} u(x) \wedge \partial_{2} u(x)\right) d x=E^{(\text {shear })}(\mu) .
\end{aligned}
$$

Step 3. Using Lemmas 7 and 10 and remembering that the $\left(L^{2}(\Omega)\right)^{2}$ norm is weak lower semicontinuous we obtain

$$
\liminf _{n} E_{n}^{(\text {bend })}\left(\mu_{n}\right)=\liminf _{n} \sum_{k=1}^{2} \int_{\Omega} \frac{\kappa_{k}}{2}\left\|\partial_{k}^{2} \bar{u}_{n}(x)\right\|^{2} d x \geq E^{(\text {bend })}(\mu) .
$$

Step 4. The condition $\sup _{n} E_{n}^{(\Sigma)}\left(\mu_{n}\right)<+\infty$ says that $u_{n}(x)=x$ for every $x \in$ $\Sigma \cap \operatorname{Support}\left(\sigma_{n}\right)$. Using Lemma 9 (i.e., $\lim _{n}\left\|u_{n}-\bar{u}_{n}\right\|_{L^{\infty}\left(\sigma_{n}\right)}=0$ ) we obtain

$$
\limsup _{n}\left\{\left\|\bar{u}_{n}(x)-x\right\|: x \in \Sigma \cap \operatorname{Support}\left(\sigma_{n}\right)\right\}=0 .
$$

Using Lemma 10 we obtain

$$
\lim \sup \left\{\|u(x)-x\|: x \in \Sigma \cap \operatorname{Support}\left(\sigma_{n}\right)\right\}=0 .
$$

Since $\Sigma=O \cap \partial \Omega$ where $O$ is an open subset of $\mathbb{R}^{2}$ we deduce that $u(x)=x$ at any point of $\Sigma$. Hence, $E^{(\Sigma)}(\mu)=0$.

5.4. Upper-bound inequality. We recall that the upper-bound inequality for the sequence $\left(E_{n}\right)$ means that for each $\mu \in(M(K))^{2}$, there exists a sequence $\left(\mu_{n}\right)$ in $(M(K))^{2}$ such that

$$
\mu_{n} \rightarrow \mu \quad \text { and } \quad \limsup _{n \rightarrow \infty} E_{n}\left(\mu_{n}\right) \leq E(\mu) .
$$

This is easily obtained by means of Lemma 13 below. 
Lemma 12. We assume that $\left(\mathrm{H}_{1}\right),\left(\mathrm{H}_{2}\right)$, and $\left(\mathrm{H}_{3}\right)$ hold and $\mu(d x)=u(x) d x$ is an admissible measure for the macromodel such that $E(\mu)<+\infty$. Then the placement function $u$ is $C^{1}(K)$ regular with distributional second partial derivatives in $\left(L^{2}(\Omega)\right)^{2}$ and for all $x \in K$ and all $k \in\{1,2\}$,

$$
\left\|\partial_{k} u(x)\right\|=1 \quad \text { and } \quad u(x)+u(0)=\sum_{k=1}^{2} u\left(\left(x \cdot e_{k}\right) e_{k}\right) .
$$

As a consequence

$$
\begin{aligned}
E^{(\mathrm{ext})}(\mu) & =0, \\
E^{(\text {shear })}(\mu) & =\int_{\Omega} g\left(\partial_{1} u(x) \wedge \partial_{2} u(x)\right) d x, \\
E^{(\text {bend })}(\mu) & =\int_{\Omega} \sum_{k=1}^{2} \frac{\kappa_{k}}{2}\left\|\partial_{k}^{2} u(x)\right\|^{2} d x .
\end{aligned}
$$

Proof. Step $1\left(H^{2}(\Omega)\right.$ regularity). Let $H^{-1}(\Omega)$ denote the dual space of the usual Sobolev space $H_{0}^{1}(\Omega)$. Since $E^{(\mathrm{ext})}(\mu)<+\infty$ and $\left(\mathrm{H}_{1}\right)$ is assumed, one has $\left\|\partial_{k} u\right\|=\rho_{k}(\mu)=1$ a.e. in $\Omega$ and $\partial_{k} \partial_{k} u=\partial_{k} v_{k}(\mu) \in\left(L^{2}(\Omega)\right)^{2}$ for every $k \in\{1,2\}$. As a consequence

$$
\begin{aligned}
\partial_{1} \partial_{2} u & \in\left(H^{-1}(\Omega)\right)^{2}, \\
\partial_{1}\left(\partial_{1} \partial_{2} u\right) & =\partial_{2}\left(\partial_{1}^{2} u\right)=\partial_{2}\left(\partial_{1} v_{1}(\mu)\right) \in\left(H^{-1}(\Omega)\right)^{2}, \\
\partial_{2}\left(\partial_{1} \partial_{2} u\right) & =\partial_{1}\left(\partial_{2}^{2} u\right)=\partial_{1}\left(\partial_{2} v_{2}(\mu)\right) \in\left(H^{-1}(\Omega)\right)^{2} .
\end{aligned}
$$

A well known result by Necas [Carroll et al. 1966] asserts that, if a distribution and its first distributional derivatives are $H^{-1}(\Omega)$ regular and if $\Omega$ is bounded with Lipschitz boundary, then the distribution is $L^{2}(\Omega)$ regular. Hence, $\partial_{1} \partial_{2} u \in$ $\left(L^{2}(\Omega)\right)^{2}$.

Step $2\left(\partial_{1} \partial_{2} u=0\right.$ a.e. in $\left.\Omega\right)$. Thanks to Step 1 one has

$$
\begin{aligned}
& 2\left(\partial_{1} \partial_{2} u\right) \cdot\left(\partial_{1} u+\partial_{2} u\right)=\partial_{2}\left\|\partial_{1} u\right\|^{2}+\partial_{1}\left\|\partial_{2} u\right\|^{2}=\partial_{2} \rho_{1}(\mu)^{2}+\partial_{1} \rho_{2}(\mu)^{2}=0, \\
& 2\left(\partial_{1} \partial_{2} u\right) \cdot\left(\partial_{2} u-\partial_{1} u\right)=\partial_{1}\left\|\partial_{2} u\right\|^{2}-\partial_{2}\left\|\partial_{1} u\right\|^{2}=\partial_{1} \rho_{2}(\mu)^{2}-\partial_{2} \rho_{1}(\mu)^{2}=0 .
\end{aligned}
$$

Since $E^{\text {(shear) }}(\mu)<+\infty$ and $\left(\mathrm{H}_{3}\right)$ is assumed, one has $\partial_{1} u \wedge \partial_{2} u>0$ a.e. in $\Omega$, which implies that $\left(\partial_{1} u+\partial_{2} u, \partial_{2} u-\partial_{1} u\right)$ is a direct orthogonal basis of $\mathbb{R}^{2}$ a.e. in $\Omega$; therefore, $\partial_{1} \partial_{2} u=0$ a.e. in $\Omega$.

Step $3\left(C^{1}(K)\right.$ regularity). Let $k \in\{1,2\}$. By Step 1, Step 2, and the usual Sobolev embedding theorem one has

$$
\partial_{k} u(x)=\bar{v}_{k}\left(x \cdot e_{k}\right) \quad \text { for a.e. } x \in \Omega
$$


for some $\bar{v}_{k} \in(C[0,1])^{2}$ with distributional derivative in $\left(L^{2}(0,1)\right)^{2}$. As a consequence $u \in\left(C^{1}(K)\right)^{2}$ and the proof is easily completed.

Lemma 13 (approximating sequence). We assume that $\left(\mathrm{H}_{1}\right),\left(\mathrm{H}_{2}\right),\left(\mathrm{H}_{3}\right)$, and $\left(\mathrm{H}_{4}\right)$ hold and $\mu(d x)=u(x) d x$ is an admissible measure for the macromodel such that $E(\mu)<+\infty$. Then there exists a sequence $\mu_{n}(d x)=u_{n}(x) \sigma_{n}(d x)$ such that

$$
\begin{aligned}
E_{n}^{(\Sigma)}\left(\mu_{n}\right) & =E^{(\sigma)}(\mu)=0 & \text { for every integer } n, \\
E_{n}^{(\mathrm{ext})}\left(\mu_{n}\right) & =E^{(\mathrm{ext})}(\mu)=0 & \text { for every integer } n, \\
\mu_{n} & >\mu, & \\
\lim _{n} E_{n}^{(\text {shear })}\left(\mu_{n}\right) & =E^{(\text {shear })}(\mu), & \\
E_{n}^{(\text {bend })}\left(\mu_{n}\right) & \leq E^{\text {(bend) }}(\mu) & \text { for every integer } n .
\end{aligned}
$$

Proof. Step 1 (construction of the sequence $\left(\mu_{n}\right)$ ). It is assumed that $n$ is large enough so that at least two nodes are contained in $\Sigma:=(a, b) \times\{0\}$. As a consequence of Lemma 12, one has $\partial_{1} u\left(t e_{1}\right)=e_{1}$ for every $t \in[a, b]$. It is then possible to define $v_{n}$-a.e. two functions $u_{n, k}:[0,1] \rightarrow \mathbb{R}^{2}$ by setting

$$
u_{n, 1}\left(\frac{i}{n}\right)=\frac{i}{n} e_{1} \quad \text { if } a<\frac{i}{n}<b,
$$

$n\left(u_{n, 1}\left(\frac{i+1}{n}\right)-u_{n, 1}\left(\frac{i}{n}\right)\right)=\partial_{1} u\left(\left(\frac{i}{n}+\frac{1}{2 n}\right) e_{1}\right)$ for every $i \in\{0, \ldots, n-1\}$,

and

$$
\begin{aligned}
u_{n, 2}(0) & =u_{n, 1}(0) \\
n\left(u_{n, 2}\left(\frac{j+1}{n}\right)-u_{n, 2}\left(\frac{j}{n}\right)\right) & =\partial_{2} u\left(\left(\frac{j}{n}+\frac{1}{2 n}\right) e_{2}\right) \quad \text { for every } j \in\{0, \ldots, n-1\} .
\end{aligned}
$$

We finally define $\mu_{n}(d x):=u_{n}(x) \sigma_{n}(d x)$ by setting

$$
u_{n}(x):=-u_{n, 1}(0)+\sum_{k=1}^{2} u_{n, k}\left(x \cdot e_{k}\right)
$$

for every $x$ in the support of $\sigma_{n}$. It follows from the definition of $\mu_{n}$ that $u_{n}(x)=x$ for every node $x \in \Sigma$, and then $E_{n}^{(\Sigma)}\left(\mu_{n}\right)=0$. We have also

$$
\partial_{n, k}^{+} u_{n}(x)=n\left(u_{n, k}\left(x \cdot e_{k}+\frac{1}{n}\right)-u_{n, k}\left(x \cdot e_{k}\right)\right)=\partial_{k} u\left(x+\frac{1}{2 n} e_{k}\right) .
$$

Using Lemma 12, we obtain $\left\|\partial_{n, k}^{+} u_{n}(x)\right\|=1$ for every node $x$ in the support of $\sigma_{n, k}^{+}$; then $E_{n}^{(\mathrm{ext})}\left(\mu_{n}\right)=0$. 
Step 2 (weak convergence of the sequence $\left(\mu_{n}\right)$ ). Let us denote

$$
\varepsilon_{n, k}:=\frac{1}{n} \sum_{q=0}^{n-1}\left\|\partial_{k} u\left(\left(\frac{q}{n}+\frac{1}{2 n}\right) e_{1}\right)-n \int_{q / n}^{(q+1) / n} \partial_{k} u\left(t e_{1}\right) d t\right\| .
$$

By Lemma 12, the placement function $u$ is $C^{1}(K)$ regular and then the sequences $\left(\varepsilon_{n, k}\right)$ converge to 0 as $n$ tends to $\infty$. Since $u_{n, 1}\left(x \cdot e_{1}\right)-u(x)=0$ for some node $x \in \Sigma$ and $u_{n, 1}(0)=u_{n, 2}(0)$, one has

$$
\begin{array}{ll}
\left\|u_{n, 1}\left(\frac{i}{n}\right)-u\left(\frac{i}{n} e_{1}\right)\right\| \leq \varepsilon_{n, 1} \quad \text { for every } i \in\{0, \ldots, n-1\}, \\
\left\|u_{n, 2}\left(\frac{j}{n}\right)-u\left(\frac{j}{n} e_{2}\right)\right\| \leq \varepsilon_{n, 1}+\varepsilon_{n, 2} \quad \text { for every } j \in\{0, \ldots, n-1\} .
\end{array}
$$

Let $\varphi$ be a test function in $C(K)$. Using Lemma 12 and the definition of $u_{n}$, a direct computation gives us

$$
\begin{aligned}
& \left\langle\mu_{n}-\mu, \varphi\right\rangle=\left(\int \varphi(x) \sigma_{n}(d x)\right)\left(u(0)-u_{n, 1}(0)\right) \\
& \quad+\sum_{k=1}^{2} \int \varphi(x)\left(u_{n, k}\left(x \cdot e_{k}\right)-u\left(\left(x \cdot e_{k}\right) e_{k}\right)\right) \sigma_{n}(d x) \\
& \quad+\left(\int_{\Omega} \varphi(x) d x-\int \varphi(x) \sigma_{n}(d x)\right) u(0) \\
& \quad+\sum_{k=1}^{2}\left(\int \varphi(x) u\left(\left(x \cdot e_{k}\right) e_{k}\right) \sigma_{n}(d x)-\int_{\Omega} \varphi(x) u\left(\left(x \cdot e_{k}\right) e_{k}\right) d x\right) ;
\end{aligned}
$$

then

$$
\begin{array}{r}
\left|\left\langle\mu_{n}-\mu, \varphi\right\rangle\right| \leq\left|\int \varphi(x) \sigma_{n}(d x)\right|\left(3 \varepsilon_{n, 1}+\varepsilon_{n, 2}\right)+\left|\int_{\Omega} \varphi(x) d x-\int \varphi(x) \sigma_{n}(d x)\right|\|u(0)\| \\
+\sum_{k=1}^{2}\left\|\int \varphi(x) u\left(\left(x \cdot e_{k}\right) e_{k}\right) \sigma_{n}(d x)-\int_{\Omega} \varphi(x) u\left(\left(x \cdot e_{k}\right) e_{k}\right) d x\right\| .
\end{array}
$$

Since the measure $\sigma_{n}$ weakly converges to the Lebesgue measure on $K$ and the function $x \rightarrow \varphi(x) u\left(\left(x \cdot e_{k}\right) e_{k}\right)$ is continuous on $K$, we obtain

$$
\lim _{n}\left|\left\langle\mu_{n}-\mu, \varphi\right\rangle\right|=0 .
$$

Step 3 (convergence of the sequence $E_{n}^{(\text {shear) }}\left(\mu_{n}\right)$ ). Let $x$ be a node in the support of $\sigma_{n}^{s, s^{\prime}}$. Using the definition of $u_{n}$ we obtain

$$
\partial_{n, 1}^{s} u_{n}(x) \wedge \partial_{n, 2}^{s^{\prime}} u_{n}(x)=\partial_{1} u\left(x+\frac{s}{2 n} e_{1}\right) \wedge \partial_{2} u\left(x+\frac{s^{\prime}}{2 n} e_{2}\right) .
$$


By Lemma 12, the function $u$ is $C^{1}(K)$ regular. Assumption $\left(\mathrm{H}_{2}\right)$ and the fact that $E(\mu)<+\infty$ imply that $u(x) \in\left\{g^{\left(s, s^{\prime}\right)}<+\infty\right\}$ for every $x \in K$ and the function $g^{\left(s, s^{\prime}\right)}$ restricted to the closed set $\left\{g^{\left(s, s^{\prime}\right)}<+\infty\right\}$ is continuous. As a consequence, the function

$$
x \rightarrow g^{\left(s, s^{\prime}\right)}\left(\partial_{1} u(x) \wedge \partial_{2} u(x)\right)
$$

is uniformly continuous on $K$, which implies that

$$
\lim _{n} \int g^{\left(s, s^{\prime}\right)}\left(\partial_{n, 1}^{s} u_{n}(x) \wedge \partial_{n, 2}^{s^{\prime}} u_{n}(x)\right) \sigma_{n}^{\left(s, s^{\prime}\right)}(d x)=\int_{\Omega} g^{\left(s, s^{\prime}\right)}\left(\partial_{1} u(x) \wedge \partial_{2} u(x)\right) d x
$$

so that $\lim _{n} E_{n}^{(\text {shear) }}\left(\mu_{n}\right)=E^{\text {(shear) }}(\mu)$.

Step 4 (upper-bound inequality of the sequence $E_{n}^{\text {(bend) }}\left(\mu_{n}\right)$ ). Let $x$ be a node in the support of $\sigma_{n, k}^{2}$. Using Lemma 12 and the definition of $u_{n}$ we obtain

$$
\partial_{n, k}^{2} u_{n}(x)=n\left(\partial_{k} u\left(\left(x \cdot e_{k}+\frac{1}{2 n}\right) e_{k}\right)-\partial_{k} u\left(\left(x \cdot e_{k}-\frac{1}{2 n}\right) e_{k}\right)\right) ;
$$

then Jensen inequality gives us

$$
\left\|\partial_{n, k}^{2} u_{n}(x)\right\|^{2} \leq n \int_{x \cdot e_{k}-1 /(2 n)}^{x \cdot e_{k}+1 /(2 n)}\left\|\partial_{k}^{2} u\left(t e_{k}\right)\right\|^{2} d t .
$$

Integrating with respect to the measure $\sigma_{n, k}^{2}$ we obtain

$$
\int\left\|\partial_{n, k}^{2} u_{n}(x)\right\|^{2} \sigma_{n, k}^{2}(d x) \leq \frac{n-1}{n} \int_{\Omega}\left\|\partial_{k}^{2} u(x)\right\|^{2} d x,
$$

and therefore, $E_{n}^{\text {(bend) }}\left(\mu_{n}\right) \leq E^{\text {(bend) }}(\mu)$.

\section{Conclusions}

In the present paper we proved the $\Gamma$-convergence of a discrete lattice of rigid bars and rotational springs to a $2 \mathrm{D}$ generalized continuum model, along with a relative compactness property for the sequence of discrete energy functionals. The result is proven taking into account geometrical nonlinearities.

The main result can be generalized in various ways, the most important of which is probably the extension of the $\Gamma$-convergence argument to less restrictive hypotheses on the function $f_{k}$, in particular allowing extensional deformation, i.e., changes in the distances of adjacent nodes. The assumptions on the functions $g^{\left(s, s^{\prime}\right)}$ can also be relaxed in future investigations.

Of course, future mathematical studies have to take into account also the novelties of mechanical nature coming from experimental and numerical results. For instance, worth mentioning are the recent results on the peculiar 3D (out-of-theplane) behavior of pantographic structures (see, e.g., [Steigmann and dell'Isola 
2015; Misra et al. 2018; Giorgio et al. 2017; Barchiesi et al. 2018a]) and the investigation of generalized pantographic sheets with nonstraight or nonorthogonal fibers [Turco et al. 2017b; Giorgio et al. 2016]. These findings will probably require the development of new techniques in order to obtain rigorous homogenization results. Finally, the possibility of oscillations at the lattice level would require more complex homogenization formulas where the extensional, bending, and shear deformation energies may not be uncoupled anymore.

\section{References}

[Alibert and Della Corte 2015] J.-J. Alibert and A. Della Corte, "Second-gradient continua as homogenized limit of pantographic microstructured plates: a rigorous proof", Z. Angew. Math. Phys. 66:5 (2015), 2855-2870.

[Alibert et al. 2003] J.-J. Alibert, P. Seppecher, and F. dell'Isola, "Truss modular beams with deformation energy depending on higher displacement gradients", Math. Mech. Solids 8:1 (2003), $51-73$.

[Barchiesi et al. 2018a] E. Barchiesi, G. Ganzosch, C. Liebold, L. Placidi, R. Grygoruk, and W. H. Müller, "Out-of-plane buckling of pantographic fabrics in displacement-controlled shear tests: experimental results and model validation", Continuum Mech. Therm. (online publication January 2018).

[Barchiesi et al. 2018b] E. Barchiesi, M. Spagnuolo, and L. Placidi, "Mechanical metamaterials: a state of the art", Math. Mech. Solids (online publication February 2018).

[Battista et al. 2015] A. Battista, C. Cardillo, D. Del Vescovo, N. L. Rizzi, and E. Turco, "Frequency shifts induced by large deformations in planar pantographic continua", Nanosci. Technol. 6:2 (2015), 161-178.

[Boutin et al. 2017] C. Boutin, F. dell'Isola, I. Giorgio, and L. Placidi, "Linear pantographic sheets: asymptotic micro-macro models identification”, Math. Mech. Complex Syst. 5:2 (2017), 127-162.

[Braides 2002] A. Braides, $\Gamma$-convergence for beginners, Oxford Lecture Series in Mathematics and its Applications 22, Oxford University, 2002.

[Carroll et al. 1966] R. W. Carroll, P. Grisvard, J. Nečas, G. F. D. Duff, J. Friberg, R. Seeley, and J. Gobert, Équations aux dérivées partielles, Séminaire de Mathématiques 19, Presses de L'Université de Montréal, 1966.

[dell'Isola et al. 2015] F. dell'Isola, T. Lekszycki, M. Pawlikowski, R. Grygoruk, and L. Greco, “Designing a light fabric metamaterial being highly macroscopically tough under directional extension: first experimental evidence", Z. Angew. Math. Phys. 66:6 (2015), 3473-3498.

[dell'Isola et al. 2016a] F. dell'Isola, I. Giorgio, M. Pawlikowski, and N. L. Rizzi, "Large deformations of planar extensible beams and pantographic lattices: heuristic homogenization, experimental and numerical examples of equilibrium", P. Roy. Soc. A 472:2185 (2016), art. id. 20150790.

[dell'Isola et al. 2016b] F. dell'Isola, D. Steigmann, and A. Della Corte, "Synthesis of fibrous complex structures: designing microstructure to deliver targeted macroscale response", Appl. Mech. Rev. 67:6 (2016), art. id. 060804.

[Eremeyev et al. 2018] V. A. Eremeyev, F. dell'Isola, C. Boutin, and D. Steigmann, "Linear pantographic sheets: existence and uniqueness of weak solutions", J. Elasticity 132:2 (2018), 175-196.

[Evans and Gariepy 2015] L. C. Evans and R. F. Gariepy, Measure theory and fine properties of functions, CRC, Boca Raton, FL, 2015. 
[Germain 1973] P. Germain, "The method of virtual power in continuum mechanics, II: microstructure”, SIAM J. Appl. Math. 25:3 (1973), 556-575.

[Giorgio 2016] I. Giorgio, "Numerical identification procedure between a micro-Cauchy model and a macro-second gradient model for planar pantographic structures", Z. Angew. Math. Phys. 67:4 (2016), art. id. 95.

[Giorgio et al. 2016] I. Giorgio, A. Della Corte, F. dell'Isola, and D. J. Steigmann, "Buckling modes in pantographic lattices", C. R. Mecanique 344:7 (2016), 487-501.

[Giorgio et al. 2017] I. Giorgio, N. L. Rizzi, and E. Turco, "Continuum modelling of pantographic sheets for out-of-plane bifurcation and vibrational analysis", Proc. A. 473:2207 (2017), art. id. 20170636.

[Mindlin 1964] R. D. Mindlin, "Micro-structure in linear elasticity", Arch. Rational Mech. Anal. 16 (1964), 51-78.

[Mindlin 1965] R. D. Mindlin, "Second gradient of strain and surface-tension in linear elasticity", Int. J. Solid. Struct. 1:4 (1965), 417-438.

[Mindlin and Eshel 1968] R. D. Mindlin and N. N. Eshel, "On first strain-gradient theories in linear elasticity", Int. J. Solid. Struct. 4:1 (1968), 109-124.

[Misra et al. 2018] A. Misra, T. Lekszycki, I. Giorgio, G. Ganzosch, W. H. Müller, and F. dell' Isola, "Pantographic metamaterials show atypical Poynting effect reversal", Mech. Res. Commun. 89 (2018), 6-10.

[Pipkin 1984] A. C. Pipkin, "Equilibrium of Tchebychev nets", Arch. Rational Mech. Anal. 85:1 (1984), 81-97.

[Pipkin 1986] A. C. Pipkin, "Energy minimization for nets with slack", Quart. Appl. Math. 44:2 (1986), 249-253.

[Rahali et al. 2015] Y. Rahali, I. Giorgio, J. F. Ganghoffer, and F. dell'Isola, "Homogenization à la Piola produces second gradient continuum models for linear pantographic lattices", Internat. J. Engrg. Sci. 97 (2015), 148-172.

[Rivlin 1964] R. S. Rivlin, "Networks of inextensible cords", pp. 51-64 in Nonlinear problems of engineering, edited by W. F. Ames, Academic Press, New York, 1964.

[Seppecher et al. 2011] P. Seppecher, J.-J. Alibert, and F. dell'Isola, "Linear elastic trusses leading to continua with exotic mechanical interactions”, J. Phys. Conf. Ser. 319:1 (2011), art. id. 012018.

[Steigmann and dell'Isola 2015] D. J. Steigmann and F. dell'Isola, "Mechanical response of fabric sheets to three-dimensional bending, twisting, and stretching", Acta Mech. Sin. 31:3 (2015), 373382.

[Steigmann and Pipkin 1991] D. J. Steigmann and A. C. Pipkin, "Equilibrium of elastic nets", Philos. Trans. Roy. Soc. London Ser. A 335:1639 (1991), 419-454.

[Turco et al. 2016a] E. Turco, F. dell'Isola, A. Cazzani, and N. L. Rizzi, "Hencky-type discrete model for pantographic structures: numerical comparison with second gradient continuum models", Z. Angew. Math. Phys. 67:4 (2016), art. id. 85.

[Turco et al. 2016b] E. Turco, M. Golaszewski, A. Cazzani, and N. L. Rizzi, "Large deformations induced in planar pantographic sheets by loads applied on fibers: experimental validation of a discrete Lagrangian model", Mech. Res. Commun. 76 (2016), 51-56.

[Turco et al. 2017a] E. Turco, I. Giorgio, A. Misra, and F. dell'Isola, "King post truss as a motif for internal structure of (meta)material with controlled elastic properties", Roy. Soc. Open Sci. 4:10 (2017), art. id. 171153. 
[Turco et al. 2017b] E. Turco, M. Golaszewski, I. Giorgio, and F. D’Annibale, "Pantographic lattices with non-orthogonal fibres: experiments and their numerical simulations", Compos. Part B Eng. 118 (2017), 1-14.

Received 4 Apr 2018. Revised 1 Oct 2018. Accepted 29 Nov 2018.

JEAN-JACQUES ALIBERT: alibert@univ-tln.fr

Institut de Mathématiques de Toulon, Université de Toulon, La Garde, France

Alessandro Della CoRTE: alessandro.dellacorte@uniroma1.it International Research Center for the Mathematics and Mechanics of Complex Systems, Università degli Studi dell'Aquila, L'Aquila, Italy 


\title{
A NOTE ON COUETTE FLOW OF MICROPOLAR FLUIDS ACCORDING TO ERINGEN'S THEORY
}

\author{
Wilhelm RicKERT, ELENA N. VilCHEVSKAYA \\ AND WOLFGANG H. MÜLler
}

\begin{abstract}
In order to model the flow of liquids with internal rotational degrees of freedom the theory of micropolar fluids according to Eringen is applied. The essentials of the theory are outlined and then specialized to Couette flow. The profiles for linear and angular velocities will be computed, and in particular, we shall also study the rise in temperature due to viscous dissipation, which is frequently ignored by mechanicians. Closed-form solutions for all three fields are derived for different boundary conditions. The question as to how the boundary conditions are realized physically will be discussed.
\end{abstract}

\section{Introduction}

This paper is devoted to a description of the flow of fluids with internal rotational degrees of freedom, for example a blood plasma carrying red blood cells or nematic liquid crystals. The flow behavior of such materials can be described by the theory of Eringen (see [Cowin 1974] or [Eringen 2001]). In a nutshell, Eringen's approach, also known as the micropolar theory of fluids, relies on a consistent use of the complete spin balance and the concept of the conservation of microinertia.

Our aim is to study the Couette flow of such fluids not only from the mechanical point of view, i.e., by calculating the linear and angular velocity fields, but also from a thermodynamic one, namely by studying the generation of a temperature field during the flow due to internal dissipation. This will require us to look at the balances of momentum, spin, and internal energy in combination. The fact that, due to the internal dissipation, Couette flow is not isothermal is frequently ignored by the mechanics community. For example, in [Eringen 2001, §9.7], the analysis is explicitly referred to as related to a "steady, isothermal fluid". There are a few papers that address this issue, namely [Kazakia and Ariman 1971; Riha 1975]

\section{Communicated by Francesco dell'Isola.}

Support of this work by a grant from the Russian Foundation for Basic Research (16-01-00815) is gratefully acknowledged.

MSC2010: 74A35, 74A20.

Keywords: polar materials, theory of constitutive functions, closed-form solutions. 
(pressure-driven flow through a plane-parallel channel) and [Singh 1982] (Couette flow). However, in the last reference extremely general boundary conditions are used, which leads to a wealth of constants that make the final solution most difficult to interpret. For such reasons we will be as general as possible but always attempt to give an intuitive interpretation of the results. We will also investigate the boundary conditions, in particular their physical realization, which will hopefully lead to a broader acceptance of micropolar theory in the engineering community. Finally note that for brevity's sake the theoretical part of our presentation will be limited to the absolute essentials.

\section{The relevant balance equations}

Mathematically speaking, our objective is to determine the fields of (a) linear velocity $\boldsymbol{v}(\boldsymbol{x}, t)$, (b) the angular velocity field (also known as microgyration vector [Eringen 2001, p. 8]) $\boldsymbol{\omega}(\boldsymbol{x}, t)$, and (c) the temperature field $T(\boldsymbol{x}, t)$, in all points $\boldsymbol{x}$ and at all times $t$, within a region of space $\mathscr{B}$ through which incompressible matter of constant mass density $\rho_{0}$ and constant (isotropic) microinertia $J_{0}$ is flowing.

The determination of these fields relies on field equations for the primary fields. The field equations are based on balance laws and need to be complemented by suitable constitutive relations later. In spatial description, putting the substantial time derivatives $\frac{\mathrm{d}}{\mathrm{d} t}$ of the balanceable quantities exclusively on the left-hand side, the relevant balances read as

- balance of mass (incompressibility condition)

$$
0=\nabla \cdot \boldsymbol{v}
$$

- balance of linear momentum

$$
\rho_{0} \frac{\mathrm{d} \boldsymbol{v}}{\mathrm{d} t}=\nabla \cdot \sigma
$$

- balance of spin

$$
\rho_{0} J_{0} \frac{\mathrm{d} \omega}{\mathrm{d} t}=\nabla \cdot \boldsymbol{\mu}+\boldsymbol{\sigma}_{\times}
$$

- and the balance of internal energy

$$
\rho_{0} \frac{\mathrm{d} u}{\mathrm{~d} t}=-\nabla \cdot \boldsymbol{q}+\boldsymbol{\sigma}:(\nabla \otimes \boldsymbol{v}+\mathbf{I} \times \boldsymbol{\omega})+\boldsymbol{\mu}:(\nabla \otimes \boldsymbol{\omega}),
$$

where body forces, body couples, and volumetric heat supply have been neglected. The symbol ":" denotes the outer double scalar product $\boldsymbol{C}: \boldsymbol{D}=C_{i j} D_{i j}$. Moreover, $\boldsymbol{\sigma}$ denotes the (nonsymmetric, second-order) Cauchy stress tensor, and $\boldsymbol{\mu}$ is the (second-order) couple stress tensor. In $\boldsymbol{\sigma}_{\times}$the Gibbsian cross, defined by $(\boldsymbol{a} \otimes \boldsymbol{b})_{\times}=$ $\boldsymbol{a} \times \boldsymbol{b}$, is applied to the (nonsymmetric) stress tensor so that its antisymmetric parts are extracted, $u$ is the specific internal energy, and $\boldsymbol{q}$ is the heat flux. For the 
convenience of the reader all tensorial equations are presented in index notation in Appendix A.

\section{Eringen's continuum theory of micropolar fluids}

Eringen's theory of micropolar solids and fluids is summarized comprehensively in [Eringen 1999; 2001], where all of the original literature can also be found. However, for the purpose of this article it is most beneficial to use the condensed version in [Cowin 1974].

The corresponding balance equations of Eringen's micropolar fluid theory are the ones shown in (1)-(4). In [Cowin 1974] a particular focus is on one-dimensional Taylor-Couette and Poiseuille flow problems of an isotropic incompressible micropolar fluid. Hence, the following constitutive equation for the stress tensor is proposed (also see [Zhilin 2012, p. 258]):

$$
\boldsymbol{\sigma}=-p \mathbf{I}+2 \mu \boldsymbol{D}+2 \tau \boldsymbol{h} \times \mathbf{I},
$$

where $p$ is the pressure which in the incompressible case is an arbitrary scalar that guarantees the incompressibility, $\mu$ is the shear viscosity known from classical Navier-Stokes theory, and $\boldsymbol{D}=\frac{1}{2}\left[(\nabla \otimes \boldsymbol{v})^{\top}+\nabla \otimes \boldsymbol{v}\right]$ is the symmetric part of the velocity gradient. The additional antisymmetric part of the stress tensor consists of the (objective) quantity $\boldsymbol{h}=\boldsymbol{\omega}-\boldsymbol{w}$, which is the difference between the angular velocity $\boldsymbol{\omega}$ and the vorticity vector $\boldsymbol{w}=\frac{1}{2} \nabla \times \boldsymbol{v}$, which are both nonobjective. The factor $\tau$ is another viscosity coefficient characteristic of the Cosserat (or antisymmetric) part in the stress tensor. Note that this form of the stress tensor is different from the one in [Cowin 1974]: the terms proportional to $\nabla \cdot \boldsymbol{v}$ are missing due to the incompressibility condition (1). For the couple stress tensor we write

$$
\boldsymbol{\mu}=\alpha \nabla \cdot \boldsymbol{\omega} \mathbf{I}+\beta\left[(\nabla \otimes \boldsymbol{\omega})^{\top}+\nabla \otimes \boldsymbol{\omega}\right]-\gamma\left[(\nabla \otimes \boldsymbol{\omega})^{\top}-\nabla \otimes \boldsymbol{\omega}\right] .
$$

In principle all viscosities $\mu, \tau, \alpha, \beta$, and $\gamma$ can depend upon temperature, but we will ignore this during our analysis. The similarity between the representations of the two stress measures is evident: we are confronted with a linear theory in gradients of velocity and angular velocity ${ }^{1}$ and a linear dependence on the total angular velocity field $\omega$. If these constitutive equations are inserted into the momentum and into the spin balances, (2) and (3), we obtain the coupled system of PDEs

$$
\begin{aligned}
\rho_{0} \frac{\mathrm{d} \boldsymbol{v}}{\mathrm{d} t} & =-\nabla p+(\mu+\tau) \Delta \boldsymbol{v}+2 \tau(\nabla \otimes \boldsymbol{\omega})_{\times}, \\
\rho_{0} J_{0} \frac{\mathrm{d} \boldsymbol{\omega}}{\mathrm{d} t} & =(\alpha+\beta-\gamma) \nabla \nabla \cdot \boldsymbol{\omega}+(\beta+\gamma) \Delta \boldsymbol{\omega}-4 \tau \boldsymbol{\omega}+2 \tau \nabla \times \boldsymbol{v},
\end{aligned}
$$

${ }^{1}$ Recall that $2 \boldsymbol{w} \times \mathbf{I}=(\nabla \otimes \boldsymbol{v})^{\top}-\nabla \otimes \boldsymbol{v}$. 
where $\Delta$ is the Laplace operator. For a derivation as well as for a comparison with the equations in [Cowin 1974], see Appendix A. In order to evaluate the balance of internal energy (4) for the stationary state we need an expression for the heat flux. Following [Eringen 2001, p. 14] we write

$$
\boldsymbol{q}=-\kappa \nabla T+\lambda(\nabla \otimes \omega)_{\times} .
$$

Here $\kappa$ and $\lambda$ are two heat conduction coefficients, the first one being positive and well known from classical Fourier theory. We now proceed to study the solution to the resulting field equations for the case of one-dimensional stationary Couette flow (hence, we do not have to specify a caloric equation of state) between two infiniteplane parallel plates. Observe already now that in this case the non-Fourier part of (8) will play no role.

\section{Specialization to Couette flow}

We shall now concentrate on the solution of a stationary one-dimensional TaylorCouette flow between two parallel infinite plates separated by a distance $h$ in $\boldsymbol{e}_{y^{-}}$ direction [Pennington and Cowin 2000]: possibilities to initiate the motion of the fluid are the following ones. The top plate is subjected to a constant velocity $v_{x}(y=$ $h)=v_{0}$ and provides a constant angular velocity $\omega_{z}(y=h)=\omega_{\mathrm{t}}$, whereas the bottom plate does not move translationally, $v_{x}(y=0)=0$, while an angular velocity $\omega_{z}(y=0)=\omega_{\mathrm{b}}$ is induced. We will refer to such boundary conditions as "velocitycontrolled" or Dirichlet conditions. They are frequently used in the literature, either in this general form [Łukaszewicz 1999] or in a specialized version (e.g., $\omega_{\mathrm{t}}=$ $\omega_{\mathrm{b}}=0$ [Eringen 2001, p. 17] or $\omega_{\mathrm{t}}=-\omega_{0}, \omega_{\mathrm{b}}=\omega_{0}$ [ ̌̌iha 1975; Singh 1982]). Alternatively we may refer to "force-controlled" boundary conditions if a constant shear stress and moment is applied and then transmitted to the fluid, e.g., on the top plate $\sigma_{y x}(y=h)=t_{x}$ and $\mu_{z y}(y=h)=\mu_{z, \mathrm{t}}$ (see [Condiff and Dahler 1964] or [Łukaszewicz 1999, p. 31]). In both cases the fluid will move in the $\boldsymbol{e}_{x}$-direction. We neglect the influence of the specific body force and of the specific body couples and propose the semi-inverse ansatz for the linear and the angular velocities

$$
\boldsymbol{v}=v(y) \boldsymbol{e}_{x}, \quad \boldsymbol{\omega}=\omega(y) \boldsymbol{e}_{z} .
$$

Insertion into the field equations (7) yields the coupled system of ordinary differential equations

$$
0=(\mu+\tau) \frac{\mathrm{d}^{2} v}{\mathrm{~d} y^{2}}+2 \tau \frac{\mathrm{d} \omega}{\mathrm{d} y}, \quad 0=(\beta+\gamma) \frac{\mathrm{d}^{2} \omega}{\mathrm{d} y^{2}}-4 \tau\left(\omega+\frac{1}{2} \frac{\mathrm{d} v}{\mathrm{~d} y}\right) .
$$

Note that there is no contribution from the pressure term in $(7)_{2}$ because we do not apply a pressure gradient in $\boldsymbol{e}_{x}$-direction. We first consider the case of "prescribed 
velocities"; i.e., we impose boundary conditions

$$
v(y=0)=0, \quad v(y=h)=v_{0}, \quad \omega(y=0)=\omega_{\mathrm{b}}, \quad \omega(y=h)=\omega_{\mathrm{t}} .
$$

In view of our interest in the physical realization of boundary conditions, it should be pointed out that the quantities on the right-hand side, i.e., $0, v_{0}, \omega_{\mathrm{b}}$, and $\omega_{\mathrm{t}}$, are kinematic characteristics of the two plates, which must be "transmitted" somehow to the fluid. How this becomes possible is not a question to be asked in context with the mathematical statement. We will get back to it later in the discussion section of the paper.

This system can be solved by rewriting it as a first-order system (see Appendix B). Then the solutions for the linear and angular velocity read

$$
\begin{aligned}
v & =c_{1}+c_{2} h \bar{y}-c_{3} \frac{N h}{L} \exp (2 N L \bar{y})+c_{4} \frac{N h}{L} \exp (-2 N L \bar{y}), \\
\omega & =-\frac{1}{2} c_{2}+c_{3} \exp (2 N L \bar{y})+c_{4} \exp (-2 N L \bar{y})
\end{aligned}
$$

with the dimensionless height $\bar{y}=y / h$ and dimensionless constants $N$ and $L$ defined by

$$
N=\sqrt{\frac{\tau}{\mu+\tau}}, \quad L=\frac{h}{l}, \quad l=\sqrt{\frac{\beta+\gamma}{\mu}} .
$$

By using the boundary conditions (11) the constants $c_{i}$ can be determined:

$$
\begin{aligned}
& c_{1}=-P\left(v_{0}+h \omega^{*}\right) \frac{\mu_{\mathrm{eff}}}{2 \mu}+\frac{h \omega^{\mathrm{d}}}{2} \frac{N^{2}}{P L^{2}}, \quad c_{2}=\left(\frac{v_{0}}{h}+\omega^{*} P\right) \frac{\mu_{\mathrm{eff}}}{\mu}, \\
& c_{3 / 4}=\left(\frac{N}{L} \mp P\right) \frac{\mu_{\mathrm{eff}}}{\mu}\left[ \pm\left(\frac{1}{P}-1\right) \omega^{\mathrm{d}}+\frac{L}{N h}\left(v_{0}+h \omega^{*}\right)\right], \\
& \omega^{*}=\omega_{\mathrm{t}}+\omega_{\mathrm{b}}, \quad \omega^{\mathrm{d}}=\omega_{\mathrm{t}}-\omega_{\mathrm{b}}, \quad P=\frac{N}{L} \tanh (N L), \quad \frac{\mu_{\mathrm{eff}}}{\mu}=\frac{1}{1-P},
\end{aligned}
$$

where $\mu_{\text {eff }}$ is an effective shear viscosity. With the relations

$$
\begin{aligned}
& \frac{\sinh (N L[2 \bar{y}-1])}{\sinh (N L)}=\frac{N}{P L} \sinh (2 N L \bar{y})-\cosh (2 N L \bar{y}), \\
& \frac{\cosh (N L[2 \bar{y}-1])}{\cosh (N L)}=\cosh (2 N L \bar{y})-\frac{P L}{N} \sinh (2 N L \bar{y}),
\end{aligned}
$$


and after some rearrangements the following closed-form solution to the boundary value problem results:

$$
\begin{gathered}
\frac{v(\bar{y})}{v_{0}}=-\frac{P}{2} \frac{\mu_{\mathrm{eff}}}{\mu}\left(\frac{\sinh (N L[2 \bar{y}-1])}{\sinh (N L)}+1-\frac{2 \bar{y}}{P}\right) \\
-\frac{P \mu_{\mathrm{eff}}}{2 \mu} \frac{h \omega^{*}}{v_{0}}\left(\frac{\sinh (N L[2 \bar{y}-1])}{\sinh (N L)}+1-2 \bar{y}\right) \\
+\frac{N^{2}}{2 P L^{2}} \frac{h \omega^{\mathrm{d}}}{v_{0}}\left(1-\frac{\cosh (N L[2 \bar{y}-1])}{\cosh (N L)}\right), \\
\frac{\omega(\bar{y})}{v_{0} / h}=\frac{1}{2} \frac{h \omega^{\mathrm{d}}}{v_{0}} \frac{\sinh (N L[2 \bar{y}-1])}{\sinh (N L)}+\frac{1}{2} \frac{\mu_{\mathrm{eff}}}{\mu}\left(\frac{\cosh (N L[2 \bar{y}-1])}{\cosh (N L)}-1\right) \\
+\frac{h \omega^{*}}{v_{0}} \frac{\mu_{\mathrm{eff}}}{2 \mu}\left(\frac{\cosh (N L[2 \bar{y}-1])}{\cosh (N L)}-P\right) .
\end{gathered}
$$

In the special case of antagonistic spin boundary conditions, $\omega_{\mathrm{t}}=-\omega_{0}$ and $\omega_{\mathrm{b}}=\omega_{0}$, i.e., $\omega^{*}=0$ and $\omega^{\mathrm{d}}=-2 \omega_{0}$, the solution can be simplified [Cowin 1974; Singh 1982]:

$$
\begin{aligned}
& \frac{v(\bar{y})}{v_{0}}=\frac{1}{2} \frac{\mu_{\mathrm{eff}}}{\mu}\left[2 \bar{y}-P\left(1+\frac{\sinh (N L[2 \bar{y}-1])}{\sinh (N L)}\right)\right] \\
& \quad-\frac{h \omega_{0}}{v_{0}} \frac{1}{P}\left(\frac{N}{L}\right)^{2}\left[1-\frac{\cosh (N L[2 \bar{y}-1])}{\cosh (N L)}\right], \\
& \frac{\omega(\bar{y})}{v_{0} / h}=\frac{1}{2} \frac{\mu_{\mathrm{eff}}}{\mu}\left[\frac{\cosh (N L[2 \bar{y}-1])}{\cosh (N L)}-1\right]-\frac{h \omega_{0}}{v_{0}} \frac{\sinh (N L[2 \bar{y}-1])}{\sinh (N L)} .
\end{aligned}
$$

It seems natural to interpret the number $l$, which is given by a ratio of the viscosities occurring in the constitutive equations for the Cauchy and for the couple stress tensors, as a length scale characteristic of the particles on the mesoscale. It is therefore also known as the micropolar length scale parameter in the literature; see for micropolar liquids, e.g., [Cowin 1974], and in an analogous manner also for micropolar solids, see [Zueger and Lakes 2016] as a recent example or [Gauthier and Jahsman 1975], where similar parameters were defined. Similarly, the dimensionless number $N$, the so-called coupling coefficient, is customary and, in our opinion, should preferably be used.

Obviously, we have $0 \leq N \leq 1$, where the zero characterizes traditional NavierStokes flow and values that are close to one stand for a strong influence of the antisymmetric part of the stress tensor. Alternatively we may say that if $N$ approaches zero, the impact of the Cosserat term in the Cauchy stress tensor vanishes and the coupling between linear and angular velocity becomes obsolete.

On the other hand, $0 \leq L \leq \infty$, where $L$ is (through $l$ ) defined by a ratio of new viscosity coefficients and the standard shear viscosity. Also recall that the kinetic 


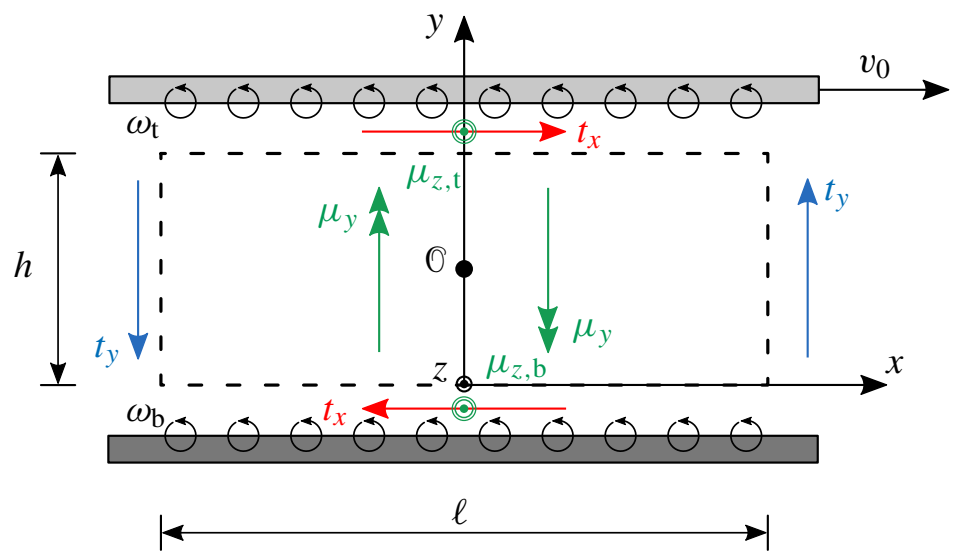

Figure 1. Free body diagram of a section of the Couette flow.

theory of gases allows us to relate the ordinary shear viscosity to the mean free path of molecules [Chapman and Cowling 1952, p. 100]. Therefore, it seems fair to say that $L$-values close to zero indicate a strong influence of characteristic lengths on the mesoscopic scale. In the limiting case where $L$ tends to zero one has

$$
\lim _{L \rightarrow 0} v(\bar{y})=v_{0} \bar{y}+\bar{y}(1-\bar{y}) N^{2} h \omega^{\mathrm{d}}, \quad \lim _{L \rightarrow 0} \omega(\bar{y})=\frac{1}{2}\left(\omega^{*}+\omega^{\mathrm{d}}[2 \bar{y}-1]\right) .
$$

Most interestingly, the linear velocity strongly depends on the boundary conditions of $\omega$, but the angular velocity does not depend on the plate velocity, $v_{0}$. In summary we may say that the Navier-Stokes fluid is obtained if $N \rightarrow 0, L \rightarrow \infty$, and $P \rightarrow 0$. Obviously in this specialized form Eringen's theory has a huge benefit from the viewpoint of the experimentalist: the various new coefficients can be included in only two dimensionless quantities, $N$ and $L$.

It should be noted that Singh [1982] does not define the helpful numbers $L$ and $N$ separately. Rather he combines them in a less intuitive coefficient instead, $\lambda=N L .^{2}$ His solution agrees with ours shown in (15), but it is harder to interpret, in particular, if the intention is not to focus on a special micropolar medium (as Singh does) but to study the impact of the new parameters in general.

We now proceed to discuss the force-controlled experiment. To this end it is useful to consider the free body diagram of the Couette flow shown in Figure 1. Note that the cuts at the upper and lower plates were moved slightly into the fluid region, which enables us to depict the various forces and moments clearly. Of course these cuts are supposed to be flush to the plate surfaces: it is assumed that prescribed forces and moments are transmitted in full from the plate surfaces onto the fluid flanks.

\footnotetext{
${ }^{2}$ Besides that he uses a second coefficient $\xi=2 N^{2}$.
} 


\begin{tabular}{|c|c|c|c|}
\hline flank & $\begin{array}{c}\text { normal } \\
\boldsymbol{n}\end{array}$ & $\begin{array}{l}\text { force traction } \\
\qquad n \cdot \sigma\end{array}$ & $\begin{array}{c}\text { moment traction } \\
\boldsymbol{n} \cdot \boldsymbol{\mu}\end{array}$ \\
\hline $\begin{array}{c}\text { top } \\
\text { bottom }\end{array}$ & $\begin{array}{l}+\boldsymbol{e}_{y} \\
-\boldsymbol{e}_{y}\end{array}$ & $\begin{array}{l}\boldsymbol{t}_{x}=\mu_{\mathrm{eff}}\left(v_{0} / h+P \omega^{*}\right) \boldsymbol{e}_{x} \\
\boldsymbol{t}_{x}=-\mu_{\mathrm{eff}}\left(v_{0} / h+P \omega^{*}\right) \boldsymbol{e}_{x}\end{array}$ & $\begin{aligned} \boldsymbol{\mu}_{z, \mathrm{t}}= & \left(\mu_{\mathrm{eff}} P\left[v_{0}+h \omega^{*}\right]\right. \\
& \left.+\left(\mu N^{2} /\left(P L^{2}\right)\right) h \omega^{\mathrm{d}}\right) \boldsymbol{e}_{z} \\
\boldsymbol{\mu}_{z, \mathrm{~b}}= & \left(\mu_{\mathrm{eff}} P\left[v_{0}+h \omega^{*}\right]\right. \\
& \left.-\left(\mu N^{2} /\left(P L^{2}\right)\right) h \omega^{\mathrm{d}}\right) \boldsymbol{e}_{z}\end{aligned}$ \\
\hline $\begin{array}{l}\text { right } \\
\text { left }\end{array}$ & $\begin{array}{l}+\boldsymbol{e}_{x} \\
-\boldsymbol{e}_{x}\end{array}$ & $\begin{array}{l}\boldsymbol{t}_{y}=\mu_{\mathrm{eff}}\left([1-2 P] v_{0} / h-P \omega^{*}\right) \boldsymbol{e}_{y} \\
\boldsymbol{t}_{y}=-\mu_{\mathrm{eff}}\left([1-2 P] v_{0} / h-P \omega^{*}\right) \boldsymbol{e}_{y}\end{array}$ & $\begin{array}{l}\mathbf{0} \\
\mathbf{0}\end{array}$ \\
\hline $\begin{array}{l}\text { front } \\
\text { back }\end{array}$ & $\begin{array}{l}+\boldsymbol{e}_{z} \\
-\boldsymbol{e}_{z}\end{array}$ & $\begin{array}{l}\mathbf{0} \\
\mathbf{0}\end{array}$ & $\begin{array}{l}\boldsymbol{\mu}_{y}=(\beta-\gamma)\left(\omega^{\mathrm{d}} / h\right) \boldsymbol{e}_{y} \\
\boldsymbol{\mu}_{y}=-(\beta-\gamma)\left(\omega^{\mathrm{d}} / h\right) \boldsymbol{e}_{y}\end{array}$ \\
\hline
\end{tabular}

Table 1. Force and moment tractions on the flanks of the free body without the (constant) pressure contribution.

We now calculate all forces and moments acting on the free body. From (5), (6), and (15) the force and moment tractions, $\boldsymbol{n} \cdot \boldsymbol{\sigma}$ and $\boldsymbol{n} \cdot \boldsymbol{\mu}$, respectively, on the six flanks of the free body rectangular parallelepiped can be obtained. They are indicated in Figure 1 by means of arrows and double arrows pointing in the corresponding directions. Note that the balance of linear momentum implies $p=$ $p(x)$ for the pressure. In the pure Couette flow no pressure gradient in $\boldsymbol{e}_{x}$-direction is applied and hence we conclude that the pressure is a constant. Therefore, we do not mention the pressure in our considerations of the free body diagram since it cancels out in the equilibrium of forces and moments.

A few remarks are in order. Whilst the force tractions on the top and bottom plates are constant, the force tractions of the right and left sides are not. They depend on height $y$ and are given by

$$
\boldsymbol{n} \cdot \boldsymbol{\sigma}(y)= \pm\left[(\mu-\tau) \frac{\mathrm{d} v}{\mathrm{~d} y}-2 \tau \omega\right] \boldsymbol{e}_{y} .
$$

As customary in technical mechanics we replace them by statically equivalent averages located at the center of mass, i.e., at height $y=h / 2$, given by

$$
\boldsymbol{t}_{y}=\frac{1}{h} \int_{0}^{h} \boldsymbol{n} \cdot \boldsymbol{\sigma}(y) \mathrm{d} y,
$$

and this result is shown in Table 1 and in the figure. A similar remark holds for the moment tractions acting on the front and back surfaces:

$$
\boldsymbol{n} \cdot \boldsymbol{\mu}(y)= \pm(\beta-\gamma) \frac{\mathrm{d} \omega}{\mathrm{d} y} \boldsymbol{e}_{y}, \quad \boldsymbol{\mu}_{y}=\frac{1}{h} \int_{0}^{h} \boldsymbol{n} \cdot \boldsymbol{\mu}(y) \mathrm{d} y .
$$


Furthermore, note that by looking at the free body diagram the equilibrium of forces is immediately obvious, whereas equilibrium of moments is evident only for the momentum balance in $\boldsymbol{e}_{y}$ - but not in $\boldsymbol{e}_{z}$-direction. Here we must write ( $d$ being the depth of the channel)

$$
\begin{aligned}
\sum M_{z}^{(\mathcal{O})}= & \left(\mu_{z, \mathrm{t}}+\mu_{z, \mathrm{~b}}\right) \ell d+2 t_{y} h d \frac{\ell}{2}-2 t_{x} \ell d \frac{h}{2} \\
= & 2 \mu_{\mathrm{eff}} P\left(v_{0}+h \omega^{*}\right) \ell d+\mu_{\mathrm{eff}}\left([1-2 P] \frac{v_{0}}{h}-P \omega^{*}\right) h d \ell \\
& -\mu_{\mathrm{eff}}\left(\frac{v_{0}}{h}+P \omega^{*}\right) \ell d h \equiv 0 .
\end{aligned}
$$

Moreover, from Table 1 we conclude that in order to keep the lower and upper plates at constant translational velocities 0 and $v_{0}$, and to ensure constant angular velocities $\omega_{\mathrm{t}}$ and $\omega_{\mathrm{b}}$, we must apply constant horizontal shear-like force tractions $t_{x}$ and two constant out-of-plane moment tractions $\mu_{z, \mathrm{t}}$ and $\mu_{z, \mathrm{~b}}$ as

$$
\begin{aligned}
t_{x} & =\mu_{\mathrm{eff}} \frac{v_{0}}{h}+\mu_{\mathrm{eff}} P \omega^{*}, \quad \mu_{z, \mathrm{t}}=\mu_{\mathrm{eff}} P\left[v_{0}+h \omega^{*}\right]+\frac{\mu N^{2}}{P L^{2}} h \omega^{\mathrm{d}}, \\
\mu_{z, \mathrm{~b}} & =\mu_{\mathrm{eff}} P\left[v_{0}+h \omega^{*}\right]-\frac{\mu N^{2}}{P L^{2}} h \omega^{\mathrm{d}},
\end{aligned}
$$

which can be inverted to represent the boundary conditions from (11)

$$
\begin{aligned}
v_{0} & =\frac{2 h t_{x}-\left(\mu_{z, \mathrm{t}}+\mu_{z, \mathrm{~b}}\right)}{2 \mu}, \\
\omega_{\mathrm{t}} & =\frac{\left(L^{2} P^{2} / N^{2}\right)\left(\mu_{z, \mathrm{~b}}-\mu_{z, \mathrm{t}}\right)-\left(\mu_{z, \mathrm{~b}}+\mu_{z, \mathrm{t}}\right)+2 h P t_{x}}{4 h P \mu}, \\
\omega_{\mathrm{b}} & =\frac{\left(L^{2} P^{2} / N^{2}\right)\left(\mu_{z, \mathrm{~b}}-\mu_{z, \mathrm{t}}\right)+\left(\mu_{z, \mathrm{~b}}+\mu_{z, \mathrm{t}}\right)-2 h P t_{x}}{4 h P \mu} .
\end{aligned}
$$

This gives us the possibility to employ "force-controlled" boundary conditions, $t_{x}, \mu_{z, \mathrm{t}}$, and $\mu_{z, \mathrm{~b}}$, instead of "velocity-controlled" ones, namely $v_{0}, \omega_{\mathrm{t}}$, and $\omega_{\mathrm{b}}$. The "forces" as well as "moments" depend on both the prescribed velocity and the prescribed spin. All of this is compatible with the conviction of Eulerian mechanists according to which the balances of momentum and of total moment of momentum are fully independent and so are the boundary conditions, may they be translational and angular velocities or forces and moments per unit area. The two latter pairs are dual to each other so to speak and, with a grain of salt, we may want to refer to them also as Dirichlet and Neumann conditions, respectively [Łukaszewicz 1999, p. 25 and p. 31]. 
Equation (23) also allows us to adapt the solutions shown in (15) to the forcecontrolled situation easily as

$$
\begin{aligned}
& \frac{v(\bar{y})}{h t_{x} / \mu}=\bar{y}-\frac{\left(\mu_{z, \mathrm{t}}+\mu_{z, \mathrm{~b}}\right)}{4 h t_{x}}(\left.1+\frac{\sinh (N L[2 \bar{y}-1])}{\sinh (N L)}\right) \\
&+\frac{\left(\mu_{z, \mathrm{t}}-\mu_{z, \mathrm{~b}}\right)}{4 h t_{x}}\left(1-\frac{\cosh (N L[2 \bar{y}-1])}{\cosh (N L)}\right), \\
& \frac{\omega(\bar{y})}{t_{x} / \mu}=-\frac{1}{2}+\frac{P L^{2}}{4 N^{2}} \frac{\left(\mu_{z, \mathrm{t}}-\mu_{z, \mathrm{~b}}\right)}{h t_{x}} \frac{\sinh (N L[2 \bar{y}-1])}{\sinh (N L)} \\
&+\frac{1}{4 P} \frac{\left(\mu_{z, \mathrm{t}}+\mu_{z, \mathrm{~b}}\right)}{h t_{x}} \frac{\cosh (N L[2 \bar{y}-1])}{\cosh (N L)},
\end{aligned}
$$

and in the special case of antagonistic spin boundary conditions, i.e., $\omega^{*}=0$, as

$$
\begin{aligned}
& \frac{v(\bar{y})}{h t_{x} / \mu}=\bar{y}-P\left[1+\frac{\sinh (2 N L[\bar{y}-1])}{\sinh (2 N L)}\right]+\frac{\xi}{2}\left[1-\frac{\cosh (N L[2 \bar{y}-1])}{\cosh (N L)}\right], \quad \text { (25) } \\
& \frac{\omega(\bar{y})}{t_{x} / \mu}=-\frac{1}{2}\left[1-\frac{\cosh (2 N L[\bar{y}-1])}{\cosh ^{2}(N L)}\right]+\frac{\xi}{2} \frac{L}{N} \frac{\sinh (N L[2 \bar{y}-1])}{\cosh (N L)}, \quad \xi=\frac{\mu_{z, \mathrm{t}}}{h t_{x}} .
\end{aligned}
$$

They allow us to study what happens if we keep the force or moment tractions, $t_{x}, \mu_{z, \mathrm{t}}$, and $\mu_{z, \mathrm{~b}}$, at a constant level: we expect that the velocities of the upper plate go down if the effective viscosity increases, i.e., if we depart from the case of classical Navier-Stokes. This we shall discuss in the next section.

In the special case $\omega_{\mathrm{t}}=-\omega_{\mathrm{b}}=-\omega_{0}$, and subsequently $\omega^{*}=0$, it is curious that the "force", $t_{x}$ in (22), depends only on $v_{0}$ whereas the "moment", $\mu_{z, t}$, depends on both $v_{0}$ and $\omega_{0}$. In order to achieve a certain top plate velocity whilst the spin on the boundaries is of the same magnitude but of different sign, a traction $t_{x}$ that is similar to the ordinary Navier-Stokes fluid case needs to be applied. The difference from the classical solution lies in the effective shear viscosity which contains many of the new viscosities. For simplicity we will discuss in what follows only the special case of "opposite" spin boundary conditions (as in [Řiha 1975] or [Singh 1982]) so that $\omega^{*}$ vanishes in all further considerations.

A singular case arises if we require "no-slip" boundary conditions for the angular velocity. Then $\omega_{0}=0$ and obviously $\mu_{z, \mathrm{t}}=h P t_{x}$, so that the force seems to dictate the behavior of the moment, which is an unpleasant thought from the viewpoint of Eulerian mechanists. We will get back to this issue in the discussion section of this paper.

We now turn to the task of computing the temperature increase due to stationary viscous flow. The following stationary heat conduction equation results from the energy balance (4) and attains the following form if the ansatz (9), together with 
$T=T(y)$, is used:

$$
0=\kappa \frac{\mathrm{d}^{2} T}{\mathrm{~d} y^{2}}+(\mu+\tau)\left(\frac{\mathrm{d} v}{\mathrm{~d} y}\right)^{2}+4 \tau \omega^{2}+4 \tau \omega \frac{\mathrm{d} v}{\mathrm{~d} y}+(\beta+\gamma)\left(\frac{\mathrm{d} \omega}{\mathrm{d} y}\right)^{2} .
$$

The integration can be performed in closed form. For the boundary conditions we assume that two reservoirs of constant temperature $T_{\text {res }}$ are attached to the upper and lower plates:

$$
\begin{aligned}
\frac{T(\bar{y})-T_{\text {res }}}{T_{\text {ref }}}=\frac{a}{2}\{\bar{y}(1-\bar{y})- & \frac{1}{L^{2}}\left(1-\frac{\cosh (N L[2 \bar{y}-1])}{\cosh (N L)}\right) \\
& \left.-\frac{1}{8 L^{2}} \frac{\cosh (2 N L[2 \bar{y}-1])-\cosh (2 N L)}{\cosh ^{2}(N L)}\right\} \\
& -\frac{b}{4 L^{2}}\left(2 \bar{y}-1-\frac{\sinh (N L[2 \bar{y}-1])}{\sinh (N L)}\right) \\
& -\frac{c}{4 L^{2}} \frac{\cosh (2 N L[2 \bar{y}-1])-\cosh (2 N L)}{\sinh ^{2}(N L)}
\end{aligned}
$$

with the "dimensionless" temperatures

$$
a=\frac{\mu v_{0}^{2}}{\kappa T_{\mathrm{ref}}}\left(\frac{\mu_{\mathrm{eff}}}{\mu}\right)^{2}, \quad b=\frac{\mu v_{0} h \omega_{0}}{\kappa T_{\mathrm{ref}}} \frac{\mu_{\mathrm{eff}}}{\mu}, \quad c=\frac{\mu\left(h \omega_{0}\right)^{2}}{\kappa T_{\mathrm{ref}}} .
$$

The first term in the curly brackets is the classic parabolic departure from a constant temperature, symmetrically centered about the middle of the channel. Obviously the viscosities incorporated in the coupling number $N$ and in the length scale parameter $L$ have an effect on the temperature variation. The expressions in the $a$-term are related to pure translational velocity control of the top plate, the $c$-term includes the influence of pure angular velocity control, and the $b$-term represents the interaction between both.

With the relations shown in (23) it is straightforward to rewrite the temperature in terms of force boundary conditions:

$$
\begin{aligned}
\frac{T(\bar{y})-T_{\text {res }}}{T_{\text {ref }} a / 2}=\bar{y}(1- & \bar{y})-\frac{1}{L^{2}}\left(1-\frac{\cosh (N L[2 \bar{y}-1])}{\cosh (N L)}\right) \\
& -\frac{L^{2} P^{2}}{8 N^{2}}\left(P^{2}-2 P \xi+\xi^{2}\right) \frac{\cosh (2 N L[2 \bar{y}-1])-\cosh (2 N L)}{\sinh ^{2}(N L)} \\
& -\frac{P}{4 N^{2}}(P-\xi)\left(2 \bar{y}-1-\frac{\sinh (N L[2 \bar{y}-1])}{\sinh (N L)}\right) \\
& -\frac{1}{8 L^{2}} \frac{\cosh (2 N L[2 \bar{y}-1])-\cosh (2 N L)}{\cosh ^{2}(N L)}
\end{aligned}
$$




\section{Exploitation of the analytical results}

Consider first the velocity-controlled profiles described by (15). Let us initially assume that $\omega_{0}=0$ and that $N^{2}=0.9$, i.e., strong coupling. Then an S-shaped form of the velocity profile becomes apparent: Figure 2, left. Note that for small values of $L$ as well as for large values the velocity profiles almost coincide with the classical Navier-Stokes while for intermediate values of $L$ the $S$-shape is more pronounced.

In [Ivanova and Vilchevskaya 2016] the particle aspect on a mesoscopic scale was connected to the continuum point of view of a micropolar medium. If we follow the authors' line of reasoning we may argue intuitively and say that for the case when $L$ is very small, i.e., the length of the particles on the mesoscale becomes comparable or greater than the (macroscopic) plate distance, there is no "space for rotation", and the angular velocity vanishes (blue line). From (17) it is clear that the angular velocity can only arise from applied angular velocities at the boundary and is not influenced by the linear velocity boundary conditions in the limit case $L \rightarrow 0$. Furthermore note that in this case the linear velocity is given by the classical Navier-Stokes solution if no-spin boundary conditions are employed; see (17) and Figure 2, left. After normalization with the plate velocity the effect of intermediate length scales on the angular velocity, Figure 2, right, becomes visible: with increasing $L=h / l$, the length of the particles on the mesoscale $l$ compared to the plate distance $h$ allows for more rotation and thus greater magnitudes of the angular velocity occur until the size effect disappears for very large $L$. Observe that for $L=2$ the velocity gradient in the middle of the channel is greater than for an ordinary Navier-Stokes fluid. Furthermore, note that in this case the characteristic
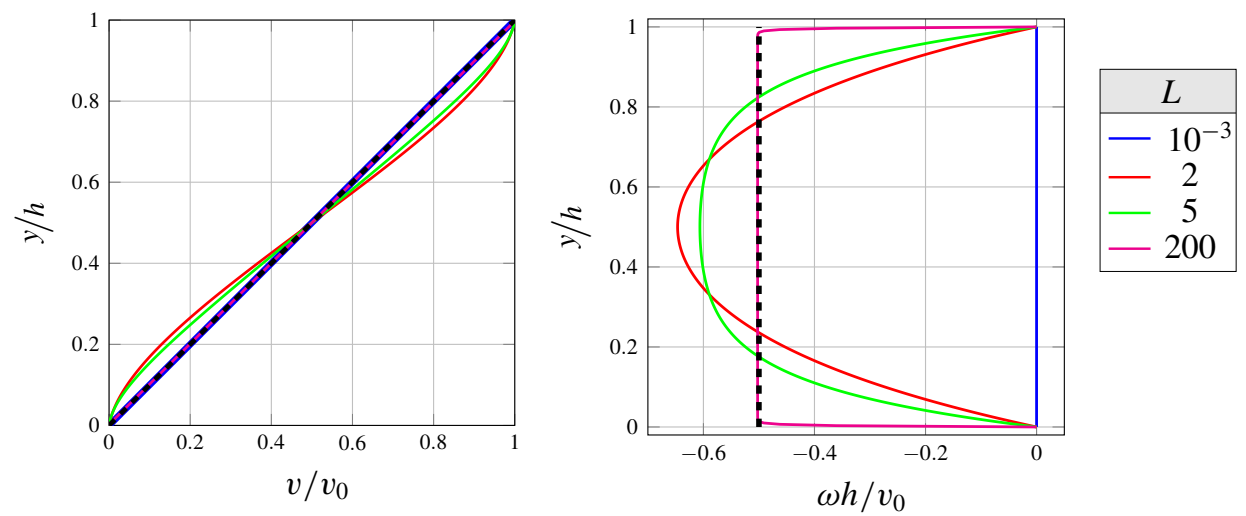

Figure 2. Linear and angular velocity profiles for Couette flow according to the Eringen theory for the velocity-controlled case with no-spin boundary conditions, $\omega_{0}=0$. 

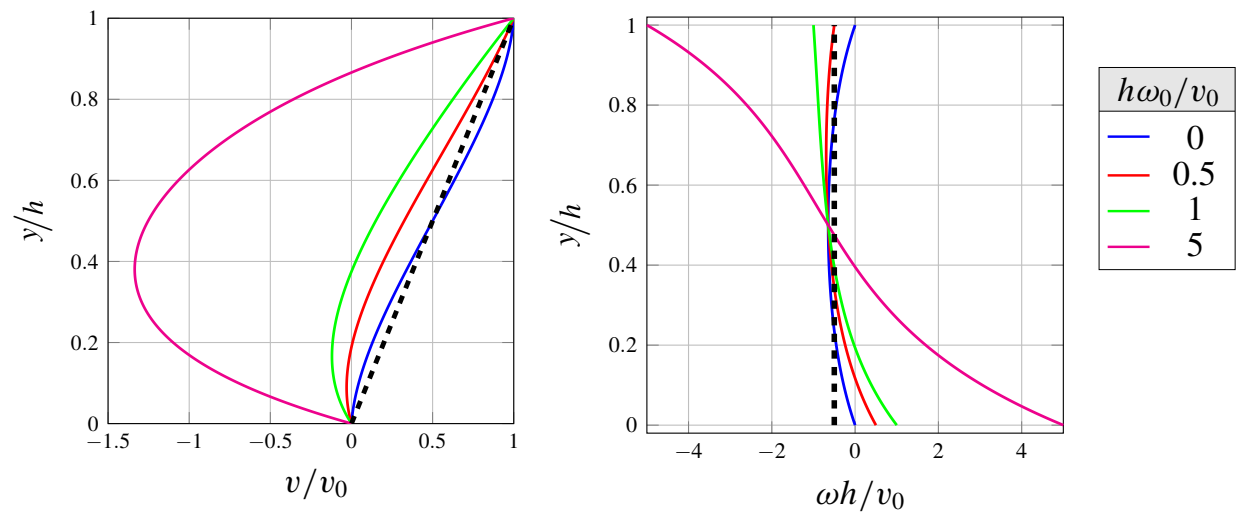

Figure 3. Linear and angular velocity profiles for Couette flow according to the Eringen theory for the velocity-controlled case.

length scale is half of the plate distance. Due to the higher shear rate and the length scale that fits twice into the channel, the fluid particles can rotate more easily and subsequently a greater magnitude of the angular velocity occurs, as can be seen in Figure 2, right. The dashed lines corresponds to a Navier-Stokes fluid, where in Figure 2, right, the vorticity was used because no angular velocity is available for ordinary fluids. ${ }^{3}$

In summary we may say that the plots for the linear velocity have been shown before in the literature [Cowin 1974, p. 323]. However, to the best knowledge of the authors this is surprisingly not so for the angular velocity plot.

In the previous figure we presented solutions for the no-spin boundary condition, i.e., $\omega_{0}=0$. From this case the solutions for $L=2$ are of particular interest and we shall now proceed to employ spin boundary conditions different from zero and keep $L=2$ fixed. In Figure 3 the linear and angular velocity profiles are depicted for different values of the factor $h \omega_{0} / v_{0}$. For sufficiently large values of this factor a significant backflow behavior is observable and the S-shaped velocity profiles turn into more and more wavy curves (Figure 3, left). The angular velocity is no longer an even but an odd function since the boundary conditions at the upper and lower plate only coincide in magnitude but have different signs. As we shall see immediately this behavior changes when we switch to the force-controlled case.

For the case of force control the velocity, normalized by $h t_{x} / \mu$, is illustrated in Figure 4, left. A purely force-controlled case was assumed, i.e., there is no moment per unit area acting on the upper plate, $\mu_{z, \mathrm{t}}=0$, and $N^{2}=0.9$, as before. As to be expected we obtain the bisectrix of ordinary Navier-Stokes flow if the internal length scale is negligible (large values of $L$ ). Intermediate length scales lead to

\footnotetext{
${ }^{3}$ The same mode of presentation will be used in the following plots.
} 

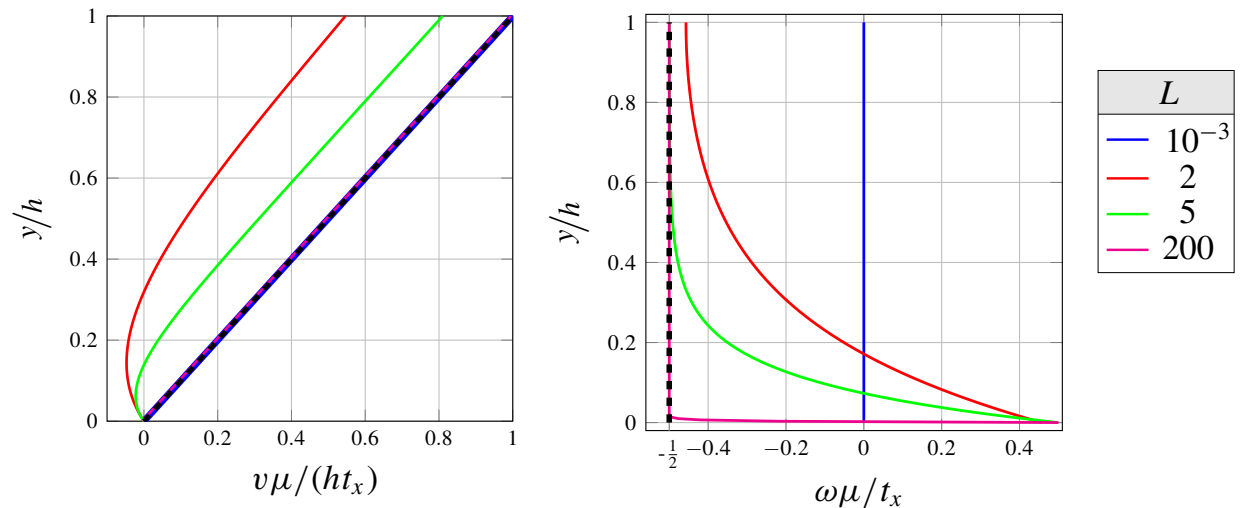

Figure 4. Linear and angular velocity profiles for Couette flow according to the Eringen theory for the force-controlled case with $\mu_{z, \mathrm{t}}=0$.

S-shaped curves showing, most interestingly, backflow behavior. This has been reported recently for nonstationary Couette flow [Magyari et al. 2010], as well as in molecular dynamics simulations of micropolar fluid flow [Haghighi and Asl 2015]. Very small values of $L$ lead again to a straight line but with a much smaller velocity at the top plate which, for equal $t_{x}$, results because of the effectively higher shear viscosity. Also note that for a prescribed force the velocity of the upper plate goes down if the effective viscosity increases, i.e., if we depart from the case of classical Navier-Stokes. This is also intuitively plausible.

It should be mentioned that a similar case of normalization of the velocity is also reported in [Cowin 1974, p. 326], where the case of vanishing angular velocities at the plate boundaries (no slip of the $\omega$-field) was exclusively considered. However, there is a subtle difference from our case if we specialize to $\omega_{0}=0$. According to $(23)_{2}$ a vanishing angular velocity on the top plate corresponds to a nonvanishing moment per unit area, $\mu_{z, \mathrm{t}}=h t_{x} P$. Moment and force are not independent in this case, and one ends up with the relations

$$
\begin{aligned}
\frac{v(\bar{y})}{h t_{x} / \mu} & =\bar{y}-\frac{P}{2}\left(1+\frac{\sinh (N L[2 \bar{y}-1])}{\sinh (N L)}\right), \\
\frac{\omega(\bar{y})}{t_{x} / \mu} & =-\frac{1}{2}+\frac{\cosh (N L[2 \bar{y}-1])}{2 \cosh (N L)} .
\end{aligned}
$$

Thus, we cannot say that Cowin [1974] considers a proper force-controlled case, and in fact he never says he does. Be this as it may, (30) does not show any backflow behavior.

The angular velocity also depends considerably on height, at least for values of $L$ in between the upper and lower bounds: Figure 4, right. If the length scale of 

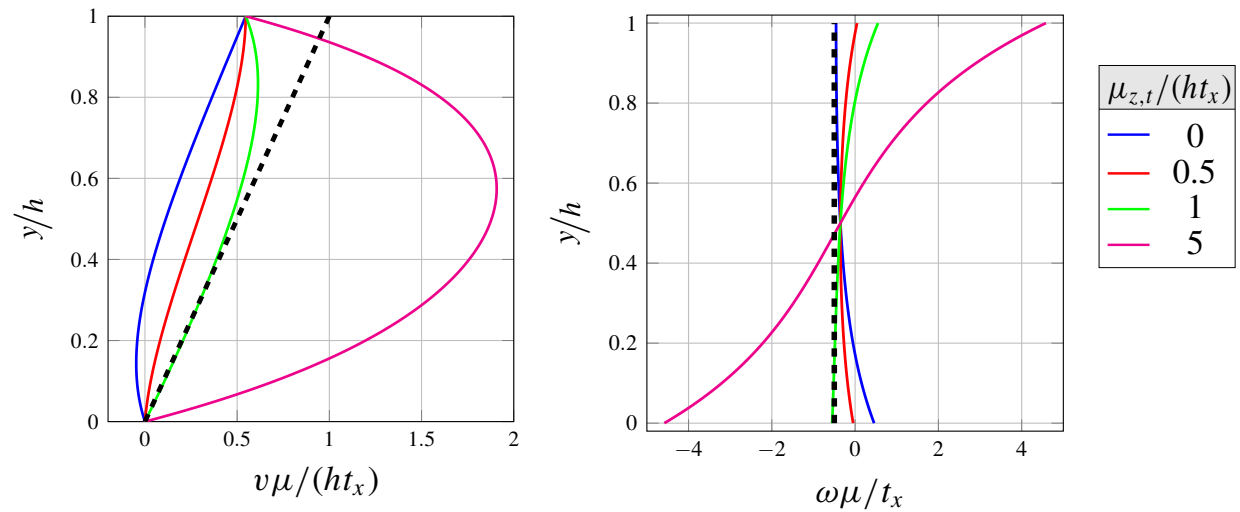

Figure 5. Linear and angular velocity profiles for Couette flow according to the Eringen theory for the force-controlled case.

the micropolar fluid is small compared to the distance of the plates (large values of $L$ ), the angular velocity is completely represented by the vorticity, which is constant: $\omega /\left(t_{x} / h\right)=-\frac{1}{2}$ (magenta curve), as in the case of Navier-Stokes flow (black dashed curve).

The analogue to Figure 3 is shown in Figure 5 (for $L=2$ ). In Figure 5, left, the velocity profiles normalized by the horizontal traction at the upper plate show backflow behavior as well but tend to be more pronounced in the positive $\boldsymbol{e}_{x}$-direction for higher values of the ratio of applied tractions $\mu_{z, \mathrm{t}} /\left(h t_{x}\right)$. In addition, the angular velocity profiles are essentially the same as in the velocity-controlled case but with a different sign so that the angular velocity at the upper plate is now positive.

As far as the temperature profiles of (27) are concerned we first focus on the case where $T_{\text {ref }}=\mu v_{0}^{2} / \kappa$ (in accordance with the normalization used in the classical Navier-Stokes case; see, e.g., [Müller 2014, p. 327]) and $a=\left(\mu_{\mathrm{eff}} / \mu\right)^{2}=$ $(1-P)^{-2}, b=c=0$, i.e., the case of pure translational velocity control. This is shown in Figure 6, left, by using the same parameters as for the plots of Figure 4. Obviously the temperature change in micropolar fluid flow is more pronounced than for a classical Navier-Stokes fluid (dashed line). This is to be expected because of the additional viscosity terms, in particular because of the factor $(1-P)^{-2}$.

Figure 6 , right, concentrates on the case $a=b=0$, i.e., the case of pure angular velocity control. It is now tempting to analyze the situation for $c=1$. However, note that in the limit case $L \rightarrow 0$ (while keeping $\beta+\gamma=$ const.) one has to take into account that the parameter $c$ will not remain constant because

$$
c=\frac{\mu\left(h \omega_{0}\right)^{2}}{\kappa T_{\mathrm{ref}}}=\frac{L^{2}(\beta+\gamma) \omega_{0}^{2}}{\kappa T_{\mathrm{ref}}}
$$



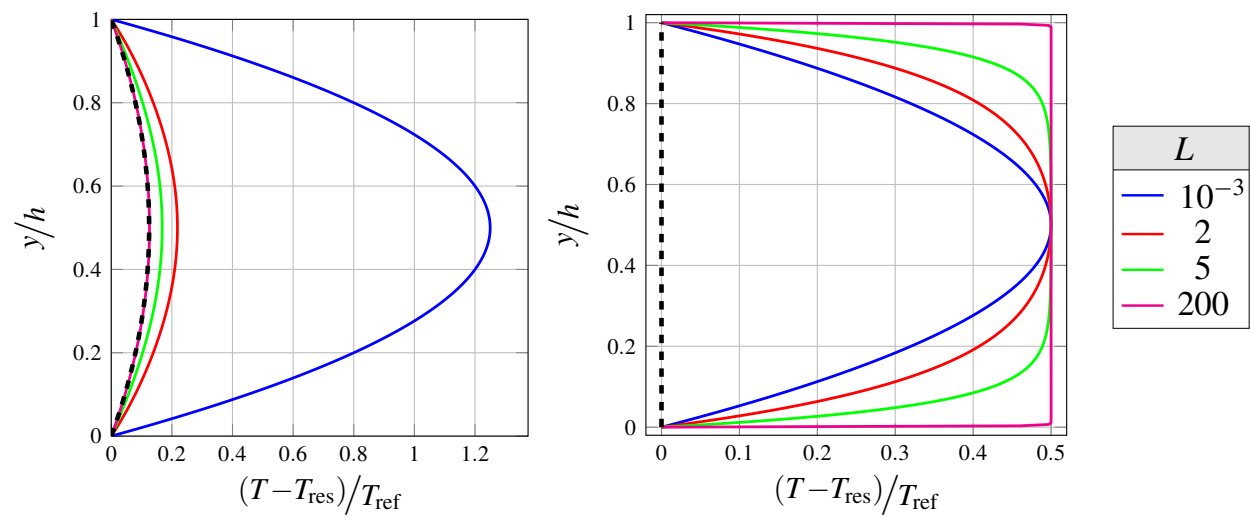

Figure 6. Temperature profiles for Couette flow according to Eringen's micropolar theory.

strongly depends on $L$. Choosing the reference temperature conveniently as $T_{\text {ref }}=$ $(\beta+\gamma) \omega_{0}^{2} / \kappa$ we must put $c=L^{2}$, which balances the otherwise singular term in (27) for very small values of $L$, leading to parabolic profiles. On the other hand, very large values of $L$ imply that the internal length scale is negligible when compared to the plate distance so that the temperature becomes independent from the position between the plates. This leads to a homogeneous temperature profile. Note that this situation does not correspond to an ordinary fluid because there is no angular velocity available in the classical Navier-Stokes case, $\omega \equiv 0$; see Figure 6, right.

\section{Literature review and discussion}

Since the advent of Eringen's theory of micropolar fluids simple flow problems allowing for analytical solutions have continuously been in the focus of interest. Poiseuille flow is most predominantly discussed. In fact, Eringen [1966] gives corresponding solutions for translational and angular velocity in circular pipes, which are later repeated in his textbooks, e.g., [Eringen 2001, §9.7]. The temperature rise due to viscous friction for Poiseuille flow between plates is discussed in [Kazakia and Ariman 1971; Řiha 1975]. A first discussion of the velocity profiles of plane-parallel micropolar Couette flow can be found in [Ariman and Cakmak 1968; Pennington and Cowin 2000; Cowin 1974]. However, these authors constrain themselves to the case of vanishing angular velocity at the plates, $\omega_{0}=0$. Couette flow between rotating cylinders was studied in [Verma and Sehgal 1968], where it was also assumed that the rotational velocities vanish at the cylinder walls.

A discussion of the temperature rise in plane-parallel Couette flow is presented in [Singh 1982]. Although Singh applies his solution to the concrete example of blood flow the general presentation is essentially a mathematical exercise allowing for 
the greatest freedom in the choice of boundary conditions. For example, the effect of microtemperatures is included just like different choices of the top and bottom reservoir temperatures. In a nutshell the final result contains so many abbreviations that it becomes impossible to discern the impact of the various physical parameters. It is for this reason that we have constrained ourselves in this presentation to the most essential ones.

Due to the advent of micro- and nanotechnology the solution to elementary flow problems based on micropolar theory complemented by molecular dynamics simulations is more recently in the focus of research again. Examples are given by [Kucaba-Pietal 2004; Kucaba-Pietal et al. 2009].

Chen et al. [2012] and Ashmawy [2012] present an analysis of transient Couette flow, based on a numerical and a Laplace transform solution, respectively. The angular velocity at the boundaries was once more assumed to be zero.

Hence, it is now high time to talk about how boundary conditions for micropolar media are usually introduced in the literature and, what is even more important, if and how they can be realized experimentally.

It is obvious from the citations we presented so far that it is very popular to postulate so-called "no-slip" conditions or, in other words, that the tangential (translational) velocity of the micropolar fluid is equal to that of the wall and its angular velocity vanishes. However, recall that even in the simpler case of a Navier-Stokes fluid a no-slip condition is by no means as straightforward to realize as we may want to believe at first glance. We simply got so used to it that we rarely question it. However, the scientists who initiated this notion thought differently. From the citations in the nicely written overview on the history of the no-slip condition [Day 1990], we learn that Stokes based his conviction of the applicability of the noslip conditions on certain experiments, in particular one performed by Coulomb, according to which the resistance of water flow against a wall is independent of the surface characteristics (also see [Müller 2007, p. 241]). Hence, it is due to internal friction of the fluid alone, which is consistent with the assumption that the water simply sticks to the wall. This statement was questioned by Maxwell [Brenner 2011] and replaced by the possibility of slip in the case of dilute gases, a phenomenon which becomes ever more important to be taken into account in today's microfluidic systems [Lauga et al. 2007]. Practically speaking, the amount of slip encountered in a particular experimental setup may be controlled (to a certain degree) by polymer or surfactant absorption or other chemical modification of the walls.

Moving now closer to the boundary conditions relevant for micropolar fluids, it should be mentioned that slip of the tangential velocity at a wall was recently considered in order to study the Couette flow of couple stress fluids in [Silber et al. 2007; Alizadeh et al. 2011; Devakar et al. 2014]. The model of a couple stress 
fluid becomes relevant if surface effects occur [Mindlin 1965]. For example, when we add additives to a fluid, the forces present in the fluid oppose the forces of the additives. This opposition leads to a couple force, and hence, a couple stress is induced in the fluid. We may say that this is also an example of materials that require a generalized continuum theory beyond the ordinary Boltzmann-Cauchy approach.

General properties of a couple stress theory for solids were analyzed in [Hadjesfandiari and Dargush 2011]. On the basis of the principle of virtual work and without the use of special constitutive relations the authors showed, e.g., that the moment traction vector is tangent to the surface. However, they restrict their presentation to the equilibrium case, i.e., their results lack generality. Although a couple stress theory for solids has little to do with the theory presented in this work, note that in the case of Couette flow the moment tractions are indeed tangential to the surfaces as can be seen in Table 1 .

Another generalized continuum which, in contrast to couple stress fluids, necessitates us to take the aspect of internal rotational degrees of freedom into account, are nematic liquid crystals. In order to describe their flow behavior the theory proposed by Ericksen and Leslie is frequently applied [Steward 2004]. There are certain differences when compared to the Eringen approach, which is favored in this paper. The microinertia tensor is replaced by the so-called director, often represented by an inextensible one-dimensional digit. It is the continuum representation of the macromolecules liquid crystals are made of. Note that the macromolecules are usually polarized, which is important for realizing the boundary conditions, as we shall discuss shortly. Other "new" quantities in the Ericksen-Leslie theory are the director stress tensor, which replaces the couple stress tensor, and the director production density, which occurs, most importantly, because the spin balance is replaced by what is known as the balance of director force.

In the Ericksen-Leslie theory boundary conditions for the angle of orientation of the director at a wall are required. Two main types of this so-called "strong anchoring" are distinguished [Steward 2004, p. 47], namely homeotropic and homogeneous alignment, where the digits are oriented perpendicular and parallel to the wall, respectively. Note that such conditions are the equivalent of prescribing a vanishing angular velocity at the wall in the case of Eringen's theory of micropolar fluids. But how is this mathematical requirement realized in practice? An answer can be found in [de Gennes and Prost 1995, p. 109]: the surfaces of the walls must be prepared accordingly by "rubbing" thus inducing a certain electric "polarization".

Finally, let us review the situation in the case of micropolar fluids. An excellent summary of the situation, at least from a mathematical point of view, can be found in [Łukaszewicz 1999, p. 30]. First, the author discusses velocity-type boundary 
conditions of the type shown in (11). Specifically he writes

$$
v=v_{\mathrm{b}}, \quad \omega=\omega_{\mathrm{b}},
$$

where it is explicitly mentioned that $\boldsymbol{v}_{\mathrm{b}}$ is the (translational) velocity and $\omega_{\mathrm{b}}$ is the microrotation of points of the solid boundary $b$. The author also refers to $(32)_{1}$ as "physically clear (the viscous fluid sticks to the solid boundary)" and then states that "there is no general agreement as to the type of boundary condition one should set for the field of microrotation" $(32)_{2}$. Indeed, it was mentioned before, and the proper references have been given, that frequently such no-slip boundary conditions are prescribed in micropolar flow problems, i.e., the tangential translational velocity is that of the wall and the angular velocity is either zero or of a fixed value, also characteristic of the wall particles. However, it is usually not discussed how these can be realized physically. In this context recall our remarks and references from above on the no-slip condition for the translational velocity.

However, there are some alternative opinions to the no-slip condition as well. For example, in the early paper by Ahmadi [1976] it is suggested that

$$
\omega=-\frac{1}{2} \frac{\mathrm{d} v}{\mathrm{~d} y} \quad \text { at } y=0, h .
$$

In other words, the angular velocity equals the vorticity, where both are related to fluid and not to wall characteristics. In fact the author says, "In the neighborhood of a rigid boundary the effect of microstructure must be negligible since the suspended particle cannot get closer than their radius to boundary. In the case of blood flow it is observed that the red cells tend not to get very close to the boundary... Therefore, in the neighborhood of the boundary the only rotation is due to fluid shear and therefore, gyration vector must be equal to angular velocity." Clearly, this also means that angular velocity and translational velocity are coupled and no longer independent, which is in disagreement with the beliefs of Eulerian mechanics.

However, this condition was generalized in [Kirwan $1986 ;{ }^{4}$ Kolpashchikov et al. 1983] by modifying it to

$$
\omega_{\mathrm{b}}=\alpha \boldsymbol{w}_{\mathrm{b}},
$$

where through the index " $b$ " reference is made to fluid properties at the boundary. In fact the latter authors say, "The quantity $v$ [i.e., $\omega$ ] in the fluid volume decreases with an increase of the effect of moment stresses between particles upon microrotation, tending to zero, at the absence of external fields. With a decrease of this influence the magnitude $v$ increases and tends to the limit $w=(1 / 2) \operatorname{rot} v$. Thus, a change of $v$ is restricted to limits from 0 to $w$... In the case of complete adhesion,

\footnotetext{
${ }^{4}$ Kirwan addressed the question of boundary conditions even earlier in a less general manner [Kirwan and Newman 1969a; 1969b].
} 
$v_{b}=0$, from where the lower limit of the parameter of boundary conditions $\alpha=0$ follows. With deterioration of the above interaction $v_{b}$ increases up to the value, also determined by moment stresses between the MPF [micropolar fluid] particles. When the moment stresses between the MPF particles and the boundary are sufficiently small, the magnitude $v_{b}$ is determined mainly by the moment stresses between the fluid particles themselves. In the problem under consideration this case is described by the condition"

$$
\left.\frac{\mathrm{d} \omega}{\mathrm{d} y}\right|_{\bar{y}=0 \text { or } 1}=0 .
$$

Continuing, "Equality $\alpha=1$ corresponds to the physical situation, where there are moment stresses and no difference between the angular velocity of particle proper rotation and that of the fluid volume microelement containing it, $w$. Thus, the range of variation of the parameter of boundary conditions is $0 \leq \alpha \leq 1$."

In short, (34) is an attempt to relate angular velocity and the rotation of the translational velocity. We may want to refer to $\alpha=1$ as the fully coupled case and vice versa. In a certain way this equation circumvents the assumption of the principle of independence of both types of velocities and, indeed, quoting Hogan and Henriksen [1989], "No compelling theoretical argument or experimental evidence suggesting a correct value for $S$ [i.e., $\alpha$ ] has been found in the literature."

Nevertheless, if we make use of this boundary condition for $\omega$ the constants $c_{i}$ in (12) can be determined to read

$$
\begin{aligned}
c_{1} & =-\frac{v_{0} P(1-\alpha)}{2\left[(1-P)-\alpha\left(N^{2}-P\right)\right]}, \quad c_{2}=\frac{\left(v_{0} / h\right)\left(1-\alpha N^{2}\right)}{(1-P)-\alpha\left(N^{2}-P\right)}, \\
c_{3 / 4} & =\frac{\left(v_{0} / h\right)(1-\alpha)[1 \mp \tanh (N L)]}{4\left[(1-P)-\alpha\left(N^{2}-P\right)\right]},
\end{aligned}
$$

and the following solutions are obtained:

$$
\begin{aligned}
\frac{v}{v_{0}} & =\frac{\bar{y}\left(1-\alpha N^{2}\right)-\frac{1}{2} P(1-\alpha)(1+\sinh (N L[2 \bar{y}-1]) / \sinh (N L))}{(1-P)-\alpha\left(N^{2}-P\right)}, \\
\frac{\omega}{v_{0} / h} & =\frac{1}{2} \frac{\left(1-\alpha N^{2}\right)-(1-\alpha) \cosh (N L[2 \bar{y}-1]) / \cosh (N L)}{P(1-\alpha)+\left(\alpha N^{2}-1\right)} .
\end{aligned}
$$

In [Kolpashchikov et al. 1983] the question was asked which value for $\alpha$ leads to the condition (35). The answer depends on the boundary value problem under study. In that reference an answer was given for the case of plane-parallel plate flow due to a pressure gradient. Then $\alpha$ can assume arbitrary values between 0 and 1, depending on the choice of micropolar fluid constants $N$ and $L$. If we wish to obtain (35) for our boundary value problem (36)-(37) we must obviously choose $\alpha=1$, i.e., only the case of total coupling prevails. 
More recently an alternative to (33) has been proposed in [Chakraborty and Chakraborty 2008]:

$$
\omega(\bar{y}=1 \text { or } 0)=\left.\beta \frac{\mathrm{d} \omega}{\mathrm{d} y}\right|_{\bar{y}=1 \text { or } 0} .
$$

The coefficient $\beta$, which has the dimension [m], is interpreted as the microrotational slip length and $\left.\frac{\mathrm{d} \omega}{\mathrm{d} y}\right|_{\bar{y}=1 \text { or } 0}$ as (proportional to) the couple stress $\mu_{y z}$ at the walls. This idea has been quantified by molecular dynamics simulations in that reference and continues to be very popular in the most recent literature. The following quote from [Sheikholeslami et al. 2014], where the parameter $s$ is identical to $-\beta$, provides some physical insight into this ansatz: "The case $s=0$ represents concentrated particle flows in which microelements close to the wall are unable to rotate. Other interesting particular cases that have been considered in the literature include $s=0.5$ which represents weak concentrations and the vanishing of the antisymmetric part of the stress tensor and $s=1$ which represents turbulent flow."

Finally, note that molecular dynamics simulations indicate that choosing the noslip condition $\omega_{0}=0$ or a vanishing gradient of the angular velocity (38) seems to be linked to the surface roughness, or to quote Lopez et al. [2016], "The boundary conditions for microrotations... were chosen to be $\omega=0$ at the wall and inlet, and $\frac{\partial \omega}{\partial \eta}=0$ at the outlet and the far field boundary, where $\eta$ is the normal coordinate. The reason to prescribe $\omega=0$ at the inlet and at the wall is to avoid the effects of the wall roughness or perturbations from the free stream entering the boundary layer."

Indeed, such possibilities were anticipated by Łukaszewicz [1999, p. 31], when he talks about the possibility to prescribe forces and moments, as we did in Table 1 . Referring to [Aero et al. 1965] he proposes in a very general manner that at the wall we have

$$
\boldsymbol{n} \cdot \boldsymbol{\mu}=\boldsymbol{A} \cdot(\boldsymbol{\omega}-\boldsymbol{w})
$$

where " $\boldsymbol{A}$ is a matrix with numeric components". We may wish to refer to it as a coupling matrix instead according to the Eulerian credo that angular and translational velocities are independent.

The limit cases $\boldsymbol{A} \rightarrow \infty$ and $\boldsymbol{A} \rightarrow 0$ are treated by putting $\boldsymbol{\omega}=\boldsymbol{w}$, which expresses "a very strong interaction between the fluid particles and the solid boundary the microrotation reduces to the vorticity of points at the boundary caused by its movement". We decided to depict this situation by the cartoon shown in Figure 7. In particular, if the boundary does not move this condition reduces to $\boldsymbol{\omega}=\mathbf{0}$. Despite the nice cartoon shown in the figure, the question as to how to induce an $\omega \neq 0$ at a wall experimentally is unanswered until today and the same holds for the question of the transmission of a prescribed moment density. 


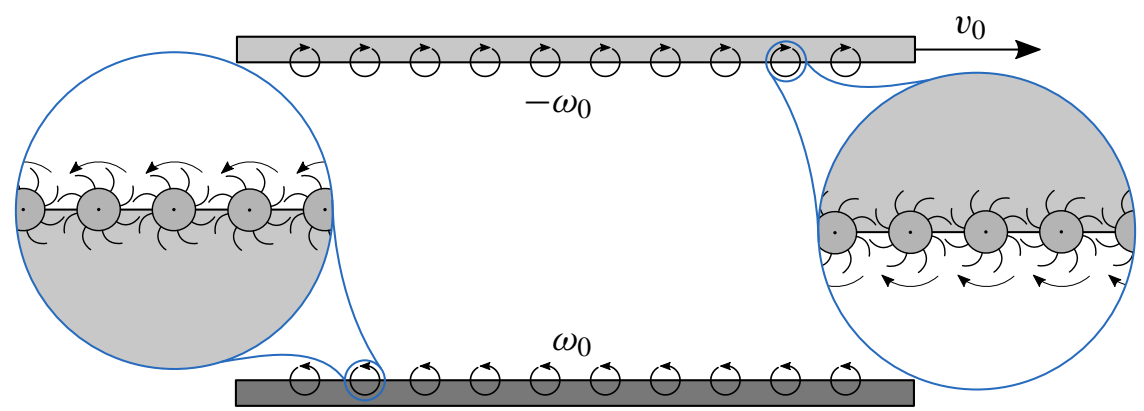

Figure 7. Model concept of imposing the angular velocity field via small paddlewheels.

More generally speaking we may also claim that the question of which boundary conditions to use in the case of theories beyond the Cauchy-Boltzmann continuum is still in need of finding a rational basis for their definition. Eventually the kinetic theory of gases might provide a clue: for the case of ideal gases it was demonstrated in [Barbera et al. 2004] that the missing boundary conditions for the heat flux in an extended thermodynamic theory can be found by minimization of the entropy of the system in question. Clearly, such an approach is much more difficult to realize if fluid matter is concerned.

\section{Conclusions and outlook}

In this paper we provided closed-form solutions of Couette velocity profiles based on Eringen's theory for micropolar fluids. In addition to that the temperature increase due to internal viscous friction was calculated analytically. We analyzed the effects of different boundary conditions and commented on suggestions for these from the literature. Possible corresponding experimental realizations were presented.

\section{Appendix A: Remarks on notation}

For convenience of the reader we present the relevant equations in Cartesian representation. The balances introduced in (1)-(4) read

$$
\begin{aligned}
& 0=\frac{\partial v_{i}}{\partial x_{i}}, \quad \rho_{0} \frac{\mathrm{d} v_{i}}{\mathrm{~d} t}=\frac{\partial \sigma_{j i}}{\partial x_{j}}, \quad \rho_{0} J_{0} \frac{\mathrm{d} \omega_{i}}{\mathrm{~d} t}=\frac{\partial \mu_{j i}}{\partial x_{j}}+\epsilon_{i j k} \sigma_{j k}, \\
& \rho_{0} \frac{\mathrm{d} u}{\mathrm{~d} t}=-\frac{\partial q_{i}}{\partial x_{i}}+\sigma_{i j}\left(\frac{\partial v_{j}}{\partial x_{i}}-\epsilon_{i j k} \omega_{k}\right)+\mu_{i j} \frac{\partial \omega_{j}}{\partial x_{i}},
\end{aligned}
$$


where $\epsilon_{i j k}$ is the Levi-Civita symbol. For the constitutive relations given in (5), (6), and (8) we have

$$
\begin{aligned}
\sigma_{i j} & =-p \delta_{i j}+\mu\left(\frac{\partial v_{i}}{\partial x_{j}}+\frac{\partial v_{j}}{\partial x_{i}}\right)+2 \tau \omega_{k} \epsilon_{k j i}-\tau\left(\frac{\partial v_{i}}{\partial x_{j}}-\frac{\partial v_{j}}{\partial x_{i}}\right), \\
\mu_{i j} & =\alpha \frac{\partial \omega_{k}}{\partial x_{k}} \delta_{i j}+\beta\left(\frac{\partial \omega_{i}}{\partial x_{j}}+\frac{\partial \omega_{j}}{\partial x_{i}}\right)-\gamma\left(\frac{\partial \omega_{i}}{\partial x_{j}}-\frac{\partial \omega_{j}}{\partial x_{i}}\right), \\
q_{i} & =-\kappa \frac{\partial T}{\partial x_{i}}+\lambda \frac{\partial \omega_{k}}{\partial x_{j}} \epsilon_{i j k},
\end{aligned}
$$

where $\delta_{i j}$ is the Kronecker symbol and $h_{i}=\omega_{i}-\frac{1}{2} \epsilon_{i j k} \frac{\partial v_{k}}{\partial x_{j}}$ was used. Finally, with the aid of the relations

$$
\begin{aligned}
& \nabla \cdot(\boldsymbol{h} \times \mathbf{I})=\frac{\partial}{\partial x_{i}} \boldsymbol{e}_{i} \cdot\left(h_{j} \boldsymbol{e}_{j} \times \boldsymbol{e}_{k} \otimes \boldsymbol{e}_{k}\right)=\frac{\partial h_{j}}{\partial x_{i}} \epsilon_{j k i} \boldsymbol{e}_{k}=\nabla \times \boldsymbol{h}, \\
& \boldsymbol{\sigma}_{\times}=2 \tau(\boldsymbol{h} \times \mathbf{I})_{\times}=2 \tau h_{i}\left(\boldsymbol{e}_{i} \times \boldsymbol{e}_{j}\right) \times \boldsymbol{e}_{j}=2 \tau h_{i} \epsilon_{i j k} \epsilon_{k j m} \boldsymbol{e}_{m}=-4 \tau \boldsymbol{h}, \\
& 2 \boldsymbol{w} \times \mathbf{I}=(\nabla \otimes \boldsymbol{v})^{\top}-\nabla \otimes \boldsymbol{v}, \\
& \nabla \times(\nabla \times \boldsymbol{v})=\nabla(\nabla \cdot \boldsymbol{v})-\Delta \boldsymbol{v}, \\
& \boldsymbol{e}_{i} \cdot \boldsymbol{e}_{j}=\delta_{i j}, \quad \boldsymbol{e}_{i} \times \boldsymbol{e}_{j}=\epsilon_{i j k} \boldsymbol{e}_{k}, \quad \epsilon_{i j k} \epsilon_{j k m}=2 \delta_{i m},
\end{aligned}
$$

where $\boldsymbol{e}_{i}$ are the base vectors, the balances of linear momentum and spin in (7) are obtained taking into account the incompressibility condition:

$$
\begin{aligned}
\rho_{0} \frac{\mathrm{d} v_{i}}{\mathrm{~d} t} & =-\frac{\partial p}{\partial x_{i}}+(\mu+\tau) \frac{\partial^{2} v_{i}}{\partial x_{k} \partial x_{k}}+2 \tau \frac{\partial \omega_{k}}{\partial x_{j}} \epsilon_{i j k}, \\
\rho_{0} J_{0} \frac{\mathrm{d} \omega_{i}}{\mathrm{~d} t} & =(\alpha+\beta-\gamma) \frac{\partial^{2} \omega_{j}}{\partial x_{i} \partial x_{j}}+(\beta+\gamma) \frac{\partial^{2} \omega_{i}}{\partial x_{k} \partial x_{k}}-4 \tau \omega_{i}+2 \tau \frac{\partial v_{k}}{\partial x_{j}} \epsilon_{i j k} .
\end{aligned}
$$

\section{Appendix B: Solution of the coupled system of partial differential equations}

In order to solve the boundary value problem shown in (10) the equations are recast into a system of first-order differential equations:

$$
\frac{\mathrm{d} \boldsymbol{u}}{\mathrm{d} y}=\boldsymbol{C} \cdot \boldsymbol{u} \quad \text { with } \quad \boldsymbol{u}=\left[\begin{array}{c}
v \\
v^{\prime} \\
\omega \\
\omega^{\prime}
\end{array}\right] \text { and } \boldsymbol{C}=\left[\begin{array}{cccc}
0 & 1 & 0 & 0 \\
0 & 0 & 0 & -\frac{2 \tau}{\mu+\tau} \\
0 & 0 & 0 & 1 \\
0 & \frac{2 \tau}{\beta+\gamma} & \frac{4 \tau}{\beta+\gamma} & 0
\end{array}\right] .
$$

By employing the standard exponential ansatz for $\boldsymbol{u}$ we obtain an eigenvalue problem

$$
\boldsymbol{u}=\exp (\lambda y) \boldsymbol{r} \quad \Longrightarrow \quad \lambda \boldsymbol{r}=\boldsymbol{C} \cdot \boldsymbol{r}
$$


If $\boldsymbol{C}$ has four linear independent eigenvectors $\boldsymbol{r}_{i}$, then the general solution to the system (44) is given by

$$
\boldsymbol{u}=\sum_{i=1}^{4} c_{i} \boldsymbol{u}_{i}=\sum_{i=1}^{4} c_{i} \exp \left(\lambda_{i} y\right) \boldsymbol{r}_{i},
$$

where $\lambda_{i}$ are the eigenvalues of $\boldsymbol{C}$ and $c_{i}$ are arbitrary constants. However, in our case $\boldsymbol{C}$ does not have four independent eigenvectors:

$$
\begin{aligned}
& \lambda_{1 / 2}=0, \quad \lambda_{3 / 4}= \pm \frac{2 N L}{h}, \boldsymbol{r}_{1}=\left[\begin{array}{l}
1 \\
0 \\
0 \\
0
\end{array}\right], \quad \boldsymbol{r}_{3 / 4}=\left[\begin{array}{c}
\mp N h / L \\
-2 N^{2} \\
1 \\
\pm 2 N L / h
\end{array}\right], \\
& \text { with } \quad N=\sqrt{\frac{\tau}{\mu+\tau}}, \quad L=\frac{h}{l}, \quad \text { and } \quad l=\sqrt{\frac{\beta+\gamma}{\mu} .}
\end{aligned}
$$

The (double) eigenvalue $\lambda_{1 / 2}$ is incomplete which means that only one linear independent eigenvector $\boldsymbol{r}_{1}$ can be found. Hence, an independent solution $\boldsymbol{u}_{2}$ needs to be constructed by setting [Walter 1998, p. 183]

$$
\boldsymbol{u}_{2}=\exp \left(\lambda_{1} y\right)\left(y \boldsymbol{r}_{1}+\boldsymbol{r}_{2}\right), \quad\left(\boldsymbol{C}-\lambda_{1} \mathbf{1}\right) \cdot \boldsymbol{r}_{2}=\boldsymbol{r}_{1} \quad \Longrightarrow \quad \boldsymbol{r}_{2}=\left[\begin{array}{llll}
0 & 1 & -\frac{1}{2} & 0
\end{array}\right]^{\top},
$$

where $\mathbf{1}$ is the unit matrix. Then, the first and third components of the linear combination of $\boldsymbol{u}_{i}$ are the wanted solutions.

\section{References}

[Aero et al. 1965] E. L. Aero, A. N. Bulygin, and E. V. Kuvshinskii, "Asymmetric hydromechanics", J. Appl. Math. Mech. 29:2 (1965), 333-346.

[Ahmadi 1976] G. Ahmadi, "Self-similar solution of imcompressible micropolar boundary layer flow over a semi-infinite plate”, Internat. J. Engrg. Sci. 14:7 (1976), 639-646.

[Alizadeh et al. 2011] M. Alizadeh, G. Silber, and A. Ghasemi Nejad, "A continuum mechanical gradient theory with an application to fully developed turbulent flows", J. Disper. Sci. Technol. 32:2 (2011), 185-192.

[Ariman and Cakmak 1968] T. Ariman and A. S. Cakmak, "Some basic viscous flows in micropolar fluids", Rheol. Acta 7:3 (1968), 236-242.

[Ashmawy 2012] E. A. Ashmawy, "Unsteady Couette flow of a micropolar fluid with slip", Meccanica 47:1 (2012), 85-94.

[Barbera et al. 2004] E. Barbera, I. Müller, D. Reitebuch, and N.-R. Zhao, "Determination of boundary conditions in extended thermodynamics via fluctuation theory", Contin. Mech. Thermodyn. 16:5 (2004), 411-425.

[Brenner 2011] H. Brenner, "Beyond the no-slip boundary condition", Phys. Rev. E 84:4 (2011), 046309. 
[Chakraborty and Chakraborty 2008] D. Chakraborty and S. Chakraborty, "Towards a generalization of hydrodynamic boundary conditions for flows of fluids with homogeneous internally rotating structures", Phys. Lett. A 372:33 (2008), 5462-5466.

[Chapman and Cowling 1952] S. Chapman and T. G. Cowling, The mathematical theory of nonuniform gases: an account of the kinetic theory of viscosity, thermal conduction and diffusion in gases, 2nd ed., Cambridge University, 1952.

[Chen et al. 2012] J. Chen, C. Liang, and J. D. Lee, "Numerical simulation for unsteady compressible micropolar fluid flow”, Comput. Fluids 66 (2012), 1-9.

[Condiff and Dahler 1964] D. W. Condiff and J. S. Dahler, "Fluid mechanical aspects of antisymmetric stress", Phys. Fluids 7 (1964), 842-854.

[Cowin 1974] S. C. Cowin, "The theory of polar fluids", Adv. Appl. Mech. 14 (1974), 279-347.

[Day 1990] M. A. Day, "The no-slip condition of fluid dynamics”, Erkenntnis 33:3 (1990), 285-296.

[Devakar et al. 2014] M. Devakar, D. Sreenivasu, and B. Shankar, "Analytical solutions of couple stress fluid flows with slip boundary conditions”, Alexandria Engrg. J. 53:3 (2014), 723-730.

[Eringen 1966] A. C. Eringen, "Theory of micropolar fluids”, J. Math. Mech. 16 (1966), 1-18.

[Eringen 1999] A. C. Eringen, Microcontinuum field theories, I: Foundations and solids, Springer, 1999.

[Eringen 2001] A. C. Eringen, Microcontinuum field theories, II: Fluent media, Springer, 2001.

[Gauthier and Jahsman 1975] R. D. Gauthier and W. E. Jahsman, "A quest for micropolar elastic constants", J. Appl. Mech. 42:2 (1975), 369-374.

[de Gennes and Prost 1995] P. G. de Gennes and J. Prost, The physics of liquid crystals, 2nd ed., Oxford University, 1995.

[Hadjesfandiari and Dargush 2011] A. R. Hadjesfandiari and G. F. Dargush, "Couple stress theory for solids", Int. J. Solids Struct. 48:18 (2011), 2496-2510.

[Haghighi and Asl 2015] A. R. Haghighi and M. S. Asl, "Mathematical modeling of micropolar fluid flow through an overlapping arterial stenosis", Int. J. Biomath. 8:4 (2015), 1550056.

[Hogan and Henriksen 1989] H. A. Hogan and M. Henriksen, "An evaluation of a micropolar model for blood flow through an idealized stenosis", J. Biomech. 22:3 (1989), 211-218.

[Ivanova and Vilchevskaya 2016] E. A. Ivanova and E. N. Vilchevskaya, "Micropolar continuum in spatial description”, Contin. Mech. Thermodyn. 28:6 (2016), 1759-1780.

[Kazakia and Ariman 1971] Y. Kazakia and T. Ariman, "Heat-conducting micropolar fluids", Rheol. Acta 10:3 (1971), 319-325.

[Kirwan 1986] A. D. Kirwan, Jr., "Boundary conditions for micropolar fluids", Int. J. Engrg. Sci. 24:7 (1986), 1237-1242.

[Kirwan and Newman 1969a] A. D. Kirwan, Jr. and N. Newman, "Plane flow of a fluid containing rigid structures", Int. J. Engrg. Sci. 7:8 (1969), 883-893.

[Kirwan and Newman 1969b] A. D. Kirwan, Jr. and N. Newman, "Simple flow of a fluid containing deformable structures", Int. J. Engrg. Sci. 7:10 (1969), 1067-1078.

[Kolpashchikov et al. 1983] V. L. Kolpashchikov, N. P. Migun, and P. P. Prokhorenko, "Experimental determination of material micropolar fluid constants", Int. J. Engrg. Sci. 21:4 (1983), 405-411.

[Kucaba-Pietal 2004] A. Kucaba-Pietal, "Microchannels flow modelling with the micropolar fluid theory", B. Pol. Acad. Sci. Tech. 52:3 (2004), 209-214.

[Kucaba-Pietal et al. 2009] A. Kucaba-Pietal, Z. Walenta, and Z. Peradzyński, "Molecular dynamics computer simulation of water flows in nanochannels", B. Pol. Acad. Sci. Tech. 57:1 (2009), 55-61. 
[Lauga et al. 2007] E. Lauga, M. Brenner, and H. Stone, "Microfluidics: the no-slip boundary condition", Chapter 19, pp. 1219-1240 in Springer handbook of experimental fluid mechanics, edited by C. Tropea et al., Springer, 2007.

[Lopez et al. 2016] M. Lopez, J. Chen, and V. A. Palochko, "A multiscale study of the boundary layer development for microfluidic system", Mol. Simulat. 42:16 (2016), 1370-1378.

[Łukaszewicz 1999] G. Łukaszewicz, Micropolar fluids: theory and applications, Birkhäuser, 1999. [Magyari et al. 2010] E. Magyari, I. Pop, and P. P. Valkó, "Stokes' first problem for micropolar fluids”, Fluid Dyn. Res. 42:2 (2010), 025503.

[Mindlin 1965] R. D. Mindlin, "Second gradient of strain and surface-tension in linear elasticity", Int. J. Solids Struct. 1:4 (1965), 417-438.

[Müller 2007] I. Müller, A history of thermodynamics: the doctrine of energy and entropy, Springer, 2007.

[Müller 2014] W. H. Müller, An expedition to continuum theory, Solid Mechanics and its Applications 210, Springer, 2014.

[Pennington and Cowin 2000] C. J. Pennington and S. C. Cowin, "Couette flow of a polar fluid", $T$. Soc. Rheol. 13:3 (2000), 387-403.

[Sheikholeslami et al. 2014] M. Sheikholeslami, M. Hatami, and D. D. Ganji, "Micropolar fluid flow and heat transfer in a permeable channel using analytical method", J. Mol. Liq. 194 (2014), 30-36.

[Silber et al. 2007] G. Silber, U. Janoske, M. Alizadeh, and G. Benderoth, "An application of a gradient theory with dissipative boundary conditions to fully developed turbulent flow", J. Fluids Engrg. 129:5 (2007), 643-651.

[Singh 1982] K. Singh, "Couette flow of microthermopolar fluids between two parallel plates", Acta Mech. 43:1-2 (1982), 1-13.

[Steward 2004] I. W. Steward, The static and dynamic continuum theory of liquid crystals: a mathematical introduction, Taylor \& Francis, 2004.

[Verma and Sehgal 1968] P. D. S. Verma and M. M. Sehgal, "Couette flow of micropolar fluids", Int. J. Engrg. Sci. 6:4 (1968), 233-238.

[Řiha 1975] P. Řiha, "On the theory of heat-conducting micropolar fluids with microtemperatures", Acta Mech. 23:1-2 (1975), 1-8.

[Walter 1998] W. Walter, Ordinary differential equations, Graduate Texts in Math. 182, Springer, 1998.

[Zhilin 2012] P. A. Zhilin, Rational continuum mechanics, Saint Petersburg Polytechnic University, 2012. In Russian.

[Zueger and Lakes 2016] Z. Zueger and R. S. Lakes, "Experimental Cosserat elasticity in open-cell polymer foam”, Philos. Mag. 96:2 (2016), 93-111.

Received 8 Jun 2018. Revised 16 Aug 2018. Accepted 20 Sep 2018.

WILHELM RICKERT: rickert@tu-berlin.de

Institut für Mechanik, Fachgebiet Kontinuumsmechanik und Materialtheorie,

Technische Universität Berlin, Berlin, Germany

ELENA N. VILCHEVSKAYA: vilchevska@gmail.com

Applied Research Laboratory, Institute for Problems in Mechanical Engineering,

Russian Academy of Sciences, Saint Petersburg, Russia

WOLFGANG H. MÜLLER: wolfgang.h.mueller@tu-berlin.de

Institut für Mechanik, Fachgebiet Kontinuumsmechanik und Materialtheorie,

Technische Universität Berlin, Berlin, Germany 


\title{
ANALYTICAL SOLUTIONS FOR THE NATURAL FREQUENCIES OF RECTANGULAR SYMMETRIC ANGLE-PLY LAMINATED PLATES
}

\author{
Florence BROWNING AND HARM ASKES
}

\begin{abstract}
Analytical solutions, based on the Ritz method, are derived for the lowest natural frequency of rectangular symmetric angle-ply laminated plates. Since symmetric angle-ply plates have nonzero cross-elasticity constants, the solutions are approximate. The accuracy of these solutions is tested with a convergence study using the Rayleigh quotient iteration method. With the solutions available in symbolic form, parameter studies are presented that establish the effect of plate aspect ratio and ply orientation angle for a number of stacking geometries. The results are also verified through a comparison with numerical Ritz solutions, showing a maximum error of $5 \%$ in our approximate solution.
\end{abstract}

\section{Introduction}

The focus of this paper is on the natural frequencies of rectangular anisotropic plates. Such plates often consist of layers the principal directions of which are aligned with the edges of the plates, but this restriction is not necessary - indeed, here we will focus on alternative arrangements. The stacking geometry affects the elastic properties of laminated plates, of which the lowest natural frequency is of particular relevance for serviceability criteria [Gsell et al. 2007].

To make qualitative and quantitative predictions of the mechanical behaviour of laminated plates, appropriate plate theory needs to be formulated. Starting from Kirchhoff-Love or Reissner-Mindlin theory, the heterogeneous effects of multiple layers of anisotropic material can be homogenised to arrive at an equivalent anisotropic plate theory; see for instance [Yang et al. 1966; Whitney and Leissa 1969; Leissa and Whitney 1970; Whitney 1972; Pagano 1969; 1970; Kulkarni and Pagano 1972]. These anisotropic plate models were discussed and compared to other modelling strategies for plates (and shells) by Noor et al. [1996]. Further refinements have also been developed; see for instance the 1980s work [Reddy 1984;

\section{Communicated by Francesco dell'Isola.}

PACS2010: 43.40.+s, 46.40.-f.

MSC2010: 74G10, 74H45.

Keywords: laminated plate, natural frequencies, anisotropy, elasticity. 
Murakami 1986; Ren 1986] and the comparative studies [Carrera 2000; Stürzenbecher et al. 2010] of more recent years. The natural frequencies of laminated plates have been studied by Ohta and Ikuno [2002], who restricted their study to so-called cross-laminated plates (whereby the principal directions of each ply are parallel to the plate edges), and by Chaudhuri [2002] and Huang et al. [2006], who developed series-based analytical solutions that can be used for arbitrary layer geometries.

An aspect that has received relatively little attention in the literature (but for exceptions see [Hohe 2013]) is the effect of layer orientation on the natural frequencies of laminated plates, and in that context this paper will focus on so-called angle-ply plates, whereby every layer with a particular fibre orientation has a counterpart layer with the opposite fibre orientation. First, the relevant equations will be summarised briefly. The case of symmetric angle-ply plates is of interest, as the coupling of normal stresses to shear strains and vice versa leads to additional complexity that has, as far as the authors are aware, prohibited the establishment of a closed-form exact solution to date. Additional assumptions are required to formulate a simple approximate analytical solution; the validity of these assumptions is checked in a convergence study carried out in symbolic form with the Rayleigh quotient iteration method, which resulted in a novel set of Padé approximations. The results are also compared to numerical solutions obtained with the Ritz method, and a good agreement between the simple analytical solution and the two series solutions was observed. The usefulness and novelty of these solutions relies on their transparency - whilst the series-based solutions presented in [Chaudhuri 2002; Huang et al. 2006] can be expanded to arbitrary accuracy, they comprise lengthy expressions. For practical considerations and straightforward parameter studies a simpler solution with an upper limit to the error is often preferred.

\section{Anisotropic plate theory}

The anisotropic plate theory used in this paper builds on the seminal works of Whitney, Pagano, and their coworkers from the late 1960s and early 1970s. Membrane action and distributed loads will be ignored. Rectangular plates of length $A$, width $B$, and thickness $H$ will be considered using the global Cartesian coordinate system shown in Figure 1. We will also define the aspect ratio $\alpha$ as $\alpha=A / B$.

For every individual ply, a local 1-2 coordinate system can be introduced whereby the 1 -axis is aligned with the fibres, rotated along an angle $\theta$ from the $x$-axis. The stress-strain relation in local coordinates reads

$$
\left[\begin{array}{c}
\sigma_{1} \\
\sigma_{2} \\
\tau_{12}
\end{array}\right]=\left[\begin{array}{ccc}
Q_{11} & Q_{12} & 0 \\
Q_{12} & Q_{22} & 0 \\
0 & 0 & Q_{66}
\end{array}\right]\left[\begin{array}{c}
\varepsilon_{1} \\
\varepsilon_{2} \\
\gamma_{12}
\end{array}\right] .
$$




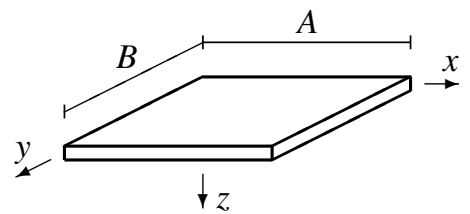

Figure 1. Global coordinate system and plate dimensions.

The constitutive coefficients $Q_{i j}$ are written in terms of elastic constants as

$$
\begin{aligned}
Q_{11} & =\frac{E_{1}}{1-v_{12} v_{21}}, \\
Q_{12} & =\frac{v_{12} E_{2}}{1-v_{12} v_{21}}=\frac{v_{21} E_{1}}{1-v_{12} v_{21}}, \\
Q_{22} & =\frac{E_{2}}{1-v_{12} v_{21}}, \\
Q_{66} & =G_{12},
\end{aligned}
$$

where $E_{1}$ and $E_{2}$ are the Young's moduli parallel and perpendicular to the fibre direction, $G_{12}$ is the shear modulus, and $\nu_{12}$ and $\nu_{21}$ are the Poisson's ratios associated with a stretch in the 1-direction and 2-direction, respectively.

The local stress-strain relations are translated to the global coordinate system, leading to

$$
\left[\begin{array}{l}
\sigma_{x x} \\
\sigma_{y y} \\
\tau_{x y}
\end{array}\right]=\left[\begin{array}{lll}
\bar{Q}_{11} & \bar{Q}_{12} & \bar{Q}_{16} \\
\bar{Q}_{12} & \bar{Q}_{22} & \bar{Q}_{26} \\
\bar{Q}_{16} & \bar{Q}_{26} & \bar{Q}_{66}
\end{array}\right]\left[\begin{array}{l}
\varepsilon_{x x} \\
\varepsilon_{y y} \\
\gamma_{x y}
\end{array}\right],
$$

where

$$
\begin{aligned}
& \bar{Q}_{11}=Q_{11} \cos ^{4} \theta+\left(2 Q_{12}+4 Q_{66}\right) \cos ^{2} \theta \sin ^{2} \theta+Q_{22} \sin ^{4} \theta, \\
& \bar{Q}_{22}=Q_{11} \sin ^{4} \theta+\left(2 Q_{12}+4 Q_{66}\right) \cos ^{2} \theta \sin ^{2} \theta+Q_{22} \cos ^{4} \theta, \\
& \bar{Q}_{12}=\left(Q_{11}+Q_{22}-2 Q_{12}-4 Q_{66}\right) \cos ^{2} \theta \sin ^{2} \theta+Q_{12}, \\
& \bar{Q}_{66}=\left(Q_{11}+Q_{22}-2 Q_{12}-4 Q_{66}\right) \cos ^{2} \theta \sin ^{2} \theta+Q_{66}, \\
& \bar{Q}_{16}=\left(Q_{11}-Q_{12}-2 Q_{66}\right) \cos ^{3} \theta \sin \theta+\left(Q_{12}-Q_{22}+2 Q_{66}\right) \cos \theta \sin ^{3} \theta, \\
& \bar{Q}_{26}=\left(Q_{11}-Q_{12}-2 Q_{66}\right) \cos \theta \sin ^{3} \theta+\left(Q_{12}-Q_{22}+2 Q_{66}\right) \cos ^{3} \theta \sin \theta .
\end{aligned}
$$

Note that the above transformation is expressed in terms of the engineering shear strain, not the tensorial shear strain, meaning that additional factors 2 and $\frac{1}{2}$ have been used compared to the usual transformation rules of second-order tensors.

The constitutive coefficients (4) are assumed to be piecewise constant for each ply. The heterogeneous response in the $z$-direction is homogenised by integrating 
the stresses over the thickness of the plate, leading to the equation of transverse motion

$$
\begin{aligned}
D_{11} \frac{\partial^{4} w}{\partial x^{4}}+\left(2 D_{12}+4 D_{66}\right) \frac{\partial^{4} w}{\partial x^{2} \partial y^{2}} & +D_{22} \frac{\partial^{4} w}{\partial y^{4}} \\
& +4 D_{16} \frac{\partial^{4} w}{\partial x^{3} \partial y}+4 D_{26} \frac{\partial^{4} w}{\partial x \partial y^{3}}+\rho H \frac{\partial^{2} w}{\partial t^{2}}=0,
\end{aligned}
$$

where $w$ is the transverse displacement, $\rho$ is the mass density, and $H$ is the total thickness of the plate. Furthermore, the various plate bending coefficients $D_{i j}$ are defined by

$$
D_{i j}=\int_{-H / 2}^{H / 2} \bar{Q}_{i j} z^{2} d z
$$

In case $D_{16} \neq 0$ and $D_{26} \neq 0$, an additional level of anisotropy is obtained in going from (1) to (3). These two coefficients are called the "cross-elasticity bending stiffness terms" [Whitney 1972]. In (5) this manifests itself in odd-order derivatives in terms of $x$ and $y$, which has some implications for subsequent derivations, as will be explained below.

The boundary conditions for simply supported rectangular plates read

$$
\begin{aligned}
& w=0, \quad M_{y}=-D_{11} \frac{\partial^{2} w}{\partial x^{2}}-2 D_{16} \frac{\partial^{2} w}{\partial x \partial y}-D_{12} \frac{\partial^{2} w}{\partial y^{2}}=0 \quad \text { at } x=0, A, \\
& w=0, \quad M_{x}=-D_{12} \frac{\partial^{2} w}{\partial x^{2}}-2 D_{26} \frac{\partial^{2} w}{\partial x \partial y}-D_{22} \frac{\partial^{2} w}{\partial y^{2}}=0 \quad \text { at } y=0, B,
\end{aligned}
$$

where $M_{x}$ and $M_{y}$ are the distributed moments (per unit of length) about the $x$-axis and $y$-axis, respectively.

\section{Plate bending coefficients of symmetric angle-ply plates}

In so-called angle-ply plates, each layer with orientation angle $\theta$ has a counterpart layer with orientation angle $-\theta$. Typical stacking sequences are symmetric or antisymmetric around the midplane $z=0$. We will assume symmetric angle-ply plates with multiples of four layers in total, the same thickness for each layer, and alternating orientations of $+\theta$ and $-\theta$ for consecutive layers in each of the top half and bottom half of the plate. The standard plate bending coefficients can then be written as

$$
\begin{aligned}
& D_{11}=\frac{H^{3}}{12}\left(Q_{11} \cos ^{4} \theta+\left(2 Q_{12}+4 Q_{66}\right) \cos ^{2} \theta \sin ^{2} \theta+Q_{22} \sin ^{4} \theta\right), \\
& D_{22}=\frac{H^{3}}{12}\left(Q_{11} \sin ^{4} \theta+\left(2 Q_{12}+4 Q_{66}\right) \cos ^{2} \theta \sin ^{2} \theta+Q_{22} \cos ^{4} \theta\right),
\end{aligned}
$$




$$
\begin{aligned}
& D_{12}=\frac{H^{3}}{12}\left(\left(Q_{11}+Q_{22}-2 Q_{12}-4 Q_{66}\right) \cos ^{2} \theta \sin ^{2} \theta+Q_{12}\right), \\
& D_{66}=\frac{H^{3}}{12}\left(\left(Q_{11}+Q_{22}-2 Q_{12}-4 Q_{66}\right) \cos ^{2} \theta \sin ^{2} \theta+Q_{66}\right)
\end{aligned}
$$

irrespective of the actual number of layers, while the cross-elasticity plate bending coefficients read

$$
\begin{aligned}
& D_{16}=\frac{H^{3} \sin 2 \theta}{8 \cdot n_{\text {layer }}}\left(\left(Q_{11}-2 Q_{12}+Q_{22}-4 Q_{66}\right) \cos ^{2} \theta-Q_{22}+Q_{12}+2 Q_{66}\right), \\
& D_{26}=-\frac{H^{3} \sin 2 \theta}{8 \cdot n_{\text {layer }}}\left(\left(Q_{11}-2 Q_{12}+Q_{22}-4 Q_{66}\right) \cos ^{2} \theta-Q_{11}+Q_{12}+2 Q_{66}\right)
\end{aligned}
$$

where $n_{\text {layer }}$ is the total number of layers. It can be seen that the cross-elasticity coefficients vanish when the fibre orientations coincide with the Cartesian axes. Furthermore, the magnitude of the cross-elasticity coefficients reduces with an increased number of layers. Thus, the maximum effect of cross-elasticity is obtained for four layers, which will be assumed in the remainder of the paper.

\section{Symmetric square angle-ply plate - a convergence study}

In the presence of cross-elasticity, i.e., $D_{16} \neq 0$ and $D_{26} \neq 0$, it has been mentioned that an exact solution is not available [Jones 1999, p. 318]. Analytical solutions have been developed in [Chaudhuri 2002] and [Huang et al. 2006], but they are expressed in an extended series format, and a symbolic representation does not seem to be sufficiently transparent to be practicable. Instead, we have opted to use the Ritz method in symbolic form to generate an approximate solution to the cross-elasticity case.

The general solution for the Ritz method in the case of simply supported plates reads

$$
w(x, y, t)=\sin (\omega t) \sum_{m=1}^{M} \sum_{n=1}^{N} C_{m, n} \sin \frac{m \pi x}{A} \sin \frac{n \pi y}{B} .
$$

Adopting a square geometry, i.e., $A=B$, and using the same number of terms in both the $x$ and $y$ direction, i.e., $M=N$, the exact solutions of the Ritz problem for the lowest two values of $M$ and $N$ are denoted by $\omega_{(M, N)}^{2}$ and read

$$
\begin{aligned}
& \omega_{(1,1)}^{2}=\frac{\pi^{4} H^{2} Q_{a a}}{12 \rho B^{4}}, \\
& \omega_{(2,2)}^{2}=\frac{\pi^{4} H^{2} Q_{a a}}{12 \rho B^{4}} \cdot\left(\frac{17}{2}-\frac{15}{2} \sqrt{1+\frac{1024}{81} \frac{Q_{b b}^{2}}{Q_{a a}^{2}}}\right)
\end{aligned}
$$


where

$$
\begin{aligned}
Q_{a a} & =Q_{11}+2 Q_{12}+Q_{22}+4 Q_{66}+\left(Q_{11}-2 Q_{12}+Q_{22}-4 Q_{66}\right) \sin ^{2} 2 \theta, \\
Q_{b b} & =\left(Q_{11}-Q_{22}\right) \frac{\sin 2 \theta}{\pi^{2}}
\end{aligned}
$$

have been defined for a more compact notation.

For larger values of $M$ and $N$, the size of the stiffness matrix becomes prohibitive to finding an exact solution in transparent symbolic form; thus, we have made two further approximations. Firstly, we have discarded any terms whereby $m \neq n-$ this can be justified by inspecting the eigenvector associated with (10b). Secondly, we have applied two iterations of the Rayleigh quotient iteration method to arrive at a relatively simple closed-form approximation of the lowest natural frequency.

(1) With trial eigenvector $\underline{v}_{1}=[1,0,0, \ldots]^{T}$ we have computed the Rayleigh quotient RQ according to

$$
\mathrm{RQ}=\frac{\underline{v}^{T} \boldsymbol{K} \underline{v}}{\underline{v}^{T} \boldsymbol{M} \underline{v}}
$$

where $\boldsymbol{K}$ and $\boldsymbol{M}$ are the stiffness matrix and mass matrix, respectively. Not surprisingly, the value of the Rayleigh quotient in this first iteration equals the expression for $\omega^{2}$ given in (10a).

(2) These values for $\underline{v}$ and RQ are used to compute an update on $\underline{v}$ according to

$$
\underline{v}_{2}=[\boldsymbol{K}-\mathrm{RQ} \cdot \boldsymbol{M}]^{-1} \underline{v}_{1}
$$

after which this new estimate of the eigenvector is used to recompute the Rayleigh quotient, and the resulting value of RQ is taken as an approximation for $\omega^{2}$. Carrying out further iterations in symbolic form is possible in principle, but leads to expressions that are too unwieldy to be of practical use.

The above procedure has been executed for $M=N=2$ up to $M=N=5$, which gives the results in Table 1 on the next page. The first of these expressions, (15a), has been included to provide a comparison with the Ritz solution of (10b); the former is also a [1,1]-Padé approximation of the latter, following $\sqrt{1+x} \approx(4+3 x) /(4+x)$.

To estimate the differences between the various solutions, it is first established that the ratio $Q_{b b} / Q_{a a}$ adopts its maximum value for $\theta=\frac{1}{4} \pi$; in particular

$$
\frac{Q_{b b}}{Q_{a a}}=\frac{1}{2 \pi^{2}} \cdot \frac{E_{1}-E_{2}}{E_{1}+E_{2}} \quad \text { at } \theta=\frac{1}{4} \pi,
$$

which in turn adopts its maximum value for vanishing $E_{2}$. With the estimate $Q_{b b} / Q_{a a}<1 / 2 \pi^{2}$, the relative difference between (15a) and (10b) is less than 


$$
\begin{aligned}
\omega_{(2,2)}^{2} \approx & \frac{\pi^{4} H^{2} Q_{a a}}{12 \rho B^{4}} \cdot \frac{81-3584 Q_{b b}^{2} / Q_{a a}^{2}}{81+256 Q_{b b}^{2} / Q_{a a}^{2}}, \\
\omega_{(3,3)}^{2} \approx & \frac{\pi^{4} H^{2} Q_{a a}}{12 \rho B^{4}} \cdot \frac{1-128 Q_{b b}^{2} / Q_{a a}^{2}+3787 Q_{b b}^{4} / Q_{a a}^{4}}{1-81 Q_{b b}^{2} / Q_{a a}^{2}+1793 Q_{b b}^{4} / Q_{a a}^{4}}, \\
\omega_{(4,4)}^{2} \approx & \frac{\pi^{4} H^{2} Q_{a a}}{12 \rho B^{4}} \cdot \frac{1-205 Q_{b b}^{2} / Q_{a a}^{2}+11457 Q_{b b}^{4} / Q_{a a}^{4}-103802 Q_{b b}^{6} / Q_{a a}^{6}}{1-157 Q_{b b}^{2} / Q_{a a}^{2}+6251 Q_{b b}^{4} / Q_{a a}^{4}+3625 Q_{b b}^{6} / Q_{a a}^{6}}, \\
\omega_{(5,5)}^{2} \approx & \frac{\pi^{4} H^{2} Q_{a a}}{12 \rho B^{4}} . \\
& \frac{1-281 Q_{b b}^{2} / Q_{a a}^{2}+24601 Q_{b b}^{4} / Q_{a a}^{4}-681845 Q_{b b}^{6} / Q_{a a}^{6}+5045965 Q_{b b}^{8} / Q_{a a}^{8}}{1-233 Q_{b b}^{2} / Q_{a a}^{2}+15876 Q_{b b}^{4} / Q_{a a}^{4}-266394 Q_{b b}^{6} / Q_{a a}^{6}+1438407 Q_{b b}^{8} / Q_{a a}^{8}} .
\end{aligned}
$$

Table 1. Approximate natural frequencies for the Ritz problem in the cross-elasticity case: symmetric square, modes with $M=N$ ranging from 2 through 5 .

$10^{-5}$, which indicates that the Rayleigh quotient iteration method provides an excellent approximation of the Ritz solution. Comparing (15b), (15c), and (15d) to (10b) leads to maximum relative errors of $1.5 \%, 2.4 \%$, and $2.7 \%$, respectively.

These results are obviously encouraging, but they must be interpreted with some caution. Whilst it is well-known that the Rayleigh quotient iteration method converges very rapidly (also confirmed by the comparison above for $M=N=2$ ), it must be kept in mind that these results are obtained using two iterations only. Furthermore, the Ritz method itself is known to converge much slower than other methods such as direct Fourier series [Whitney 1972], although convergence is better for plates that are simply supported on all sides. Applying the Rayleigh quotient iteration method in symbolic form could be used to increase the accuracy of the analytical solution, but instead of seeking a solution in series format we will next use the Ritz method with $M=N=2$ below to extend the analysis to rectangular plates.

\section{Rectangular angle-ply plates}

Using (9) with $M=N=2$ leads to a stiffness matrix $\boldsymbol{K}$ and mass matrix $\boldsymbol{M}$ as

$$
\boldsymbol{K}=\left[\begin{array}{cccc}
K_{11} & 0 & 0 & K_{14} \\
0 & K_{22} & K_{23} & 0 \\
0 & K_{23} & K_{33} & 0 \\
K_{14} & 0 & 0 & K_{44}
\end{array}\right] \text { and } \boldsymbol{M}=\frac{\rho H B^{2} \alpha}{4} \boldsymbol{I}
$$

where $I$ is the identity matrix. Noting that $K_{44}=16 K_{11}$, the lowest natural 


\begin{tabular}{|c|cccccc|}
\hline & $E_{1}[\mathrm{GPa}]$ & $E_{2}[\mathrm{GPa}]$ & $v_{12}$ & $v_{21}$ & $G_{12}[\mathrm{GPa}]$ & $\rho\left[\mathrm{kg} / \mathrm{m}^{3}\right]$ \\
\hline GFR composite & 46.2 & 16.6 & 0.26 & 0.093 & 6.9 & 2027 \\
spruce & 11.0 & 0.37 & 0.44 & 0.015 & 0.69 & 500 \\
\hline
\end{tabular}

Table 2. Material properties for a glass-fibre reinforced composite and spruce.

frequency is found from

$$
\omega=\sqrt{\frac{34 K_{11}-2 \sqrt{225 K_{11}^{2}+4 K_{14}^{2}}}{\rho H B^{2} \alpha}}
$$

where the relevant stiffness matrix components are given in terms of the plate bending coefficients as

$$
\begin{aligned}
& K_{11}=\frac{\pi^{4}}{4 B^{2} \alpha^{3}}\left(D_{11}+\left(2 D_{12}+4 D_{66}\right) \alpha^{2}+D_{22} \alpha^{4}\right), \\
& K_{14}=-\frac{160 \pi^{2}}{9 B^{2} \alpha^{2}}\left(D_{16}+D_{26} \alpha^{2}\right) .
\end{aligned}
$$

For quantitative comparisons, we will use the two sets of material parameters listed in Table 2, associated respectively with a glass-fibre reinforced (GFR) composite [Fällström et al. 1996] and spruce [Stürzenbecher et al. 2010].

The natural frequencies according to (17) have been plotted in Figure 2 for a
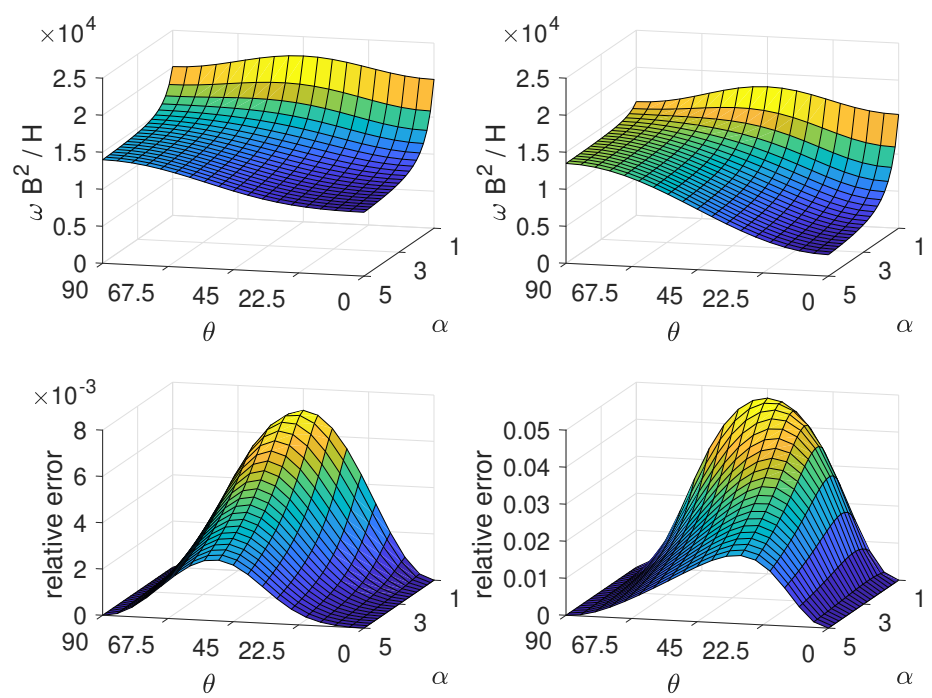

Figure 2. Normalised natural frequency $\omega$ (top) and relative error of the analytical solution (bottom) against plate aspect ratio $\alpha$ and fibre orientation angle $\theta$. Left: GFR composite; right: spruce. 
range of plate aspect ratios and fibre orientation angles, and for the two sets of material parameters given in Table 2 - note that the fibre orientation angle is plotted in degrees, not radians. The approximate solutions of (17) have also been compared to solutions obtained numerically with the Ritz method using $M=N=50$.

The natural frequencies for the two sets of material data are qualitatively very similar. The maximum frequency is found for $\theta=\frac{1}{4} \pi$ in case of aspect ratios close to unity, and for $\theta=\frac{1}{2} \pi$ in case of larger aspect ratios. The dependence on the aspect ratio is strongest for $\theta=0$, i.e., when the fibres are spanning the larger dimension - particularly for spruce, which has a larger $E_{1} / E_{2}$ ratio. The quantitative differences between the GFR composite and spruce are due to the much stronger degree of anisotropy encountered in spruce. This also impacts the accuracy of the approximate solution given in (17). The relative error of the analytical solution with respect to the benchmark numerical solution is seen to be less than $1 \%$ for the GFR composite, but more than five times as high for spruce. Nevertheless, a $5 \%$ error only occurs for aspect ratio $\alpha=1$ and fibre orientations $\theta=\frac{1}{4} \pi$ (which is also the geometry for which maximum errors were studied in Section 4), whereas other combinations of $\alpha$ and $\theta$ lead to (much) lower errors. This level of accuracy is deemed to be proportionate and acceptable, given the simplicity and transparency of (17) and (18).

\section{Conclusions}

Simple and transparent expressions for the lowest natural frequency have been derived for rectangular anisotropic plates. Since the plate behaviour includes a coupling between normal stresses and shear strains (and vice versa), a phenomenon known as "cross-elasticity", the solutions are approximate. A rudimentary convergence study in symbolic form based on the Rayleigh quotient iteration method has confirmed that a relatively low order of the Ritz method can be used for cases that are simply supported on all sides. This has been employed for more general plate configurations with cross-elasticity; because the solutions are obtained in symbolic form, parameter studies are straightforward. The accuracy of the method has been further confirmed by comparison with numerically obtained Ritz solutions.

Due to the chosen approach of seeking closed-form solutions, certain simplifications and assumptions had to be made. Thus, we have studied only one set of boundary conditions, namely simply supported on all sides, we have ignored membrane action, and cross-sectional warping has not been included. These effects, and others, can be studied using a numerical solution approach in combination with a more sophisticated plate theory, whereby the analytical solutions provided in this paper may serve as reference solutions. 


\section{Acknowledgements}

We are indebted to Doctor Karin de Borst (Shell, The Netherlands) and Professor Ilanko (University of Waikato, New Zealand) for kindly providing insightful comments on an earlier draft.

\section{References}

[Carrera 2000] E. Carrera, "An assessment of mixed and classical theories on global and local response of multilayered orthotropic plates", Compos. Struct. 50:2 (2000), 183-198.

[Chaudhuri 2002] R. A. Chaudhuri, "On the roles of complementary and admissible boundary constraints in Fourier solutions to the boundary value problems of completely coupled $r$ th order PDEs", J. Sound Vib. 251:2 (2002), 261-313.

[Fällström et al. 1996] K.-E. Fällström, K. Olofsson, H. O. Saldner, and S. Schedin, "Dynamic material parameters in an anisotropic plate estimated by phase-stepped holographic interferometry", Opt. Laser. Eng. 24:5-6 (1996), 429-454.

[Gsell et al. 2007] D. Gsell, G. Feltrin, S. Schubert, R. Steiger, and M. Motavalli, "Cross-laminated timber plates: evaluation and verification of homogenized elastic properties", J. Struct. Eng. 133:1 (2007), 132-138.

[Hohe 2013] J. Hohe, "Effect of core and face sheet anisotropy on the natural frequencies of sandwich shells with composite faces", Int. J. Compos. Mater. 3:6B (2013), 40-52.

[Huang et al. 2006] Y. Huang, Y. J. Lei, and H. J. Shen, "Free vibration of anisotropic rectangular plates by general analytical method", Appl. Math. Mech. 27:4 (2006), 461-467.

[Jones 1999] R. M. Jones, Mechanics of composite materials, 2nd ed., Taylor \& Francis, New York, 1999.

[Kulkarni and Pagano 1972] S. V. Kulkarni and N. J. Pagano, "Dynamic characteristics of composite laminates", J. Sound. Vib. 23:1 (1972), 127-143.

[Leissa and Whitney 1970] A. W. Leissa and J. M. Whitney, "Analysis of a simply supported laminated anisotropic rectangular plate", AIAA J. 8:1 (1970), 28-33.

[Murakami 1986] H. Murakami, "Laminated composite plate theory with improved in-plane responses", J. Appl. Mech. 53:3 (1986), 661-666.

[Noor et al. 1996] A. K. Noor, W. S. Burton, and C. W. Bert, "Computational models for sandwich panels and shells", Appl. Mech. Rev. 49:3 (1996), 155-199.

[Ohta and Ikuno 2002] Y. Ohta and T. Ikuno, "The study of analytical models for vibration of crossply laminated thick plates", JSME Int. J. C 45:1 (2002), 107-112.

[Pagano 1969] N. J. Pagano, "Exact solutions for composite laminates in cylindrical bending", $J$. Compos. Mater. 3:3 (1969), 398-411.

[Pagano 1970] N. J. Pagano, "Exact solutions for rectangular bidirectional composites and sandwich plates”, J. Compos. Mater. 4:1 (1970), 20-34.

[Reddy 1984] J. N. Reddy, "A simple higher-order theory for laminated composite plates", J. Appl. Mech. 51:4 (1984), 745-752.

[Ren 1986] J. G. Ren, "A new theory of laminated plate”, Compos. Sci. Tech. 26:3 (1986), 225-239.

[Stürzenbecher et al. 2010] R. Stürzenbecher, K. Hofstetter, and J. Eberhardsteiner, "Structural design of Cross Laminated Timber (CLT) by advanced plate theories", Compos. Sci. Tech. 70:9 (2010), $1368-1379$. 
[Whitney 1972] J. M. Whitney, "On the analysis of anisotropic rectangular plates", technical report AD-776017, Air Force Materials Laboratory, 1972, Available at http://www.dtic.mil/dtic/tr/fulltext/ u2/776017.pdf.

[Whitney and Leissa 1969] J. M. Whitney and A. W. Leissa, "Analysis of heterogeneous anisotropic plates", J. Appl. Mech. 36:2 (1969), 261-266.

[Yang et al. 1966] P. C. Yang, C. H. Norris, and Y. Stavsky, "Elastic wave propagation in heterogeneous plates", Int. J. Solid. Struct. 2:4 (1966), 665-684.

Received 17 Jul 2018. Revised 29 Aug 2018. Accepted 24 Oct 2018.

FLORENCE BROWNING: florence.browning22@gmail.com

Interrobang Architecture and Engineering, Webb Yates Engineers Ltd, London, United Kingdom

HARM ASKES: h.askes@sheffield.ac.uk

Department of Civil and Structural Engineering, University of Sheffield, Sheffield, United Kingdom

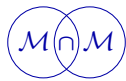





\title{
ON THE BLOCKING LIMIT OF STEADY-STATE FLOW OF HERSCHEL-BULKLEY FLUID
}

\author{
FARID MESSELMI
}

\begin{abstract}
This paper is devoted to the study of the blocking limit of Herschel-Bulkley fluid in the case of steady-state flow. To this aim, we consider a mathematical model which describes the steady-state flow of a Herschel-Bulkley fluid in a bounded domain. We give the mathematical formulation of the blockage phenomenon, and we establish the existence of the blocking limit. We also focus on behaviour of the flow with respect to the blocking limit.
\end{abstract}

\section{Introduction}

The rigid viscoplastic and incompressible fluid of Herschel and Bulkley has been investigated by mathematicians, physicists, and engineers as intensively as the Navier-Stokes equations though this model adequately describes a large class of flows. It has been used to model the flow of metals, plastic solids, and a variety of polymers. Physical experiments and numerical studies of the flow of HerschelBulkley fluids prove that when the yield stress increases, the rigid zones become larger and may completely block the flow. This property is called the blocking phenomenon. Due to existence of the yield limit, the model can capture phenomena connected with the development of discontinuous stresses. The literature concerning this topic is extensive; see, e.g., [Málek 2008; Málek et al. 2006; 2005; Messelmi 2017; Messelmi and Merouani 2013; 2010; Messelmi et al. 2010].

Our paper deals with the steady-state flow of Herschel and Bulkley. The main objective is the study of the behaviour of the flow. We provide a generalisation of a result obtained by Hild et al. [2002] for Bingham fluid to the steady-state flow of the Herschel-Bulkley model, ensuring the existence of the blocking limit. Moreover, we establish a result concerning the behaviour of the flow when the yield limit is near a minimal blocking limit.

The paper is organised as follows. In Section 2 we present the mechanical problem of the steady-state flow of Herschel-Bulkley fluid in a bounded domain $\Omega \subset \mathbb{R}^{n}$. We introduce some notation and preliminaries. In addition, we derive

\section{Communicated by Carlo Marchioro.}

MSC2010: 35J85, 76A05, 76E30.

Keywords: blocking limit, Herschel-Bulkley fluid, variational inequality. 
the variational formulation of the problem. In Section 3, we show the mathematical formulation of the blockage phenomenon and we prove the existence of the blocking limit. Section 4 is devoted to the study of the behaviour of the flow with respect to the blocking limit.

\section{Problem statement}

We consider a mathematical problem modelling the steady-state flow of the rigid viscoplastic and incompressible Herschel-Bulkley fluid in a bounded domain $\Omega \subset$ $\mathbb{R}^{n}(n=2,3)$, with the boundary $\Gamma$ of class $C^{1}$. The fluid is acted upon by given volume forces of density $f$. On $\Gamma$ we suppose that the velocity is equal to zero.

We denote by $S_{n}$ the space of symmetric tensors on $\mathbb{R}^{n}$. We define the inner product and the Euclidean norm on $\mathbb{R}^{n}$ and $S_{n}$, respectively, by

$$
\begin{aligned}
& \boldsymbol{u} \cdot \boldsymbol{v}=u_{i} v_{i} \quad \text { for all } \boldsymbol{u}, \boldsymbol{v} \in \mathbb{R}^{n}, \quad \boldsymbol{\sigma} \cdot \boldsymbol{\tau}=\sigma_{i j} \tau_{i j} \quad \text { for all } \boldsymbol{\sigma}, \boldsymbol{\tau} \in S_{n}, \\
& |\boldsymbol{u}|=(\boldsymbol{u} \cdot \boldsymbol{u})^{1 / 2} \quad \text { for all } \boldsymbol{u} \in \mathbb{R}^{n}, \quad|\boldsymbol{\sigma}|=(\boldsymbol{\sigma} \cdot \boldsymbol{\sigma})^{1 / 2} \quad \text { for all } \boldsymbol{\sigma} \in \mathbb{S}_{n} .
\end{aligned}
$$

Here and below, the indices $i$ and $j$ run from 1 to $n$ and the summation convention over repeated indices is used. We denote by $\sigma^{D}$ the deviator of $\sigma=\left(\sigma_{i j}\right)$ given by

$$
\boldsymbol{\sigma}^{D}=\left(\sigma_{i j}^{D}\right), \quad \sigma_{i j}^{D}=\sigma_{i j}-\frac{\sigma_{k k}}{n} \delta_{i j},
$$

where $\boldsymbol{\delta}=\left(\delta_{i j}\right)$ denotes the identity tensor.

Let $1<p \leq 2$. We consider the rate-of-deformation operator defined for every $\boldsymbol{u} \in W^{1, p}(\Omega)^{n}$ by

$$
D(\boldsymbol{u})=\left(D_{i j}(\boldsymbol{u})\right), \quad D_{i j}(\boldsymbol{u})=\frac{1}{2}\left(u_{i, j}+u_{j, i}\right) .
$$

The steady-state flow of Herschel-Bulkley fluid can be described by the following mechanical problem.

Problem $\mathbf{P}_{1}$. Find the velocity field $\boldsymbol{u}=\left(u_{i}\right): \Omega \rightarrow \mathbb{R}^{n}$ and the stress field $\boldsymbol{\sigma}=$ $\left(\sigma_{i j}\right): \Omega \rightarrow S_{n}$ such that

$$
\left.\begin{array}{rlrl}
\boldsymbol{u} \cdot \nabla \boldsymbol{u} & =\operatorname{div} \boldsymbol{\sigma}+\boldsymbol{f} & & \\
\boldsymbol{\sigma}^{D} & =\mu|D(\boldsymbol{u})|^{p-2} D(\boldsymbol{u})+g(D(\boldsymbol{u}) /|D(\boldsymbol{u})|) & & \text { if }|D(\boldsymbol{u})| \neq 0, \\
\left|\boldsymbol{\sigma}^{D}\right| & \leq g & & \text { if }|D(\boldsymbol{u})|=0
\end{array}\right\}
$$

Here $\operatorname{div} \boldsymbol{\sigma}=\left(\sigma_{i j, j}\right)$ and $\operatorname{div} \boldsymbol{u}=u_{i, i}$. The flow is given by (2-1) where the density is assumed equal to one. Equation (2-2) represents the constitutive law of HerschelBulkley fluid where $\mu>0$ and $g \geq 0$ represent the consistency and yield limit of the 
fluid, respectively, and $1<p \leq 2$ is the power law index. Equation (2-3) represents the incompressibility condition. Equation (2-4) gives the adherence condition on the boundary $\Gamma$.

Existence of weak solutions for this problem was proved in 1969 for $p \geq$ $3 n /(n+2)$, for which the energy equality holds and higher differentiability techniques can be applied, in 1997 for $p \geq 2 n /(n+1)$, and recently for $p>2 n /(n+2)$ using the Lipschitz truncation method. Moreover, in 2010 some existence results regarding the thermal flow were established for the case $p \geq 3 n /(n+2)$ [Frehse et al. 2003; Lions 1969; Málek 2008; Málek et al. 2006; Messelmi et al. 2010]. Up to now, there are only a few results concerning the regularity of weak solutions, especially in three-dimensional domains. Further, the asymptotic behaviour of the unsteady flow was the subject of [Messelmi 2017].

Remark. (1) The Bingham fluid represents a particular case of Herschel-Bulkley fluid corresponding to $p=2$.

(2) In the constitutive law of Herschel-Bulkley fluid (2-2), the viscosity and hydrostatic pressure are given, respectively, by

$$
\eta=\mu|D(\boldsymbol{u})|^{p-2}, \quad \pi=-\frac{1}{n} \sigma_{k k} .
$$

Let us introduce the function spaces

$$
\begin{aligned}
W_{p, \operatorname{div}} & =\left\{\boldsymbol{v} \in W_{0}^{1, p}(\Omega)^{n}: \operatorname{div}(\boldsymbol{v})=0 \text { in } \Omega\right\}, \\
L D(\Omega) & =\left\{\boldsymbol{v} \in L^{1}(\Omega)^{n}: D(\boldsymbol{v}) \in L^{1}(\Omega)^{n \times n}\right\}, \\
V D(\Omega) & =\{\boldsymbol{v} \in L D(\Omega): v=0 \text { on } \Gamma\}, \\
W & =\{\boldsymbol{v} \in V D(\Omega): \operatorname{div} \boldsymbol{v}=0 \text { in } \Omega\} .
\end{aligned}
$$

$W_{p, \text { div }}$ is a Banach space equipped with the norm

$$
\|\boldsymbol{v}\|_{W_{p, \text { div }}}=\|\boldsymbol{v}\|_{W^{1, p}(\Omega)^{n}} .
$$

Moreover, Korn's inequality holds in the space $W_{p \text {,div }}$ [Messelmi et al. 2010], which means that there exists a positive constant $C_{0}$ depending only on $\Omega$ and $\Gamma$ such that

$$
C_{0}\|D(\boldsymbol{v})\|_{L^{p}(\Omega)^{n \times n}} \geq\|\boldsymbol{v}\|_{W_{p, \text { div }}} \quad \text { for all } \boldsymbol{v} \in W_{p, \operatorname{div}} .
$$

The space $L D(\Omega)$ was introduced by Temam [1985]. It is a Banach space equipped with the norm

$$
\|\boldsymbol{v}\|_{L D(\Omega)}=\|\boldsymbol{v}\|_{L^{1}(\Omega)^{n}}+\|D(\boldsymbol{v})\|_{L^{1}(\Omega)^{n \times n}},
$$

which is not reflexive, and $W^{1,1}(\Omega)^{n} \subset L D(\Omega)$. Since Korn's inequality does not hold on $L D(\Omega)$ (see the remarks in [Temam 1985]), the space $W^{1,1}(\Omega)^{n}$ is a proper 
subspace of $L D(\Omega) . V D(\Omega)$ is a closed subspace of $L D(\Omega)$. W is also a Banach space equipped with the norm given by (2-12). Furthermore, Korn's inequality holds in $W$ [Temam 1985], and thus, there exists a positive constant $C_{W}$ depending only on $\Omega$ and $\Gamma$ such that

$$
C_{W}\|D(\boldsymbol{v})\|_{L^{1}(\Omega)^{n \times n}} \geq\|\boldsymbol{v}\|_{L D(\Omega)} \quad \text { for all } \boldsymbol{v} \in W .
$$

Denoting by $p^{\prime}$ the conjugate of $p$, we introduce the convective operator

$$
B: W_{p, \operatorname{div}} \times W_{p, \operatorname{div}} \times W_{p, \operatorname{div}} \rightarrow \mathbb{R}, \quad B(\boldsymbol{u}, \boldsymbol{v}, \boldsymbol{w})=\int_{\Omega} \boldsymbol{u} \cdot \nabla \boldsymbol{v} \cdot \boldsymbol{w} d x
$$

We begin by recalling the following lemma [Messelmi et al. 2010], which gives some properties of the convective operator $B$.

Lemma. Suppose that

$$
\frac{3 n}{n+2} \leq p \leq 2
$$

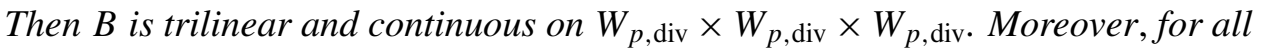
$(\boldsymbol{u}, \boldsymbol{v}, \boldsymbol{w}) \in W_{p, \operatorname{div}} \times W_{p, \operatorname{div}} \times W_{p \text {, div }}$ we have $B(\boldsymbol{u}, \boldsymbol{v}, \boldsymbol{w})=-B(\boldsymbol{u}, \boldsymbol{w}, \boldsymbol{v})$.

For the rest of this paper, we choose $3 n /(n+2) \leq p \leq 2$. The use of Green's formula permits us to derive the following variational formulation of the mechanical problem $\mathrm{P}_{1}$ [Messelmi et al. 2010].

Problem $\mathbf{P}_{g}$. For prescribed data $f \in W_{p \text {, div }}^{\prime}$, find $\boldsymbol{u} \in W_{p \text {,div }}$ satisfying the variational inequality

$$
\begin{aligned}
& B(\boldsymbol{u}, \boldsymbol{u}, \boldsymbol{v}-\boldsymbol{u})+\mu \int_{\Omega}|D(\boldsymbol{u})|^{p-2} D(\boldsymbol{u}) \cdot D(\boldsymbol{v}-\boldsymbol{u}) d x \\
& +g \int_{\Omega}|D(\boldsymbol{v})| d x-g \int_{\Omega}|D(\boldsymbol{u})| d x \geq \int_{\Omega} \boldsymbol{f} \cdot(\boldsymbol{v}-\boldsymbol{u}) d x \quad \text { for all } \boldsymbol{v} \in W_{p, \text { div }}
\end{aligned}
$$

By taking $\boldsymbol{v}=0$ and $\boldsymbol{v}=2 \boldsymbol{u}$ in (2-16), respectively,

$$
\mu \int_{\Omega}|D(\boldsymbol{u})|^{p} d x+g \int_{\Omega}|D(\boldsymbol{u})| d x=\int_{\Omega} \boldsymbol{f} \cdot \boldsymbol{u} d x .
$$

This implies using again (2-16)

$$
\begin{aligned}
B(\boldsymbol{u}, \boldsymbol{u}, \boldsymbol{v})+\mu \int_{\Omega}|D(\boldsymbol{u})|^{p-2} D(\boldsymbol{u}) \cdot D(\boldsymbol{v}) d x+g \int_{\Omega}|D(\boldsymbol{v})| d x & \\
& \geq \int_{\Omega} \boldsymbol{f} \cdot \boldsymbol{v} d x \quad \text { for all } \boldsymbol{v} \in W_{p, \text { div }}
\end{aligned}
$$

Consequently, the steady-state flow of Herschel-Bulkley fluid can be also described by the system (2-17)-(2-18). 


\section{Blockage property}

This section is dedicated to the study of the blockage property of Herschel-Bulkley fluid. To do this, let us recall the following standard definition [Hild et al. 2002].

Definition. We will say that the fluid is blocked in the domain $\Omega$ if $\boldsymbol{u}=0$ a.e. in $\Omega$ is a solution to the variational problem $\mathrm{P}_{g}$.

We prove the following proposition, which gives the variational interpretation of the blockage property.

Proposition. The fluid is blocked in the domain $\Omega$ if and only if

$$
g \int_{\Omega}|D(\boldsymbol{v})| d x \geq \int_{\Omega} \boldsymbol{f} \cdot \boldsymbol{v} d x \quad \text { for all } \boldsymbol{v} \in W_{p, \text { div }} .
$$

Proof. The first implication is an immediate consequence of the definition of blockage property. For the second one, we proceed as follows. Suppose that (3-1) holds. In particular, we have

$$
g \int_{\Omega}|D(\boldsymbol{u})| d x \geq \int_{\Omega} \boldsymbol{f} \cdot \boldsymbol{u} d x .
$$

Subtracting the inequalities (2-17) and (3-1), we find

$$
\begin{aligned}
\mu \int_{\Omega}|D(\boldsymbol{u})|^{p} d x \leq B(\boldsymbol{u} & , \boldsymbol{u}, \boldsymbol{v})+\mu \int_{\Omega}|D(\boldsymbol{u})|^{p-2} D(\boldsymbol{u}) \cdot D(\boldsymbol{v}) d x \\
& +g \int_{\Omega}|D(\boldsymbol{v})| d x-\int_{\Omega} \boldsymbol{f} \cdot \boldsymbol{v} d x \quad \text { for all } \boldsymbol{v} \in W_{p, \text { div }}
\end{aligned}
$$

Thus, the result can be obtained by setting $v=0$ as a test function in (3-3) and using Korn's inequality.

Hence, the mathematical study of the blockage property consists of finding the relationship between the yield limit $g$ and the density of volume forces $f$ such that the inequality (3-1) holds.

We say that $g$ is a blocking limit if the inequality (3-1) is satisfied.

We suppose from now on that

$$
f \in L^{\infty}(\Omega)^{n} .
$$

The statement below ensures the existence of a blocking phase for large-enough yield limit.

Proposition. If (3-4) holds, then

$$
g^{*}=\sup _{\boldsymbol{v} \in W_{p, \mathrm{div}}-\{0\}} \frac{\int_{\Omega} \boldsymbol{f} \cdot \boldsymbol{v} d x}{\int_{\Omega}|D(\boldsymbol{v})| d x}<+\infty .
$$

In addition, if $g \geq g^{*}$, then the blocking occurs; it means that (3-1) holds. 
Proof. Let us define the form $l \in W_{p \text {,div }}^{\prime}$ by

$$
\langle l, \boldsymbol{v}\rangle_{W_{p, \mathrm{div}}^{\prime} \times W_{p, \mathrm{div}}}=\int_{\Omega} \boldsymbol{f} \cdot \boldsymbol{v} d x \quad \text { for all } \boldsymbol{v} \in W_{p, \mathrm{div}} .
$$

The fact that $f \in L^{\infty}(\Omega)^{n}$ implies that $l \in W^{\prime}$. Then, there exists $C_{1}>0$ such that

$$
\left|\langle l, \boldsymbol{v}\rangle_{W_{p, \text { div }}^{\prime} \times W_{p, \text { div }}}\right| \leq C_{1}\|\boldsymbol{v}\|_{L D(\Omega)} \quad \text { for all } \boldsymbol{v} \in L D(\Omega) .
$$

This yields, thanks to the Korn inequality (2-13)

$$
\left|\langle l, \boldsymbol{v}\rangle_{W_{p, \text { div }}^{\prime}} \times W_{p, \text { div }}\right| \leq C_{1} C_{W}\|D(\boldsymbol{v})\|_{L^{1}(\Omega)^{n \times n}} \quad \text { for all } \boldsymbol{v} \in L D(\Omega) .
$$

Consequently, via (3-7) and (3-8) we obtain $g^{*} \leq C_{1} C_{W}$.

Now, if $g \geq g^{*}$, then (3-5) gives

$$
g \int_{\Omega}|D(\boldsymbol{v})| d x \geq g^{*} \int_{\Omega}|D(\boldsymbol{v})| d x \geq \int_{\Omega} \boldsymbol{f} \cdot \boldsymbol{v} d x \quad \text { for all } \boldsymbol{v} \in W_{p, \operatorname{div}}-\{0\},
$$

which completes the proof, by observing that if $v=0$, the inequality above also remains satisfied.

Here $g^{*}$ is said to be the minimal blocking limit.

Let $g$ be a blocking limit. We denote by $C$ the set

$$
C=\left\{\boldsymbol{v} \in W_{p, \operatorname{div}}\left|g \int_{\Omega}\right| D(\boldsymbol{v}) \mid d x=\int_{\Omega} \boldsymbol{f} \cdot \boldsymbol{v} d x\right\} .
$$

It is straightforward to verify that the set $C$ is a convex cone in $W_{p \text {,div }}$.

\section{Behaviour of the flow}

Let us introduce for $\varepsilon>0$ the perturbed yield limit

$$
g_{\varepsilon}=\left(1-\varepsilon^{p-1}\right) g
$$

and denote by $\boldsymbol{u}_{\varepsilon}$ the solution of the corresponding problem, i.e.,

$$
\begin{array}{r}
B\left(\boldsymbol{u}_{\varepsilon}, \boldsymbol{u}_{\varepsilon}, \boldsymbol{v}-\boldsymbol{u}_{\varepsilon}\right)+\mu \int_{\Omega}\left|D\left(\boldsymbol{u}_{\varepsilon}\right)\right|^{p-2} D\left(\boldsymbol{u}_{\varepsilon}\right) \cdot D\left(\boldsymbol{v}-\boldsymbol{u}_{\varepsilon}\right) d x+g_{\varepsilon} \int_{\Omega}|D(\boldsymbol{v})| d x \\
-g_{\varepsilon} \int_{\Omega}\left|D\left(\boldsymbol{u}_{\varepsilon}\right)\right| d x \geq \int_{\Omega} \boldsymbol{f} \cdot\left(\boldsymbol{v}-\boldsymbol{u}_{\varepsilon}\right) d x \quad \text { for all } \boldsymbol{v} \in W_{p, \text { div }} .
\end{array}
$$


The above inequality can be written in equivalent form

$$
\begin{aligned}
\mu \int_{\Omega}\left|D\left(\boldsymbol{u}_{\varepsilon}\right)\right|^{p} d x+g_{\varepsilon} \int_{\Omega}\left|D\left(\boldsymbol{u}_{\varepsilon}\right)\right| d x & =\int_{\Omega} \boldsymbol{f} \cdot \boldsymbol{u}_{\varepsilon} d x \\
B\left(\boldsymbol{u}_{\varepsilon}, \boldsymbol{u}_{\varepsilon}, \boldsymbol{v}\right)+\mu \int_{\Omega}\left|D\left(\boldsymbol{u}_{\varepsilon}\right)\right|^{p-2} D\left(\boldsymbol{u}_{\varepsilon}\right) \cdot D(\boldsymbol{v}) d x+g_{\varepsilon} \int_{\Omega}|D(\boldsymbol{v})| d x & \\
& \geq \int_{\Omega} \boldsymbol{f} \cdot \boldsymbol{v} d x \quad \text { for all } \boldsymbol{v} \in W_{p, \text { div }}
\end{aligned}
$$

Setting now

$$
\boldsymbol{w}_{\varepsilon}=\frac{\boldsymbol{u}_{\varepsilon}}{\varepsilon} \quad \text { for all } \varepsilon>0,
$$

in the following we establish a convergence result for $\left(\boldsymbol{w}_{\varepsilon}\right)_{\varepsilon>0}$ when $\varepsilon$ tends to 0 .

Theorem. Suppose that $g$ is a blocking limit. Then $\left(\boldsymbol{w}_{\varepsilon}\right)_{\varepsilon>0}$ convergences strongly, when $\varepsilon$ tends to 0 in $W_{p \text {, div }}$, to the solution $w$ of the variational inequality

$$
w \in C: \mu \int_{\Omega}|D(\boldsymbol{w})|^{p-2} D(\boldsymbol{w}) \cdot D(\boldsymbol{v}-\boldsymbol{w}) d x \geq \int_{\Omega} \boldsymbol{f} \cdot(\boldsymbol{v}-\boldsymbol{w}) d x \text { for all } \boldsymbol{v} \in C .
$$

Proof. The system becomes, taking into account (4-5),

$$
\begin{gathered}
\mu \varepsilon^{p-1} \int_{\Omega}\left|D\left(\boldsymbol{w}_{\varepsilon}\right)\right|^{p} d x+\left(1-\varepsilon^{p-1}\right) g \int_{\Omega}\left|D\left(\boldsymbol{w}_{\varepsilon}\right)\right| d x=\int_{\Omega} \boldsymbol{f} \cdot \boldsymbol{w}_{\varepsilon} d x, \\
\varepsilon^{2} B\left(\boldsymbol{w}_{\varepsilon}, \boldsymbol{w}_{\varepsilon}, \boldsymbol{v}\right)+\mu \varepsilon^{p-1} \int_{\Omega}\left|D\left(\boldsymbol{w}_{\varepsilon}\right)\right|^{p-2} D\left(\boldsymbol{w}_{\varepsilon}\right) \cdot D(\boldsymbol{v}) d x \\
+\left(1-\varepsilon^{p-1}\right) g \int_{\Omega}|D(\boldsymbol{v})| d x \geq \int_{\Omega} \boldsymbol{f} \cdot \boldsymbol{v} d x \quad \text { for all } \boldsymbol{v} \in W_{p, \text { div }}
\end{gathered}
$$

Equation (4-7) gives

$$
\begin{array}{r}
\mu \varepsilon^{p-1} \int_{\Omega}\left|D\left(\boldsymbol{w}_{\varepsilon}\right)\right|^{p} d x+\left(1-\varepsilon^{p-1}\right)\left(g \int_{\Omega}\left|D\left(\boldsymbol{w}_{\varepsilon}\right)\right| d x-\int_{\Omega} \boldsymbol{f} \cdot \boldsymbol{w}_{\varepsilon} d x\right) \\
=\varepsilon^{p-1} g \int_{\Omega}\left|D\left(\boldsymbol{w}_{\varepsilon}\right)\right| d x .
\end{array}
$$

Suppose that $g$ is a blocking limit; then (4-9) gives

$$
\mu \int_{\Omega}\left|D\left(\boldsymbol{w}_{\varepsilon}\right)\right|^{p} d x \leq g \int_{\Omega}\left|D\left(\boldsymbol{w}_{\varepsilon}\right)\right| d x .
$$

We deduce making use of Korn's inequality and some algebraic manipulations that

$$
\left\|\boldsymbol{w}_{\varepsilon}\right\|_{W_{p, \text { div }}} \leq c .
$$

Hence, we can extract a subsequence still denoted by $\left(\boldsymbol{w}_{\varepsilon}\right)_{\varepsilon>0}$ such that

$$
\boldsymbol{w}_{\varepsilon} \rightarrow \boldsymbol{w} \text { in } W_{p, \operatorname{div}} \text { weakly. }
$$


The Rellich-Kondrachov compactness theorem allows us to get after a new extraction

$$
\boldsymbol{w}_{\varepsilon} \rightarrow \boldsymbol{w} \quad \text { in } L^{p}(\Omega)^{n} \text { strongly and a.e. in } \Omega .
$$

Therefore, (4-9) gives again

$$
\left(1-\varepsilon^{p-1}\right) g \int_{\Omega}\left|D\left(\boldsymbol{w}_{\varepsilon}\right)\right| d x \leq \int_{\Omega} \boldsymbol{f} \cdot \boldsymbol{w}_{\varepsilon} d x
$$

thereby allowing us to find

$$
g \liminf \int_{\Omega}\left|D\left(\boldsymbol{w}_{\varepsilon}\right)\right| d x \leq \lim \int_{\Omega} \boldsymbol{f} \cdot \boldsymbol{w}_{\varepsilon} d x .
$$

This yields

$$
g \int_{\Omega}|D(\boldsymbol{w})| d x \leq \int_{\Omega} \boldsymbol{f} \cdot \boldsymbol{w} d x .
$$

Consequently, since $g$ is a blocking limit,

$$
g \int_{\Omega}|D(\boldsymbol{w})| d x=\int_{\Omega} \boldsymbol{f} \cdot \boldsymbol{w} d x .
$$

Taking $\boldsymbol{w}$ as test function in inequality (4-8), it implies that

$$
\begin{aligned}
\varepsilon^{2} B\left(\boldsymbol{w}_{\varepsilon}, \boldsymbol{w}_{\varepsilon}, \boldsymbol{w}\right)+\mu \varepsilon^{p-1} \int_{\Omega}\left|D\left(\boldsymbol{w}_{\varepsilon}\right)\right|^{p-2} D\left(\boldsymbol{w}_{\varepsilon}\right) \cdot D(\boldsymbol{w}) d x & \\
& +\left(1-\varepsilon^{p-1}\right) g \int_{\Omega}|D(\boldsymbol{w})| d x \geq \int_{\Omega} \boldsymbol{f} \cdot \boldsymbol{w} d x .
\end{aligned}
$$

This gives, making use of (4-16),

$$
\varepsilon^{3-p} B\left(\boldsymbol{w}_{\varepsilon}, \boldsymbol{w}_{\varepsilon}, \boldsymbol{w}\right)+\mu \int_{\Omega}\left|D\left(\boldsymbol{w}_{\varepsilon}\right)\right|^{p-2} D\left(\boldsymbol{w}_{\varepsilon}\right) \cdot D(\boldsymbol{w}) d x \geq g \int_{\Omega}|D(\boldsymbol{w})| d x .
$$

Moreover, the lemma on page 66 permits us to obtain the estimate

$$
\left|B\left(\boldsymbol{w}_{\varepsilon}, \boldsymbol{w}_{\varepsilon}, \boldsymbol{w}\right)\right| \leq\left\|\boldsymbol{w}_{\varepsilon}\right\|_{W_{p, \text { div }}}^{2}\|\boldsymbol{w}\|_{W_{p, \text { div }}} .
$$

On the other hand, it is well known that the nonlinear term $\mu \int_{\Omega}\left|D\left(\boldsymbol{w}_{\varepsilon}\right)\right|^{p-2} D\left(\boldsymbol{w}_{\varepsilon}\right)$. $D(\boldsymbol{w}) d x$ converges to $\mu \int_{\Omega}|D(\boldsymbol{w})|^{p} d x$ [Lions 1969]. Consequently, by passing to the limit, one can find, keeping in mind (4-18),

$$
\mu \int_{\Omega}|D(\boldsymbol{w})|^{p} d x \geq g \int_{\Omega}|D(\boldsymbol{w})| d x .
$$

We get thanks to (4-10)

$$
\liminf \mu \int_{\Omega}\left|D\left(\boldsymbol{w}_{\varepsilon}\right)\right|^{p} d x \leq g \liminf \int_{\Omega}\left|D\left(\boldsymbol{w}_{\varepsilon}\right)\right| d x .
$$


So, using (4-14) we can infer that

$$
\liminf \mu \int_{\Omega}\left|D\left(\boldsymbol{w}_{\varepsilon}\right)\right|^{p} d x \leq \lim \int_{\Omega} \boldsymbol{f} \cdot \boldsymbol{w}_{\varepsilon} d x,
$$

which implies that

$$
\mu \int_{\Omega}|D(\boldsymbol{w})|^{p} d x \leq \int_{\Omega} \boldsymbol{f} \cdot \boldsymbol{w} d x
$$

Putting together (4-16), (4-19), and (4-20) we obtain

$$
\mu \int_{\Omega}|D(\boldsymbol{w})|^{p} d x=g \int_{\Omega}|D(\boldsymbol{w})| d x=\int_{\Omega} \boldsymbol{f} \cdot \boldsymbol{w} d x
$$

which implies in particular that $\boldsymbol{w} \in C$. Furthermore, by (4-8) we get

$$
\begin{array}{rl}
\varepsilon^{3-p} & B\left(\boldsymbol{w}_{\varepsilon}, \boldsymbol{w}_{\varepsilon}, \boldsymbol{v}\right)+\mu \int_{\Omega}\left|D\left(\boldsymbol{w}_{\varepsilon}\right)\right|^{p-2} D\left(\boldsymbol{w}_{\varepsilon}\right) \cdot D(\boldsymbol{v}) d x \\
& +\frac{1}{\varepsilon^{p-1}}\left(g \int_{\Omega}|D(\boldsymbol{v})| d x-\int_{\Omega} \boldsymbol{f} \cdot \boldsymbol{v} d x\right) \geq g \int_{\Omega}|D(\boldsymbol{v})| d x \quad \text { for all } \boldsymbol{v} \in W_{p, \operatorname{div} .}
\end{array}
$$

By choosing $v \in C$ in the above inequality, the passage to the limit leads to

$$
\mu \int_{\Omega}|D(\boldsymbol{w})|^{p-2} D(\boldsymbol{w}) \cdot D(\boldsymbol{v}) d x \geq g \int_{\Omega}|D(\boldsymbol{v})| d x \quad \text { for all } \boldsymbol{v} \in C .
$$

This yields

$$
\mu \int_{\Omega}|D(\boldsymbol{w})|^{p-2} D(\boldsymbol{w}) \cdot D(\boldsymbol{v}) d x \geq \int_{\Omega} \boldsymbol{f} \cdot \boldsymbol{v} d x \quad \text { for all } \boldsymbol{v} \in C .
$$

Combining (4-21) and (4-22) yields the inequality (4-6).

Our objective now is to prove the strong convergence. With this aim, we proceed as follows. The use of (4-7) and (4-8) permits us to affirm that for every $\boldsymbol{v} \in W_{p \text {, div }}$

$$
\begin{aligned}
\varepsilon^{2} B\left(\boldsymbol{w}_{\varepsilon}, \boldsymbol{w}_{\varepsilon}, \boldsymbol{v}\right)+\mu \varepsilon^{p-1} \int_{\Omega}\left|D\left(\boldsymbol{w}_{\varepsilon}\right)\right|^{p-2} D\left(\boldsymbol{w}_{\varepsilon}\right) \cdot D\left(\boldsymbol{v}-\boldsymbol{w}_{\varepsilon}\right) d x \\
\geq \int_{\Omega} \boldsymbol{f} \cdot\left(\boldsymbol{v}-\boldsymbol{w}_{\varepsilon}\right) d x-\left(1-\varepsilon^{p-1}\right) g\left(\int_{\Omega}|D(\boldsymbol{v})| d x-\int_{\Omega}\left|D\left(\boldsymbol{w}_{\varepsilon}\right)\right| d x\right)
\end{aligned}
$$

It follows, by setting $\boldsymbol{v}=\boldsymbol{w}$, that

$$
\begin{aligned}
-\varepsilon^{2} B\left(\boldsymbol{w}_{\varepsilon}, \boldsymbol{w}_{\varepsilon}, \boldsymbol{w}\right)+\mu \varepsilon^{p-1} \int_{\Omega}\left|D\left(\boldsymbol{w}_{\varepsilon}\right)\right|^{p-2} D\left(\boldsymbol{w}_{\varepsilon}\right) \cdot D\left(\boldsymbol{w}_{\varepsilon}-\boldsymbol{w}\right) d x \\
\leq \int_{\Omega} \boldsymbol{f} \cdot\left(\boldsymbol{w}_{\varepsilon}-\boldsymbol{w}\right) d x+\left(1-\varepsilon^{p-1}\right) g \int_{\Omega}\left(|D(\boldsymbol{w})|-\left|D\left(\boldsymbol{w}_{\varepsilon}\right)\right|\right) d x .
\end{aligned}
$$

Further, since $g$ is the blocking limit and $\boldsymbol{w} \in W_{p \text {, div }}$, one can verify that

$$
g \int_{\Omega}\left(|D(\boldsymbol{w})|-\left|D\left(\boldsymbol{w}_{\varepsilon}\right)\right|\right) d x \leq \int_{\Omega} \boldsymbol{f} \cdot\left(\boldsymbol{w}-\boldsymbol{w}_{\varepsilon}\right) d x .
$$


Consequently, inequality (4-23) becomes

$\mu \int_{\Omega}\left|D\left(\boldsymbol{w}_{\varepsilon}\right)\right|^{p-2} D\left(\boldsymbol{w}_{\varepsilon}\right) \cdot D\left(\boldsymbol{w}_{\varepsilon}-\boldsymbol{w}\right) d x \leq \int_{\Omega} \boldsymbol{f} \cdot\left(\boldsymbol{w}_{\varepsilon}-\boldsymbol{w}\right) d x+\varepsilon^{3-p} B\left(\boldsymbol{w}_{\varepsilon}, \boldsymbol{w}_{\varepsilon}, \boldsymbol{w}\right)$.

This becomes

$$
\begin{aligned}
& \mu \int_{\Omega}\left(\left|D\left(\boldsymbol{w}_{\varepsilon}\right)\right|^{p-2} D\left(\boldsymbol{w}_{\varepsilon}\right)-|D(\boldsymbol{w})|^{p-2} D(\boldsymbol{w})\right) D\left(\boldsymbol{w}_{\varepsilon}-\boldsymbol{w}\right) d x \\
& \leq \int_{\Omega} \boldsymbol{f} \cdot\left(\boldsymbol{w}_{\varepsilon}-\boldsymbol{w}\right) d x-\mu \int_{\Omega}|D(\boldsymbol{w})|^{p-2} D(\boldsymbol{w}) D\left(\boldsymbol{w}_{\varepsilon}-\boldsymbol{w}\right) d x \\
& \quad+c \varepsilon^{3-p}\left\|\boldsymbol{w}_{\varepsilon}\right\|_{W_{p, \text { div }}}^{2}\|\boldsymbol{w}\|_{W_{p, \mathrm{div}}} .
\end{aligned}
$$

Let us observe now that for every $x, y \in \mathbb{R}^{n}$,

$$
\left(|x|^{p-2} x-|y|^{p-2} y\right) \cdot(x-y) \geq c \frac{|x-y|^{2}}{(|x|+|y|)^{2-p}}, \quad 1<p \leq 2 .
$$

So applying the above inequality, (4-25) can be rewritten as

$$
\begin{aligned}
& \mu \int_{\Omega} \frac{\left|D\left(\boldsymbol{w}_{\varepsilon}-\boldsymbol{w}\right)\right|^{2}}{\left(\left|D\left(\boldsymbol{w}_{\varepsilon}\right)\right|+|D(\boldsymbol{w})|\right)^{2-p}} d x \leq c\left|\int_{\Omega} \boldsymbol{f} \cdot\left(\boldsymbol{w}_{\varepsilon}-\boldsymbol{w}\right) d x\right| \\
& \quad+c \varepsilon^{3-p}\left\|\boldsymbol{w}_{\varepsilon}\right\|_{W_{p, \text { div }}}^{2}\|\boldsymbol{w}\|_{W_{p, \text { div }}}+\left.c \mu\left|\int_{\Omega}\right| D(\boldsymbol{w})\right|^{p-2} D(\boldsymbol{w}) D\left(\boldsymbol{w}_{\varepsilon}-\boldsymbol{w}\right) d x \mid,
\end{aligned}
$$

which gives, exploiting Korn's and Hölder's inequalities,

$$
\begin{aligned}
\left\|\boldsymbol{w}_{\varepsilon}-\boldsymbol{w}\right\|_{W_{p}}^{p} & \leq c\left(\int_{\Omega}\left(\left|D\left(\boldsymbol{w}_{m}\right)\right|+|D(\boldsymbol{w})|\right)^{p} d x\right)^{(2-p) / 2}\left(\left|\int_{\Omega} \boldsymbol{f} \cdot\left(\boldsymbol{w}_{\varepsilon}-\boldsymbol{w}\right) d x\right|\right. \\
& \left.+\varepsilon^{3-p}\left\|\boldsymbol{w}_{\varepsilon}\right\|_{W_{p, \text { div }}}^{2}\|\boldsymbol{w}\|_{W_{p, \text { div }}}+\mu \int_{\Omega}|D(\boldsymbol{w})|^{p-2} D(\boldsymbol{w}) D\left(\boldsymbol{w}_{\varepsilon}-\boldsymbol{w}\right) d x\right)^{p / 2} .
\end{aligned}
$$

Passing to the limit, we conclude, using (4-12) and taking into account the fact that $|D(\boldsymbol{w})|^{p-2} D(\boldsymbol{w})$ is bounded in $L^{p^{\prime}}(\Omega)^{n}$, that

$$
\boldsymbol{w}_{\varepsilon} \rightarrow \boldsymbol{w} \text { in } W_{p, \text { div }} \text { strongly, }
$$

which permits us to complete the proof.

Corollary. Denoting by $\boldsymbol{w}_{0}$ the unique solution of the variational equation given by

$$
\mu \int_{\Omega}\left|D\left(\boldsymbol{w}_{0}\right)\right|^{p-2} D\left(\boldsymbol{w}_{0}\right) \cdot D(\boldsymbol{v}) d x=\int_{\Omega} \boldsymbol{f} \cdot \boldsymbol{v} d x \quad \text { for all } \boldsymbol{v} \in W_{p, \mathrm{div}},
$$

then the following estimates hold:

$$
\|D(\boldsymbol{w})\|_{L^{p}(\Omega)^{n \times n}} \leq\left\|D\left(\boldsymbol{w}_{0}\right)\right\|_{L^{p}(\Omega)^{n \times n}}, \quad \int_{\Omega} \boldsymbol{f} \cdot \boldsymbol{w} d x \leq \int_{\Omega} \boldsymbol{f} \cdot \boldsymbol{w}_{0} d x .
$$


Proof. We can infer by setting $\boldsymbol{w}$ as a test function in (4-26) that

$$
\mu \int_{\Omega}\left|D\left(\boldsymbol{w}_{0}\right)\right|^{p-2} D\left(\boldsymbol{w}_{0}\right) \cdot D(\boldsymbol{w}) d x=\int_{\Omega} \boldsymbol{f} \cdot \boldsymbol{w} d x .
$$

This yields, using Hölder's inequality

$$
\mu\left\|D\left(\boldsymbol{w}_{0}\right)\right\|_{L^{p}(\Omega)^{n \times n}}^{p-1}\|D(\boldsymbol{w})\|_{L^{p}(\Omega)^{n \times n}} \geq \int_{\Omega} \boldsymbol{f} \cdot \boldsymbol{w} d x=\mu\|D(\boldsymbol{w})\|_{L^{p}(\Omega)^{n \times n}}^{p},
$$

which allows us to get the first estimate. The second estimate becomes an immediate consequence of the first one.

\section{References}

[Frehse et al. 2003] J. Frehse, J. Málek, and M. Steinhauer, "On analysis of steady flows of fluids with shear-dependent viscosity based on the Lipschitz truncation method", SIAM J. Math. Anal. 34:5 (2003), 1064-1083.

[Hild et al. 2002] P. Hild, I. R. Ionescu, T. Lachand-Robert, and I. Roşca, "The blocking of an inhomogeneous Bingham fluid: applications to landslides", M2AN Math. Model. Numer. Anal. 36:6 (2002), 1013-1026.

[Lions 1969] J.-L. Lions, Quelques méthodes de résolution des problèmes aux limites non linéaires, Dunod, Paris, 1969.

[Málek 2008] J. Málek, "Mathematical properties of flows of incompressible power-law-like fluids that are described by implicit constitutive relations", Electron. Trans. Numer. Anal. 31 (2008), 110125.

[Málek et al. 2005] J. Málek, M. Růžička, and V. V. Shelukhin, "Herschel-Bulkley fluids: existence and regularity of steady flows", Math. Models Methods Appl. Sci. 15:12 (2005), 1845-1861.

[Málek et al. 2006] J. Málek, D. Pražák, and M. Steinhauer, "On the existence and regularity of solutions for degenerate power-law fluids", Differ. Integral Equ. 19:4 (2006), 449-462.

[Messelmi 2017] F. Messelmi, "Asymptotic behavior of unsteady Herschel-Bulkley flow", An. Univ. Oradea Fasc. Mat. 24:2 (2017), 181-194.

[Messelmi and Merouani 2010] F. Messelmi and B. Merouani, "Stationary thermal flow of a Bingham fluid whose viscosity, yield limit and friction depend on the temperature", An. Univ. Oradea Fasc. Mat. 17:2 (2010), 59-74.

[Messelmi and Merouani 2013] F. Messelmi and A. Merouani, "Properties of the laminar flow of Herschel-Bulkley fluid”, An. Univ. Oradea Fasc. Mat. 20:1 (2013), 47-60.

[Messelmi et al. 2010] F. Messelmi, B. Merouani, and F. Bouzeghaya, "Steady-state thermal HerschelBulkley flow with Tresca's friction law”, Electron. J. Differential Equations 2010:46 (2010).

[Temam 1985] R. Temam, Mathematical problems in plasticity, Mathematical Methods of Information Science 12, Gauthier-Villars, Paris, 1985.

Received 2 Sep 2018. Revised 10 Nov 2018. Accepted 11 Dec 2018.

FARID MESSELMI: foudimath@yahoo.fr

Department of Mathematics and LDMM Laboratory, University Ziane Achour of Djelfa, Djelfa, Algeria 



\title{
CONTINUUM THEORY FOR MECHANICAL METAMATERIALS WITH A CUBIC LATTICE SUBSTRUCTURE
}

\author{
Simon R. Eugster, Francesco dell' Isola And David J. Steigmann
}

\begin{abstract}
A three-dimensional continuum theory for fibrous mechanical metamaterials is proposed, in which the fibers are assumed to be spatial Kirchhoff rods whose mechanical response is controlled by a deformation field and a rotation field, the former accounting for strain of the rod and the latter for flexure and twist of the rod as it deforms. This leads naturally to a model based on Cosserat elasticity. Rigidity constraints are introduced that effectively reduce the model to a variant of second-gradient elasticity theory.
\end{abstract}

\section{Introduction}

The advent of 3D printing and associated microfabrication technologies has facilitated the design and realization of a range of mechanical metamaterials. These lightweight artificial materials exhibit stiffness and energy-absorbing properties far exceeding those of conventional bulk materials [Barchiesi et al. 2019; Mieszala et al. 2017; Vangelatos et al. 2019]. A unit cell of such a material - typically of microscopic dimensions - consists of a lattice of beam-like or rod-like fibers interacting at internal connections. The intrinsic extensional, flexural, and torsional stiffnesses of the fibers combine with the architecture of the lattice to confer high stiffness on the material at the macroscale together with enhanced energy absorption via microscale buckling.

These technologies provide impetus for the development of a continuum theory for the analysis of the macroscale response of metamaterials with lattice-like substructures. Toward this end, we outline a Cosserat model in which the deformation and rotation fields account respectively for the strains and orientations of the fibers, regarded as spatial Kirchhoff rods. In this preliminary work we confine attention to the simplest case of a cubic lattice architecture in which the constituent fibers are

\section{Communicated by Holm Altenbach.}

This work was initiated while Eugster and dell'Isola held visiting appointments at the University of California, Berkeley. Steigmann gratefully acknowledges the support of the U.S. National Science Foundation through grant CMMI 1538228.

MSC2010: 74A05, 74A30, 74A60, 74B20.

Keywords: mechanical metamaterials, Cosserat elasticity, strain-gradient theory. 
initially orthogonal. Further, in view of the solid nature of the internal connections in typical metamaterials [Vangelatos et al. 2019], we assume the fibers to be rigid in the sense that they remain mutually orthogonal in the course of deformation. They are free to extend or contract and to bend and twist, however, and these modes of deformation are modeled explicitly.

In Section 2 we review Kirchhoff's one-dimensional theory of rods as a prelude to the development, in Section 3, of an analogous three-dimensional Cosserat continuum model. We show that the rigidity constraint imposed in the continuum theory effectively determines the Cosserat rotation in terms of the gradient of the deformation field. Because the gradient of the rotation field is involved in the constitutive functions, this has the consequence that the Cosserat model reduces to a particular second-gradient theory of elasticity [Spencer and Soldatos 2007]. Details of this reduction are given in Section 4. Finally, in Section 5 we apply the theory to predict the response of a block to finite flexure. This solution serves to illustrate certain unusual features of the proposed model.

\section{Kirchoff rods}

In Kirchhoff's theory the rod is regarded as a spatial curve endowed with an elastic strain-energy function that depends on curvature and twist [Landau and Lifshitz 1986; Dill 1992; Antman 2005]. In Dill's derivation from conventional threedimensional nonlinear elasticity, this theory also accommodates small axial strain along the rod, whereas this effect is suppressed in derivations based on asymptotic analysis or the method of gamma convergence. We include it here. In the present section we outline the basic elements of Kirchhoff's theory, including the kinematics, the constitutive theory, and the variational derivation of the equilibrium equations. Although this theory is well known, we review it here to facilitate the interpretation of the ensuing continuum theory of metamaterials.

2.1. Kinematics. The basic kinematic variables in the theory are a deformation field $\boldsymbol{r}(s)$, where $s \in[0, l]$ and $l$ is the length of the rod in a reference configuration, and a right-handed, orthonormal triad $\left\{\boldsymbol{d}_{i}(s)\right\}$ in which $\boldsymbol{d}_{3}=\boldsymbol{d}$, where $\boldsymbol{d}$ is the unit vector defined by [Dill 1992; Steigmann 1996; Antman 2005]

$$
\begin{aligned}
& \boldsymbol{r}^{\prime}(s)=\lambda \boldsymbol{d}, \\
& \lambda=\left|\boldsymbol{r}^{\prime}(s)\right| .
\end{aligned}
$$

Here $\lambda$ is the stretch of the rod. Thus, $\boldsymbol{d}$ is the unit tangent to the rod in a deformed configuration and $\boldsymbol{d}_{\alpha}(\alpha=1,2)$ span its cross-sectional plane at arclength station $s$.

A central aspect of Kirchhoff's theory is that each cross section deforms as a rigid disc. Accordingly, there is a rotation field $\boldsymbol{R}(s)$ such that $\boldsymbol{d}_{i}=\boldsymbol{R} \boldsymbol{D}_{i}$, where 
$\boldsymbol{D}_{i}(s)$ are the values of $\boldsymbol{d}_{i}(s)$ in a reference configuration; thus,

$$
\boldsymbol{R}=\boldsymbol{d}_{i} \otimes \boldsymbol{D}_{i}
$$

The curvature and twist of the rod are computed from the derivatives $\boldsymbol{d}_{i}^{\prime}(s)$, where

$$
\boldsymbol{d}_{i}^{\prime}=\boldsymbol{R}^{\prime} \boldsymbol{D}_{i}+\boldsymbol{R} \boldsymbol{D}_{i}^{\prime}
$$

Let $\left\{\boldsymbol{E}_{i}\right\}$ be a fixed right-handed background frame. Then $\boldsymbol{D}_{i}(s)=\boldsymbol{A}(s) \boldsymbol{E}_{i}$ for some rotation field $\boldsymbol{A}$, yielding

$$
\boldsymbol{d}_{i}^{\prime}=\boldsymbol{W} \boldsymbol{d}_{i}=\boldsymbol{w} \times \boldsymbol{d}_{i},
$$

where

$$
\boldsymbol{W}=\boldsymbol{R}^{\prime} \boldsymbol{R}^{t}+\boldsymbol{R} \boldsymbol{A}^{\prime} \boldsymbol{A}^{t} \boldsymbol{R}^{t}
$$

is a skew tensor and $\boldsymbol{w}$ is its axial vector. If the rod is straight and untwisted in the reference configuration, i.e., if $\boldsymbol{D}_{i}^{\prime}=\mathbf{0}$, then $\boldsymbol{W}=\boldsymbol{R}^{\prime} \boldsymbol{R}^{t}$.

2.2. Strain-energy function. We assume the strain energy $E$ stored in a segment $\left[l_{1}, l_{2}\right] \subset[0, l]$ of a rod of length $l$ to be expressible as

$$
E=\int_{l_{1}}^{l_{2}} U d s
$$

where $U$, the strain energy per unit length, is a function of the list $\left\{\boldsymbol{R}, \boldsymbol{R}^{\prime}, \boldsymbol{r}^{\prime}\right\}$, possibly depending explicitly on $s$. Explicit $s$-dependence may arise from the initial curvature or twist of the rod, or from nonuniform material properties.

We require $U$ to be Galilean invariant and hence that its values be unaffected by the substitution $\left\{\boldsymbol{R}, \boldsymbol{R}^{\prime}, \boldsymbol{r}^{\prime}\right\} \rightarrow\left\{\boldsymbol{Q R}, \boldsymbol{Q} \boldsymbol{R}^{\prime}, \boldsymbol{Q} \boldsymbol{r}^{\prime}\right\}$, where $\boldsymbol{Q}$ is an arbitrary uniform rotation. Because $U$ is defined pointwise, to derive a necessary condition we select the rotation $\boldsymbol{Q}=\boldsymbol{R}_{\mid s}^{t}$ and conclude that $U$ is determined by the list $\left\{\boldsymbol{R}^{t} \boldsymbol{R}^{\prime}, \boldsymbol{R}^{t} \boldsymbol{r}^{\prime}\right\}$. This list is trivially Galilean invariant. It is equivalent to $\left\{\boldsymbol{R}^{t} \boldsymbol{W} \boldsymbol{R}-\boldsymbol{A}^{\prime} \boldsymbol{A}^{t}, \lambda \boldsymbol{D}\right\}$, where $\boldsymbol{D}=\boldsymbol{D}_{3}$ and $\boldsymbol{R}^{t} \boldsymbol{W} \boldsymbol{R}-\boldsymbol{A}^{\prime} \boldsymbol{A}^{t}$ is a Galilean-invariant measure of the relative flexure and twist of the rod due to deformation. Here $\boldsymbol{D}$ and $\boldsymbol{A}^{\prime} \boldsymbol{A}^{t}$ are independent of the deformation and serve to confer an explicit $s$-dependence on the strainenergy function; accordingly, we write $U=U\left(\boldsymbol{R}^{t} \boldsymbol{W} \boldsymbol{R}, \lambda ; s\right)$. If the rod is initially straight and untwisted, as we assume hereafter, then $\boldsymbol{D}_{i}^{\prime}=\mathbf{0}$ and any explicit $s$ dependence of the energy is due solely to nonuniformity of the material properties. Henceforth, we assume material properties to be uniform.

In the present circumstances we have

$$
\boldsymbol{R}^{t} \boldsymbol{W} \boldsymbol{R}=W_{i j} \boldsymbol{D}_{i} \otimes \boldsymbol{D}_{j}, \quad \text { with } W_{i j}=\boldsymbol{d}_{i} \cdot \boldsymbol{W} \boldsymbol{d}_{j}=\boldsymbol{d}_{i} \cdot \boldsymbol{d}_{j}^{\prime} .
$$

Thus,

$$
U=W(\lambda, \kappa)
$$


where $\boldsymbol{\kappa}\left(=\kappa_{i} \boldsymbol{D}_{i}\right)$ is the axial vector of $\boldsymbol{R}^{t} \boldsymbol{W} \boldsymbol{R}$; i.e.,

$$
\kappa_{i}=\frac{1}{2} e_{i j k} \boldsymbol{d}_{k} \cdot \boldsymbol{d}_{j}^{\prime} \text {. }
$$

Here $e_{i j k}$ is the Levi-Civita permutation symbol $\left(e_{123}=+1\right.$, etc. $), \kappa_{3}$ is the twist of the rod, and $\kappa_{\alpha}$ are the curvatures. Moreover, it follows easily from (4) and (9) that

$$
\boldsymbol{w}=\boldsymbol{R} \boldsymbol{\kappa}=\kappa_{i} \boldsymbol{d}_{i}
$$

if the rod is initially straight and untwisted.

For example, in the classical theory [Dill 1992; Steigmann 1996; Antman 2005], the strain-energy function is

$$
W(\lambda, \kappa)=\frac{1}{2} E(\lambda-1)^{2}+\frac{1}{2} F \kappa_{\alpha} \kappa_{\alpha}+\frac{1}{2} T \tau^{2},
$$

where $\tau\left(=\kappa_{3}\right)$ is the twist, $E$ is the extensional stiffness (Young's modulus times the cross-sectional area), $F$ is the flexural stiffness (Young's modulus times the second moment of area of the cross section), and $T$ is the torsional stiffness (the shear modulus times the polar moment of the cross section).

The terms involving curvature and twist in this expression are appropriate for rods of circular cross section composed of isotropic materials [Landau and Lifshitz 1986]. The homogeneous quadratic dependence of the energy on these terms may be understood in terms of a local length scale such as the diameter of a fiber cross section. The curvature-twist vector, when nondimensionalized by this local scale, is typically small in applications. For example, the minimum radius of curvature of a bent fiber is typically much larger than the fiber diameter. If the bending and twisting moments vanish when the rod is straight and untwisted, then the leadingorder contribution of the curvature-twist vector to the strain energy is quadratic; this is reflected in (11). In general the flexural and torsional stiffnesses in this expression may depend on fiber stretch, but in the small-extensional-strain regime contemplated here, they are approximated at leading order by constants in the case of a uniform rod.

2.3. Variational theory. The equilibrium equations of the Kirchhoff theory are well known and easily derived from elementary considerations, but it is instructive to review their variational derivation here as a prelude to the considerations that follow.

We assume that equilibria of the rod are such as to satisfy the virtual-power statement

$$
\dot{E}=P \text {, }
$$

where $P$ is the virtual power of the loads - the explicit form of which is deduced below - and the superposed dot is used to identify a variational derivative. These 
are derivatives, with respect to $\epsilon$, of the one-parameter deformation and rotation fields $\boldsymbol{r}(s ; \epsilon)$ and $\boldsymbol{R}(s ; \epsilon)$, respectively, where $\boldsymbol{r}(s)=\boldsymbol{r}(s ; 0)$ and $\boldsymbol{R}(s)=\boldsymbol{R}(s ; 0)$ are equilibrium fields, and (see (11))

$$
\dot{U}=\dot{W}=W_{\lambda} \dot{\lambda}+\mu_{i} \dot{\kappa}_{i}
$$

where

$$
W_{\lambda}=\frac{\partial W}{\partial \lambda} \quad \text { and } \quad \mu_{i}=\frac{\partial W}{\partial \kappa_{i}}
$$

are evaluated at equilibrium, corresponding to $\epsilon=0$.

From (1) we have

$$
\dot{\lambda} d+\omega \times r^{\prime}=u^{\prime},
$$

where $\boldsymbol{u}(s)=\dot{\boldsymbol{r}}$ is the virtual translational velocity and $\boldsymbol{\omega}(s)$ is the axial vector of the skew tensor $\dot{\boldsymbol{R}} \boldsymbol{R}^{t}$; i.e.,

$$
\dot{d}_{i}=\omega \times d_{i}
$$

It follows from (9) and (16) that

$$
\begin{aligned}
\dot{\kappa}_{i} & =\frac{1}{2} e_{i j k}\left(\dot{\boldsymbol{d}}_{k} \cdot \boldsymbol{d}_{j}^{\prime}+\boldsymbol{d}_{k} \cdot \dot{\boldsymbol{d}}_{j}^{\prime}\right) \\
& =\frac{1}{2} e_{i j k}\left[\boldsymbol{\omega} \times \boldsymbol{d}_{k} \cdot \boldsymbol{d}_{j}^{\prime}+\boldsymbol{d}_{k} \cdot\left(\boldsymbol{\omega}^{\prime} \times \boldsymbol{d}_{j}+\boldsymbol{\omega} \times \boldsymbol{d}_{j}^{\prime}\right)\right],
\end{aligned}
$$

in which the terms involving $\omega$ cancel; the $e-\delta$ identity $\frac{1}{2} e_{i j k} e_{m j k}=\delta_{i m}$ (the Kronecker delta), combined with $\boldsymbol{d}_{j} \times \boldsymbol{d}_{k}=e_{m j k} \boldsymbol{d}_{m}$, then yields

$$
\dot{\kappa}_{i}=d_{i} \cdot \omega^{\prime}
$$

Thus,

$$
\dot{E}=I[\boldsymbol{u}, \boldsymbol{\omega}],
$$

where

$$
I[\boldsymbol{u}, \boldsymbol{\omega}]=\int_{l_{1}}^{l_{2}}\left(W_{\lambda} \boldsymbol{d} \cdot \boldsymbol{u}^{\prime}+\boldsymbol{\mu} \cdot \boldsymbol{\omega}^{\prime}\right) d s
$$

with

$$
\boldsymbol{\mu}=\mu_{i} \boldsymbol{d}_{i} .
$$

Further, from (1) we have the orthogonality constraints

$$
\boldsymbol{r}^{\prime} \cdot \boldsymbol{d}_{\alpha}=0
$$

for $\alpha=1,2$. To accommodate these in the variational formulation, we introduce the energy

$$
E^{*}=E+\int_{l_{1}}^{l_{2}} f_{\alpha} \boldsymbol{r}^{\prime} \cdot \boldsymbol{d}_{\alpha} d s
$$


where $f_{\alpha}(s)$ are Lagrange multipliers. This is an extension to arbitrary deformations of the actual energy, the latter being defined only for the class of deformations defined by the constraints. Moreover, (12) is replaced by

$$
\dot{E}^{*}=P \text {, }
$$

where

$$
\dot{E}^{*}=\int_{l_{1}}^{l_{2}}\left[\left(W_{\lambda} \boldsymbol{d}+f_{\alpha} \boldsymbol{d}_{\alpha}\right) \cdot \boldsymbol{u}^{\prime}+\boldsymbol{\mu} \cdot \boldsymbol{\omega}^{\prime}+f_{\alpha} \boldsymbol{d}_{\alpha} \times \boldsymbol{r}^{\prime} \cdot \boldsymbol{\omega}\right] d s .
$$

We do not make variations with respect to the multipliers $f_{\alpha}$ explicit as these merely return the constraints (22).

We conclude that $(24)$ reduces to

$$
\left.(\boldsymbol{f} \cdot \boldsymbol{u}+\boldsymbol{\mu} \cdot \boldsymbol{\omega})\right|_{l_{1}} ^{l_{2}}-\int_{l_{1}}^{l_{2}}\left[\boldsymbol{u} \cdot \boldsymbol{f}^{\prime}+\boldsymbol{\omega} \cdot\left(\boldsymbol{\mu}^{\prime}-\boldsymbol{f} \times \boldsymbol{r}^{\prime}\right)\right] d s=P,
$$

where

$$
\boldsymbol{f}=W_{\lambda} \boldsymbol{d}+f_{\alpha} \boldsymbol{d}_{\alpha} .
$$

This implies that the virtual power is expressible in the form

$$
P=\left.(\boldsymbol{t} \cdot \boldsymbol{u}+\boldsymbol{c} \cdot \boldsymbol{\omega})\right|_{l_{1}} ^{l_{2}}+\int_{l_{1}}^{l_{2}}(\boldsymbol{u} \cdot \boldsymbol{g}+\boldsymbol{\omega} \cdot \boldsymbol{\pi}) d s,
$$

in which $\boldsymbol{t}$ and $\boldsymbol{c}$ represent forces and couples acting at the ends of the segment and $\boldsymbol{g}$ and $\boldsymbol{\pi}$ are force and couple distributions acting in the interior.

By the fundamental lemma, the Euler equations holding at points in the interior of the rod are

$$
\boldsymbol{f}^{\prime}+\boldsymbol{g}=\mathbf{0} \quad \text { and } \quad \boldsymbol{\mu}^{\prime}+\boldsymbol{\pi}=\boldsymbol{f} \times \boldsymbol{r}^{\prime},
$$

and the endpoint conditions are

$$
f=t \quad \text { and } \quad \mu=c,
$$

provided that neither position nor section orientation is assigned at the endpoints. These are the equilibrium conditions of classical rod theory in which $\boldsymbol{f}$ and $\boldsymbol{\mu}$ respectively are the cross-sectional force and moment transmitted by the segment $(s, l]$ on the part $[0, s]$. Equations (27) and $(30)_{1}$ justify the interpretation of the Lagrange multipliers $f_{\alpha}$ as transverse shear forces acting on a fiber cross section.

Other boundary conditions are, of course, feasible. For example, if the tangent direction $\boldsymbol{d}$ is assigned at a boundary point, then its variation $\boldsymbol{\omega} \times \boldsymbol{d}$ vanishes there, leaving $\boldsymbol{\omega}=\omega \boldsymbol{d}$ in which $\omega$ is arbitrary. In this case (26) and (28) furnish the boundary condition

$$
\boldsymbol{\mu} \cdot \boldsymbol{d}=c,
$$

in which $c=\boldsymbol{c} \cdot \boldsymbol{d}$ is the twisting moment applied at the boundary. 
For the strain-energy function (11), we have

$$
W_{\lambda}=E(\lambda-1),
$$

together with $\mu_{3} \boldsymbol{d}_{3}=T \tau \boldsymbol{d}$ and $\mu_{\alpha} \boldsymbol{d}_{\alpha}=F \kappa_{\alpha} \boldsymbol{d}_{\alpha}$. To reduce the second expression we use (9), together with $\boldsymbol{d} \cdot \boldsymbol{d}_{\mu}^{\prime}=-\boldsymbol{d}_{\mu} \cdot \boldsymbol{d}^{\prime}$, to derive $\kappa_{\alpha}=e_{\alpha 3 \mu} \boldsymbol{d}_{\mu} \cdot \boldsymbol{d}^{\prime}$. From $\boldsymbol{d} \cdot \boldsymbol{d}^{\prime}=0$ we have $\boldsymbol{d}^{\prime}=\left(\boldsymbol{d}_{\alpha} \cdot \boldsymbol{d}^{\prime}\right) \boldsymbol{d}_{\alpha}$ and $\boldsymbol{d} \times \boldsymbol{d}^{\prime}=\left(\boldsymbol{d}_{\alpha} \cdot \boldsymbol{d}^{\prime}\right) \boldsymbol{d} \times \boldsymbol{d}_{\alpha}=\left(e_{\beta 3 \alpha} \boldsymbol{d}_{\alpha} \cdot \boldsymbol{d}^{\prime}\right) \boldsymbol{d}_{\beta} ;$ thus, $\kappa_{\beta} \boldsymbol{d}_{\beta}=$ $\boldsymbol{d} \times \boldsymbol{d}^{\prime}$ and (21) becomes

$$
\boldsymbol{\mu}=T \tau \boldsymbol{d}+F \boldsymbol{d} \times \boldsymbol{d}^{\prime} .
$$

From (1) we have that $(29)_{2}$ is equivalent to the system

$$
\boldsymbol{d} \cdot\left(\boldsymbol{\mu}^{\prime}+\pi\right)=0 \quad \text { and } \quad \boldsymbol{d} \times\left(\boldsymbol{\mu}^{\prime}+\boldsymbol{\pi}\right)=\lambda \boldsymbol{d} \times(\boldsymbol{f} \times \boldsymbol{d}) .
$$

In the second of these we combine the identity $d \times(f \times d)=f-(d \cdot f) d$ with (27) to obtain

$$
\boldsymbol{f}=W_{\lambda} \boldsymbol{d}+\lambda^{-1} \boldsymbol{d} \times\left(\boldsymbol{\mu}^{\prime}+\boldsymbol{\pi}\right),
$$

which may be combined with $(29)_{1}$ and $(34)_{1}$ to provide an alternative set of equilibrium equations. The latter form of Kirchhoff's theory furnishes a more natural analog to the system derived for a lattice of rods in Sections 3 and 4.

2.4. Three-dimensional lattice. We suppose the three-dimensional continuum to be composed of a continuous distribution of orthogonal rods of the kind discussed in the foregoing. Every point of the continuum is regarded as a point of intersection of three fibers. These are assumed to be aligned, prior to deformation, with the uniform, right-handed orthonormal triad $\{\boldsymbol{L}, \boldsymbol{M}, \boldsymbol{N}\}$. The lattice of fibers is assumed to be rigid in the sense that it remains orthogonal, and similarly oriented, in the course of the deformation. That is, the set $\{\boldsymbol{L}, \boldsymbol{M}, \boldsymbol{N}\}$ of material vectors is stretched and rotated to the (generally nonuniform) set $\left\{\lambda_{l} \boldsymbol{l}, \lambda_{m} \boldsymbol{m}, \lambda_{n} \boldsymbol{n}\right\}$, where $\{\boldsymbol{l}, \boldsymbol{m}, \boldsymbol{n}\}$ is a right-handed orthonormal triad and $\left\{\lambda_{l}, \lambda_{m}, \lambda_{n}\right\}$ are the fiber stretches.

Accordingly, the orientation of the deformed lattice is specified by the rotation field

$$
\boldsymbol{R}=\boldsymbol{l} \otimes \boldsymbol{L}+\boldsymbol{m} \otimes \boldsymbol{M}+\boldsymbol{n} \otimes \boldsymbol{N} .
$$

This furnishes the curvature-twist vectors $\boldsymbol{\kappa}_{l}, \boldsymbol{\kappa}_{m}, \boldsymbol{\kappa}_{n}$ of the constituent fibers in accordance with (9); thus, for example, the curvature-twist vector of a fiber initially aligned with $\boldsymbol{L}$ is $\boldsymbol{\kappa}_{l}=\kappa_{(l) i} \boldsymbol{L}_{i}$, where $\left\{\boldsymbol{L}_{i}\right\}=\left\{\boldsymbol{L}_{\alpha}, \boldsymbol{L}\right\}$ with $\left\{\boldsymbol{L}_{\alpha}\right\}=\{\boldsymbol{M}, \boldsymbol{N}\}$, and

$$
\kappa_{(l) i}=\frac{1}{2} e_{i j k} \boldsymbol{l}_{k} \cdot \boldsymbol{l}_{j}^{\prime},
$$

where $\left\{\boldsymbol{l}_{i}\right\}=\left\{\boldsymbol{l}_{\alpha}, \boldsymbol{l}\right\}$ with $\left\{\boldsymbol{l}_{\alpha}\right\}=\{\boldsymbol{m}, \boldsymbol{n}\}$. Here, $\boldsymbol{l}_{j}^{\prime}=\left(\nabla \boldsymbol{l}_{j}\right) \boldsymbol{L}$ is the directional derivative along the $\boldsymbol{L}$-fiber. Because $\boldsymbol{l}_{i}=\boldsymbol{R} \boldsymbol{L}_{i}$ it is evident that $\boldsymbol{\kappa}_{l}$ is determined by the rotation field $\boldsymbol{R}$ and its gradient. 
In the same way, $\boldsymbol{\kappa}_{m}=\kappa_{(m) i} \boldsymbol{M}_{i}$, where $\left\{\boldsymbol{M}_{i}\right\}=\left\{\boldsymbol{M}_{\alpha}, \boldsymbol{M}\right\}$ with $\left\{\boldsymbol{M}_{\alpha}\right\}=\{\boldsymbol{N}, \boldsymbol{L}\}$, and

$$
\kappa_{(m) i}=\frac{1}{2} e_{i j k} \boldsymbol{m}_{k} \cdot \boldsymbol{m}_{j}^{\prime},
$$

where $\left\{\boldsymbol{m}_{i}\right\}=\left\{\boldsymbol{R} \boldsymbol{M}_{i}\right\}=\left\{\boldsymbol{m}_{\alpha}, \boldsymbol{m}\right\}$, with $\left\{\boldsymbol{m}_{\alpha}\right\}=\{\boldsymbol{n}, \boldsymbol{l}\}$, and where $\boldsymbol{m}_{j}^{\prime}=\left(\nabla \boldsymbol{m}_{j}\right) \boldsymbol{M}$ is now the directional derivative along the $\boldsymbol{M}$-fiber. Finally, $\kappa_{n}=\kappa_{(n) i} \boldsymbol{N}_{i}$, where $\left\{\boldsymbol{N}_{i}\right\}=\left\{\boldsymbol{N}_{\alpha}, \boldsymbol{N}\right\}$ with $\left\{\boldsymbol{N}_{\alpha}\right\}=\{\boldsymbol{L}, \boldsymbol{M}\}$, and

$$
\kappa_{(n) i}=\frac{1}{2} e_{i j k} \boldsymbol{n}_{k} \cdot \boldsymbol{n}_{j}^{\prime},
$$

where $\left\{\boldsymbol{n}_{i}\right\}=\left\{\boldsymbol{R} \boldsymbol{N}_{i}\right\}=\left\{\boldsymbol{n}_{\alpha}, \boldsymbol{n}\right\}$, with $\left\{\boldsymbol{n}_{\alpha}\right\}=\{\boldsymbol{l}, \boldsymbol{m}\}$ and $\boldsymbol{n}_{j}^{\prime}=\left(\nabla \boldsymbol{n}_{j}\right) \boldsymbol{N}$. Thus, all three curvature-twist vectors are determined by the single rotation field $\boldsymbol{R}$ and its gradient.

Because the fibers are convected as material curves, we have $\lambda_{l} \boldsymbol{l}=\boldsymbol{F} \boldsymbol{L}$, etc., where $\boldsymbol{F}=\nabla \chi$ is the gradient of the deformation $\chi(\boldsymbol{X})$ of the continuum. Here $\boldsymbol{X}$ is the position of a material point in a reference configuration, $\kappa$ say. The orthonormality of $\{\boldsymbol{L}, \boldsymbol{M}, \boldsymbol{N}\}$ then furnishes

$$
\boldsymbol{F}=\lambda_{l} \boldsymbol{l} \otimes \boldsymbol{L}+\lambda_{m} \boldsymbol{m} \otimes \boldsymbol{M}+\lambda_{n} \boldsymbol{n} \otimes \boldsymbol{N},
$$

where $\lambda_{l}=|\boldsymbol{F} \boldsymbol{L}|$, etc. Evidently,

$$
\boldsymbol{F}=\boldsymbol{R} \boldsymbol{U}
$$

where

$$
\boldsymbol{U}=\lambda_{l} \boldsymbol{L} \otimes \boldsymbol{L}+\lambda_{m} \boldsymbol{M} \otimes \boldsymbol{M}+\lambda_{n} \boldsymbol{N} \otimes \boldsymbol{N}
$$

is positive definite and symmetric. The Cosserat rotation (36) thus coincides with the rotation in the polar factorization of the deformation gradient in which $\boldsymbol{U}$ is the associated right-stretch tensor. Because $\boldsymbol{R}$ is uniquely determined by $\boldsymbol{F}$ in this case, the curvature-twist vectors of the fibers are ultimately determined by the first and second deformation gradients $\nabla \chi$ and $\nabla \nabla \chi$. It is this fact which yields the reduction, detailed in Section 4 , of the Cosserat continuum model outlined in Section 3 to a special second-gradient model of elasticity. Moreover, the present model furnishes a rare example of a material for which the principal axes of strain are fixed in the body.

It may be observed that the kinematical structure of the present three-dimensional framework is not entirely analogous to that of rod theory. This is due to the partial coupling between deformation and rotation implied by (1), whereas in the present three-dimensional theory the relevant rotation field is controlled entirely by the continuum deformation.

We note that if the fibers are inextensible, i.e., if $\lambda_{l}=\lambda_{m}=\lambda_{n}=1$, then $\boldsymbol{U}=\boldsymbol{I}$, $\nabla \chi=\boldsymbol{R}$, and the deformation is necessarily rigid [Gurtin 1981]. Here $\boldsymbol{I}$ is the three-dimensional identity. Thus, nontrivial deformations necessarily entail fiber 
extension or contraction. Accordingly, we expect a lattice material of the kind envisaged to be extremely stiff — the raison d'être of mechanical metamaterials if the extensional stiffnesses of the fibers are large. In contrast, Kirchhoff's theory accommodates nonrigid inextensional deformations.

\section{Cosserat elasticity}

3.1. Kinematics. In the foregoing we have argued that the lattice material may be regarded as a Cosserat continuum endowed with a rotation field $\boldsymbol{R}(\boldsymbol{X})$. This rotation is determined by the deformation $\chi(\boldsymbol{X})$. However, in the present section we regard these fields as being independent in the spirit of the conventional Cosserat theory. The rotation and deformation are ultimately reconnected in Section 4. Existence theory for Cosserat elasticity is discussed in [Neff 2006].

Thus, we introduce a referential energy density $U(\boldsymbol{F}, \boldsymbol{R}, \nabla \boldsymbol{R} ; \boldsymbol{X})$, where $\boldsymbol{F}$ is the usual deformation gradient and $\nabla \boldsymbol{R}$ is the rotation gradient; i.e.,

$$
\boldsymbol{F}=F_{i A} \boldsymbol{e}_{i} \otimes \boldsymbol{E}_{A}, \quad \boldsymbol{R}=R_{i A} \boldsymbol{e}_{i} \otimes \boldsymbol{E}_{A}, \quad \text { and } \quad \nabla \boldsymbol{R}=R_{i A, B} \boldsymbol{e}_{i} \otimes \boldsymbol{E}_{A} \otimes \boldsymbol{E}_{B}
$$

with

$$
F_{i A}=\chi_{i, A}
$$

where $(\cdot),{ }_{A}=\partial(\cdot) / \partial X_{A}$ and we use a Cartesian index notation that emphasizes the two-point character of the deformation gradient and rotation fields. Here $\left\{\boldsymbol{e}_{i}\right\}$ and $\left\{\boldsymbol{E}_{A}\right\}$ are fixed orthonormal bases associated with the Cartesian coordinates $x_{i}$ and $X_{A}$, where $x_{i}=\chi_{i}\left(X_{A}\right)$.

3.2. Strain-energy function. We suppose the strain energy to be Galilean invariant and thus require that

$$
U(\boldsymbol{F}, \boldsymbol{R}, \nabla \boldsymbol{R} ; \boldsymbol{X})=U(\boldsymbol{Q F}, \boldsymbol{Q R}, \boldsymbol{Q} \nabla \boldsymbol{R} ; \boldsymbol{X}),
$$

where $\boldsymbol{Q}$ is an arbitrary spatially uniform rotation and $(\boldsymbol{Q} \nabla \boldsymbol{R})_{i A B}=\left(Q_{i j} R_{j A}\right),_{B}=$ $Q_{i j} R_{j A, B}$. The restriction

$$
U(\boldsymbol{F}, \boldsymbol{R}, \nabla \boldsymbol{R} ; \boldsymbol{X})=W(\boldsymbol{E}, \boldsymbol{\Gamma} ; \boldsymbol{X}),
$$

where [Pietraszkiewicz and Eremeyev 2009; Eremeyev and Pietraszkiewicz 2012; Steigmann 2012; 2015]

$$
\begin{aligned}
& \boldsymbol{E}=\boldsymbol{R}^{t} \boldsymbol{F}=E_{A B} \boldsymbol{E}_{A} \otimes \boldsymbol{E}_{B}, \quad E_{A B}=R_{i A} F_{i B}, \\
& \boldsymbol{\Gamma}=\Gamma_{D C} \boldsymbol{E}_{D} \otimes \boldsymbol{E}_{C}, \quad \Gamma_{D C}=\frac{1}{2} e_{B A D} R_{i A} R_{i B, C},
\end{aligned}
$$

with $W$ a suitable function and $e_{A B C}$ the permutation symbol, furnishes the necessary and sufficient condition for Galilean invariance. Sufficiency is obvious; necessity follows by choosing $\boldsymbol{Q}=\boldsymbol{R}_{\mid X}^{t}$, where $X$ is the material point in question, 
and making use of the fact that, for each fixed $C \in\{1,2,3\}$, the matrix $R_{i A} R_{i B, C}$ is skew. This follows by differentiating $R_{i A} R_{i B}=\delta_{A B}$ (the Kronecker delta). The associated axial vectors $\boldsymbol{\gamma}_{C}$ have components

$$
\gamma_{D(C)}=\frac{1}{2} e_{B A D} R_{i A} R_{i B, C},
$$

yielding [Pietraszkiewicz and Eremeyev 2009]

$$
\Gamma=\gamma_{C} \otimes E_{C},
$$

and so $\boldsymbol{\Gamma}$ - the wryness tensor - stands in one-to-one relation to the Cosserat strain measure $\boldsymbol{R}^{t} \nabla \boldsymbol{R}$.

3.3. Virtual power and equilibrium. We define equilibria to be states that satisfy the virtual-power statement

$$
\dot{E}_{\pi}=P_{\pi},
$$

where $\pi$ is an arbitrary subvolume of $\kappa, P_{\pi}$ is the virtual power of the loads acting thereon,

$$
E_{\pi}=\int_{\pi} U d v
$$

is the strain energy contained in $\pi$, and superposed dots identify variational derivatives, as in Section 2. Thus,

$$
\dot{U}=\dot{W}=\sigma \cdot \dot{\boldsymbol{E}}+\boldsymbol{\mu} \cdot \dot{\boldsymbol{\Gamma}},
$$

where

$$
\sigma=W_{\boldsymbol{E}} \quad \text { and } \quad \boldsymbol{\mu}=W_{\Gamma}
$$

are evaluated at equilibrium and the variational derivatives are evaluated at an equilibrium state. The dots interposed between the terms in (53) represent the standard Euclidean inner product on the linear space of tensors. The explicit form of $P_{\pi}$ is deduced below.

From (47) we have

$$
\dot{\boldsymbol{E}}=\boldsymbol{R}^{t}(\nabla \boldsymbol{u}-\boldsymbol{\Omega} \boldsymbol{F}), \quad \text { where } \boldsymbol{u}=\dot{\chi} \text { and } \boldsymbol{\Omega}=\dot{\boldsymbol{R}} \boldsymbol{R}^{t} .
$$

Then,

$$
\boldsymbol{\sigma} \cdot \dot{\boldsymbol{E}}=\boldsymbol{R} \boldsymbol{\sigma} \cdot \nabla \boldsymbol{u}-\boldsymbol{\Omega} \cdot \operatorname{Skw}\left(\boldsymbol{R} \boldsymbol{\sigma} \boldsymbol{F}^{t}\right) .
$$

Here, of course, $\boldsymbol{\Omega}$ is skew. Let $\boldsymbol{\omega}=\operatorname{ax} \boldsymbol{\Omega}$ be its axial vector, defined, for arbitrary $\boldsymbol{v}$, by $\boldsymbol{\omega} \times \boldsymbol{v}=\boldsymbol{\Omega} \boldsymbol{v}$. If $\boldsymbol{\alpha}$ is a skew tensor and $\boldsymbol{a}=\operatorname{ax\boldsymbol {\alpha }}$, then, as is well known, $\boldsymbol{\Omega} \cdot \boldsymbol{\alpha}=2 \boldsymbol{\omega} \cdot \boldsymbol{a}$. Further, $\boldsymbol{R} \boldsymbol{\sigma} \boldsymbol{F}^{t}=\boldsymbol{R} \boldsymbol{\sigma} \boldsymbol{E}^{t} \boldsymbol{R}^{t}$ and $\operatorname{Skw}\left(\boldsymbol{R} \boldsymbol{\sigma} \boldsymbol{E}^{t} \boldsymbol{R}^{t}\right)=\boldsymbol{R}\left(\operatorname{Skw} \boldsymbol{\sigma} \boldsymbol{E}^{t}\right) \boldsymbol{R}^{t}$ so that, finally,

$$
\boldsymbol{\sigma} \cdot \dot{\boldsymbol{E}}=\boldsymbol{R} \boldsymbol{\sigma} \cdot \nabla \boldsymbol{u}-2 a x\left[\boldsymbol{R}\left(\mathrm{Skw} \boldsymbol{\sigma} \boldsymbol{E}^{t}\right) \boldsymbol{R}^{t}\right] \cdot \boldsymbol{\omega} .
$$


To reduce $\boldsymbol{\mu} \cdot \dot{\boldsymbol{\Gamma}}$ we make use of the formula [Steigmann 2012]

$$
\boldsymbol{\mu} \cdot \dot{\boldsymbol{\Gamma}}=\bar{\omega}_{E}\left(\mu_{E C, C}+e_{E D B} \mu_{B C} \Gamma_{D C}\right)-\left(\bar{\omega}_{E} \mu_{E C}\right)_{, C},
$$

where $\overline{\boldsymbol{\omega}}=-\boldsymbol{R}^{t} \boldsymbol{\omega}$ is the axial vector of $\dot{\boldsymbol{R}}^{t} \boldsymbol{R}$. This may be recast in the form

$$
\boldsymbol{\mu} \cdot \dot{\boldsymbol{\Gamma}}=\operatorname{Div}\left(\boldsymbol{\mu}^{t} \boldsymbol{R}^{t} \boldsymbol{\omega}\right)-\boldsymbol{\omega} \cdot \operatorname{Div}(\boldsymbol{R} \boldsymbol{\mu})+\omega_{i} \mu_{E C}\left(R_{i E, C}-e_{B D E} R_{i B} \Gamma_{D C}\right) .
$$

The inverse of $(48)_{2}$ is

$$
e_{B D E} \Gamma_{D C}=R_{j B} R_{j E, C} ; \quad \text { thus, } \quad e_{B D E} R_{i B} \Gamma_{D C}=\delta_{i j} R_{j E, C},
$$

implying that the last term of (59) vanishes and hence that

$$
\boldsymbol{\mu} \cdot \dot{\boldsymbol{\Gamma}}=\boldsymbol{R} \boldsymbol{\mu} \cdot \nabla \boldsymbol{\omega} .
$$

Substitution of (57) and (61) into (51) furnishes

$P_{\pi}=\int_{\partial \pi} \boldsymbol{R} \boldsymbol{\mu \nu} \cdot \boldsymbol{\omega} d a-\int_{\pi}\left\{\boldsymbol{R} \boldsymbol{\sigma} \cdot \nabla \boldsymbol{u}+\boldsymbol{\omega} \cdot\left[\operatorname{Div}(\boldsymbol{R} \boldsymbol{\mu})+2 \operatorname{ax}\left(\boldsymbol{R}\left(\operatorname{Skw} \boldsymbol{\sigma} \boldsymbol{E}^{t}\right) \boldsymbol{R}^{t}\right)\right]\right\} d v$,

where $\boldsymbol{v}$ is the exterior unit normal to the piecewise smooth surface $2 \pi$. The virtual power is thus of the form

$$
P_{\pi}=\int_{\partial \pi}(\boldsymbol{t} \cdot \boldsymbol{u}+\boldsymbol{c} \cdot \boldsymbol{\omega}) d a+\int_{\pi}(\boldsymbol{g} \cdot \boldsymbol{u}+\boldsymbol{\pi} \cdot \boldsymbol{\omega}) d v,
$$

where $\boldsymbol{t}$ and $\boldsymbol{c}$ are densities of force and couple acting on $\partial \pi$, and $\boldsymbol{g}$ and $\boldsymbol{\pi}$ are densities of force and couple acting in $\pi$.

If $\boldsymbol{u}$ and $\boldsymbol{\omega}$ are independent and if there are no kinematical constraints, then by the fundamental lemma,

$$
\boldsymbol{t}=\boldsymbol{R} \boldsymbol{\sigma} \boldsymbol{v} \quad \text { and } \quad \boldsymbol{c}=\boldsymbol{R} \boldsymbol{\mu \nu} \text { on } \partial \pi,
$$

and

$$
\boldsymbol{g}=-\operatorname{Div}(\boldsymbol{R} \boldsymbol{\sigma}) \quad \text { and } \quad \boldsymbol{\pi}=-\operatorname{Div}(\boldsymbol{R} \boldsymbol{\mu})-2 \operatorname{ax}\left[\boldsymbol{R}\left(\operatorname{Skw} \boldsymbol{\sigma} \boldsymbol{E}^{t}\right) \boldsymbol{R}^{t}\right] \quad \text { in } \pi .
$$

These are the equilibrium conditions for a standard Cosserat continuum in which the deformation $\chi$ and rotation $\boldsymbol{R}$ are independent kinematical fields. The use of the axial vector $\omega$ in their derivation yields a simpler set of equations than that derived in [Reissner 1975; 1987; Steigmann 2012; 2015] on the basis of the axial vector $\overline{\boldsymbol{\omega}}$.

3.4. Specialization to an orthogonal lattice. The curvature-twist vector $\kappa_{l}$ of a fiber initially aligned with $\boldsymbol{L}$ may be described in the present framework by using (36) and (37) to write

$$
\kappa_{(l) i}=\frac{1}{2} e_{i j k} \boldsymbol{L}_{k} \cdot \boldsymbol{R}^{t} \boldsymbol{R}^{\prime} \boldsymbol{L}_{j}
$$


where $(\cdot)^{\prime}$ is the directional derivative along $\boldsymbol{L}$ and we have assumed that the fibers are initially straight and untwisted, i.e., that $\boldsymbol{L}_{j}^{\prime}=\mathbf{0}$. Here we use $R_{i A}^{\prime}=R_{i A, B} L_{B}$ to derive (see $(60)_{1}$ )

$$
\boldsymbol{R}^{t} \boldsymbol{R}^{\prime}=R_{i C} R_{i A, B} L_{B} \boldsymbol{E}_{C} \otimes \boldsymbol{E}_{A}=e_{A C D} \Gamma_{D B} L_{B} \boldsymbol{E}_{C} \otimes \boldsymbol{E}_{A},
$$

which implies that $\kappa_{l}=\kappa_{(l) i} \boldsymbol{L}_{i}$ is determined by $\boldsymbol{\Gamma}$ via $\boldsymbol{\Gamma} \boldsymbol{L}$. In the same way, $\boldsymbol{\kappa}_{m}$ and $\boldsymbol{\kappa}_{n}$ are determined by $\boldsymbol{\Gamma}$ via $\boldsymbol{\Gamma} \boldsymbol{M}$ and $\boldsymbol{\Gamma} \boldsymbol{N}$, respectively.

Moreover, from (41) and (47) we find that $\boldsymbol{E}=\boldsymbol{U}$ in the case of an orthogonal lattice. The fiber stretches are thus given by

$$
\lambda_{l}=\boldsymbol{L} \otimes \boldsymbol{L} \cdot \boldsymbol{E}, \quad \lambda_{m}=\boldsymbol{M} \otimes \boldsymbol{M} \cdot \boldsymbol{E}, \quad \lambda_{m}=\boldsymbol{N} \otimes \boldsymbol{N} \cdot \boldsymbol{E} .
$$

In a discrete lattice consisting of spatial rods interacting at interior nodes as in [Steigmann 1996], the strain energy is the sum of the strain energies of the individual rods. This motivates the assumption of an additive decomposition of the strain energy in the continuum lattice model; i.e.,

$$
W(\boldsymbol{E}, \boldsymbol{\Gamma})=W_{l}\left(\lambda_{l}, \boldsymbol{\kappa}_{l}\right)+W_{m}\left(\lambda_{m}, \boldsymbol{\kappa}_{m}\right)+W_{n}\left(\lambda_{n}, \boldsymbol{\kappa}_{n}\right),
$$

in which $W_{l, m, n}$ are the strain energies, per unit initial volume, of the three fiber families.

Using (54) $)_{1}$ and (68) we then derive

$$
\boldsymbol{\sigma}=\boldsymbol{\sigma}_{l} \otimes \boldsymbol{L}+\boldsymbol{\sigma}_{m} \otimes \boldsymbol{M}+\boldsymbol{\sigma}_{n} \otimes \boldsymbol{N},
$$

with

yielding

$$
\boldsymbol{\sigma}_{l}=\frac{\partial W_{l}}{\partial \lambda_{l}} \boldsymbol{L}, \quad \boldsymbol{\sigma}_{m}=\frac{\partial W_{m}}{\partial \lambda_{m}} \boldsymbol{M}, \quad \boldsymbol{\sigma}_{n}=\frac{\partial W_{n}}{\partial \lambda_{n}} \boldsymbol{N},
$$

$$
\boldsymbol{\sigma} \boldsymbol{E}^{t}=\lambda_{l} \frac{\partial W_{l}}{\partial \lambda_{l}} \boldsymbol{L} \otimes \boldsymbol{L}+\lambda_{m} \frac{\partial W_{m}}{\partial \lambda_{m}} \boldsymbol{M} \otimes \boldsymbol{M}+\lambda_{n} \frac{\partial W_{n}}{\partial \lambda_{n}} \boldsymbol{N} \otimes \boldsymbol{N}
$$

We thus have $\operatorname{Skw}\left(\boldsymbol{\sigma} \boldsymbol{E}^{t}\right)=\mathbf{0}$ and conclude that the associated interaction term vanishes in $(65)_{2}$. In general the latter may be interpreted as a distributed moment transmitted to the fibers by a matrix material in which the fibers are embedded [Steigmann 2012]. However, the relatively simple model discussed here does not take account of an underlying matrix.

To derive the relevant expression for the couple stress $\boldsymbol{\mu}$ we use (18), for the fiber family initially aligned with $\boldsymbol{L}$, in the form

$$
\dot{\kappa}_{(l) i}=\boldsymbol{l}_{i} \cdot(\nabla \boldsymbol{\omega}) \boldsymbol{L}=\boldsymbol{R} \boldsymbol{L}_{i} \otimes \boldsymbol{L} \cdot \nabla \boldsymbol{\omega} .
$$

Thus,

$$
\frac{\partial W_{l}}{\partial \kappa_{(l) i}} \dot{\kappa}_{(l) i}=\boldsymbol{R} \boldsymbol{\mu}_{l} \otimes \boldsymbol{L} \cdot \nabla \boldsymbol{\omega}, \quad \text { with } \boldsymbol{\mu}_{l}=\frac{\partial W_{l}}{\partial \kappa_{(l) i}} \boldsymbol{L}_{i} .
$$


Proceeding from $(54)_{2}$ and (61) in the same way, we find that

$$
W_{\boldsymbol{\Gamma}} \cdot \dot{\boldsymbol{\Gamma}}=\boldsymbol{R}\left(\boldsymbol{\mu}_{l} \otimes \boldsymbol{L}+\boldsymbol{\mu}_{m} \otimes \boldsymbol{M}+\boldsymbol{\mu}_{n} \otimes \boldsymbol{N}\right) \cdot \nabla \boldsymbol{\omega},
$$

with

$$
\boldsymbol{\mu}_{m}=\frac{\partial W_{m}}{\partial \kappa_{(m) i}} \boldsymbol{M}_{i} \quad \text { and } \quad \boldsymbol{\mu}_{n}=\frac{\partial W_{n}}{\partial \kappa_{(n) i}} \boldsymbol{N}_{i}
$$

and comparison with (61) furnishes

$$
\boldsymbol{\mu}=\boldsymbol{\mu}_{l} \otimes L+\boldsymbol{\mu}_{m} \otimes \boldsymbol{M}+\boldsymbol{\mu}_{n} \otimes N .
$$

In the virtual-power statement (51) we then have

$$
\dot{E}_{\pi}=\int_{\partial \pi} \boldsymbol{R} \boldsymbol{\mu} \boldsymbol{v} \cdot \boldsymbol{\omega} d a+\int_{\pi}[\boldsymbol{R} \boldsymbol{\sigma} \cdot \nabla \boldsymbol{u}-\boldsymbol{\omega} \cdot \operatorname{Div}(\boldsymbol{R} \boldsymbol{\mu})] d v,
$$

where

$$
\operatorname{Div}(\boldsymbol{R} \boldsymbol{\sigma})=\left[\nabla\left(\boldsymbol{R} \boldsymbol{\sigma}_{l}\right)\right] \boldsymbol{L}+\left[\nabla\left(\boldsymbol{R} \boldsymbol{\sigma}_{m}\right)\right] \boldsymbol{M}+\left[\nabla\left(\boldsymbol{R} \boldsymbol{\sigma}_{n}\right)\right] \boldsymbol{N}
$$

and

$$
\operatorname{Div}(\boldsymbol{R} \boldsymbol{\mu})=\left[\nabla\left(\boldsymbol{R} \boldsymbol{\mu}_{l}\right)\right] \boldsymbol{L}+\left[\nabla\left(\boldsymbol{R} \boldsymbol{\mu}_{m}\right)\right] \boldsymbol{M}+\left[\nabla\left(\boldsymbol{R} \boldsymbol{\mu}_{n}\right)\right] \boldsymbol{N} .
$$

The equilibrium equations are not obtained by substituting into (64) and (65), however, because the virtual translational velocity $\boldsymbol{u}$ and rotational velocity $\boldsymbol{\omega}$ are not independent.

Suppose, for example, that all three fiber families have identical uniform properties, each with a strain-energy function of the form (11). Then (see $(71)_{1}$ and $\left.(74)_{2}\right)$,

$$
\boldsymbol{R} \boldsymbol{\sigma}_{l}=E\left(\lambda_{l}-1\right) \boldsymbol{l} \quad \text { and } \quad \boldsymbol{R} \boldsymbol{\mu}_{l}=T \tau_{l} \boldsymbol{l}+F \boldsymbol{l} \times(\nabla \boldsymbol{l}) \boldsymbol{L}, \quad \text { etc. }
$$

where $\tau_{l}=\kappa_{(l) 3}$, etc., in which $E, T$, and $F$ respectively are the constant extensional, torsional, and flexural stiffnesses of the fibers. Again we note that the torsional and flexural stiffnesses could conceivably depend on fiber stretch. However, for small extensional strains they are approximated at leading order by constants.

\section{Reduction to second-gradient elasticity}

\subsection{Reducing a linear form in the rotational virtual velocity to a linear form in} the gradient of the translational virtual velocity. To effect the reduction of the Cosserat model to a second-gradient elasticity model [Toupin 1964; Mindlin and Tiersten 1962; Koiter 1964], we proceed from (36) to write the virtual spin tensor $\boldsymbol{\Omega}=\dot{\boldsymbol{R}} \boldsymbol{R}^{t}$ in the form

$$
\boldsymbol{\Omega}=\dot{\boldsymbol{l}} \otimes \boldsymbol{l}+\dot{\boldsymbol{m}} \otimes \boldsymbol{m}+\dot{\boldsymbol{n}} \otimes \boldsymbol{n},
$$

where, from (40),

$$
\lambda_{l} \boldsymbol{l}=(\nabla \boldsymbol{u}) \boldsymbol{L}-\dot{\lambda}_{l} \boldsymbol{l}, \quad \text { etc. }
$$


Its axial vector $\omega$ is such that

$$
\omega \times v=(v \cdot l) \dot{l}+(v \cdot m) \dot{m}+(v \cdot n) \dot{n}
$$

for every vector $\boldsymbol{v}$. Using the well known identity

$$
\boldsymbol{a} \times(\boldsymbol{b} \times \boldsymbol{c})=(\boldsymbol{a} \cdot \boldsymbol{c}) \boldsymbol{b}-(\boldsymbol{a} \cdot \boldsymbol{b}) \boldsymbol{c},
$$

we derive

$$
(\boldsymbol{v} \cdot \boldsymbol{l}) \dot{l}=(\boldsymbol{l} \times \boldsymbol{i}) \times \boldsymbol{v}+(\boldsymbol{v} \cdot \dot{l}) \boldsymbol{l}, \quad \text { etc., }
$$

yielding

$$
\omega \times \boldsymbol{v}=(\boldsymbol{l} \times \dot{\boldsymbol{l}}+\boldsymbol{m} \times \dot{\boldsymbol{m}}+\boldsymbol{n} \times \dot{\boldsymbol{n}}) \times \boldsymbol{v}+\boldsymbol{\Omega}^{t} \boldsymbol{v},
$$

and thus conclude that

$$
\omega=\frac{1}{2}(\boldsymbol{l} \times \dot{\boldsymbol{l}}+\boldsymbol{m} \times \dot{\boldsymbol{m}}+\boldsymbol{n} \times \dot{\boldsymbol{n}}) .
$$

In the virtual-power statement (78) we have two linear expressions of the form $\boldsymbol{a} \cdot \boldsymbol{\omega}$. We write these as

$$
\boldsymbol{a} \cdot \omega=\frac{1}{2}(\boldsymbol{a} \times \boldsymbol{l} \cdot \dot{\boldsymbol{l}}+\boldsymbol{a} \times \boldsymbol{m} \cdot \dot{\boldsymbol{m}}+\boldsymbol{a} \times \boldsymbol{n} \cdot \dot{\boldsymbol{n}}),
$$

where

$$
\boldsymbol{a} \times \boldsymbol{l} \cdot \boldsymbol{l}=\lambda_{l}^{-1} \boldsymbol{a} \times \boldsymbol{l} \cdot(\nabla \boldsymbol{u}) \boldsymbol{L}=\lambda_{l}^{-1}(\boldsymbol{a} \times \boldsymbol{l}) \otimes \boldsymbol{L} \cdot \nabla \boldsymbol{u}, \quad \text { etc. }
$$

Altogether,

$$
\boldsymbol{a} \cdot \boldsymbol{\omega}=\frac{1}{2}\left[\lambda_{l}^{-1}(\boldsymbol{a} \times \boldsymbol{l}) \otimes \boldsymbol{L}+\lambda_{m}^{-1}(\boldsymbol{a} \times \boldsymbol{m}) \otimes \boldsymbol{M}+\lambda_{n}^{-1}(\boldsymbol{a} \times \boldsymbol{n}) \otimes N\right] \cdot \nabla \boldsymbol{u} .
$$

Every linear scalar-valued function of $\omega$ may thus be expressed as a linear function of $\nabla \boldsymbol{u}$. We thereby reduce (78) to

$$
\dot{E}_{\pi}=\int_{\pi} \boldsymbol{P} \cdot \nabla \boldsymbol{u} d v+\int_{\partial \pi} \boldsymbol{Q} \cdot \nabla \boldsymbol{u} d a,
$$

where

$$
\begin{array}{r}
\boldsymbol{P}=\boldsymbol{R} \boldsymbol{\sigma}-\frac{1}{2}\left[\lambda_{l}^{-1}(\boldsymbol{a} \times \boldsymbol{l}) \otimes \boldsymbol{L}+\lambda_{m}^{-1}(\boldsymbol{a} \times \boldsymbol{m}) \otimes \boldsymbol{M}+\lambda_{n}^{-1}(\boldsymbol{a} \times \boldsymbol{n}) \otimes \boldsymbol{N}\right], \\
\text { with } \boldsymbol{a}=\operatorname{Div}(\boldsymbol{R} \boldsymbol{\mu}),
\end{array}
$$

and

$$
\boldsymbol{Q}=\frac{1}{2}\left[\lambda_{l}^{-1}(\boldsymbol{b} \times \boldsymbol{l}) \otimes \boldsymbol{L}+\lambda_{m}^{-1}(\boldsymbol{b} \times \boldsymbol{m}) \otimes \boldsymbol{M}+\lambda_{n}^{-1}(\boldsymbol{b} \times \boldsymbol{n}) \otimes \boldsymbol{N}\right], \quad \text { with } \boldsymbol{b}=\boldsymbol{R} \boldsymbol{\mu \nu} . \text { (94) }
$$


4.2. Constraints on the virtual velocity gradient and the extended variational problem. The translational virtual velocity gradient is subject to further constraints associated with the rigid connectivity of the lattice. This implies that (see (36) and (40))

$$
\boldsymbol{F} \boldsymbol{L} \cdot \boldsymbol{F} \boldsymbol{M}=0, \quad \boldsymbol{F} \boldsymbol{L} \cdot \boldsymbol{F} \boldsymbol{N}=0, \quad \text { and } \quad \boldsymbol{F} \boldsymbol{M} \cdot \boldsymbol{F} \boldsymbol{N}=0 .
$$

To accommodate these we replace the virtual-power statement (51) by

$$
\dot{E}_{\pi}^{*}=P_{\pi},
$$

where

$$
E_{\pi}^{*}=E_{\pi}+\int_{\pi} \frac{1}{2} \boldsymbol{\Lambda} \cdot \boldsymbol{C} d v+\int_{\partial \pi} \frac{1}{2} \mathbf{\Xi} \cdot \boldsymbol{C} d a,
$$

is the extended energy, $\boldsymbol{C}=\boldsymbol{F}^{t} \boldsymbol{F}$ is the right Cauchy-Green deformation tensor, and

$$
\begin{aligned}
& \boldsymbol{\Lambda}=\Lambda_{L M}(\boldsymbol{L} \otimes \boldsymbol{M}+\boldsymbol{M} \otimes \boldsymbol{L})+\Lambda_{L N}(\boldsymbol{L} \otimes \boldsymbol{N}+\boldsymbol{N} \otimes \boldsymbol{L})+\Lambda_{M N}(\boldsymbol{M} \otimes \boldsymbol{N}+\boldsymbol{N} \otimes \boldsymbol{M}), \\
& \boldsymbol{\Xi}=\mathbf{\Xi}_{L M}(\boldsymbol{L} \otimes \boldsymbol{M}+\boldsymbol{M} \otimes \boldsymbol{L})+\Xi_{L N}(\boldsymbol{L} \otimes \boldsymbol{N}+\boldsymbol{N} \otimes \boldsymbol{L})+\mathbf{\Xi}_{M N}(\boldsymbol{M} \otimes \boldsymbol{N}+\boldsymbol{N} \otimes \boldsymbol{M}),
\end{aligned}
$$

in which $\Lambda_{L M}, \Xi_{L M}$, etc., are Lagrange multipliers. We require multipliers on $\partial \pi$ because the gradient of $\boldsymbol{u}$ thereon, which figures in the virtual-work statement (see (92)), is restricted by (95). Their role in the theory is illustrated in Section 5.

Equation (96) is treated as an unconstrained variational problem in which the additional terms have the variational derivatives

$$
\frac{1}{2} \boldsymbol{\Lambda} \cdot \dot{\boldsymbol{C}}=\boldsymbol{F} \boldsymbol{\Lambda} \cdot \nabla \boldsymbol{u} \quad \text { and } \quad \frac{1}{2} \boldsymbol{\Xi} \cdot \dot{\boldsymbol{C}}=\boldsymbol{F} \boldsymbol{\Xi} \cdot \nabla \boldsymbol{u}
$$

at fixed values of the multipliers, whereas variations with respect to the latter merely return the constraints and, as before, are not made explicit. Finally, in (96) we have

where

$$
\dot{E}_{\pi}=\int_{\pi} \boldsymbol{T} \cdot \nabla \boldsymbol{u} d v+\int_{\partial \pi} \boldsymbol{S} \cdot \nabla \boldsymbol{u} d a
$$

$$
\boldsymbol{T}=\boldsymbol{P}+\boldsymbol{F} \boldsymbol{\Lambda} \quad \text { and } \quad \boldsymbol{S}=\boldsymbol{Q}+\boldsymbol{F} \boldsymbol{\Xi}
$$

and it is understood that these are evaluated with the constraints (95) in force.

It is useful to observe, from (101), (93), and (70), that

$$
\boldsymbol{T}=\boldsymbol{T}_{l} \otimes \boldsymbol{L}+\boldsymbol{T}_{m} \otimes \boldsymbol{M}+\boldsymbol{T}_{n} \otimes \boldsymbol{N},
$$

where

$$
\begin{gathered}
\boldsymbol{T}_{l}=\boldsymbol{R} \boldsymbol{\sigma}_{l}+\lambda_{m} \Lambda_{L M} \boldsymbol{m}+\lambda_{n} \Lambda_{L N} \boldsymbol{n}+\frac{1}{2} \lambda_{l}^{-1} \boldsymbol{l} \times \boldsymbol{a}, \\
\boldsymbol{T}_{m}=\boldsymbol{R} \boldsymbol{\sigma}_{m}+\lambda_{l} \Lambda_{L M} \boldsymbol{l}+\lambda_{n} \Lambda_{M N} \boldsymbol{n}+\frac{1}{2} \lambda_{m}^{-1} \boldsymbol{m} \times \boldsymbol{a}, \\
\boldsymbol{T}_{n}=\boldsymbol{R} \boldsymbol{\sigma}_{n}+\lambda_{l} \Lambda_{L N} \boldsymbol{l}+\lambda_{m} \Lambda_{M N} \boldsymbol{m}+\frac{1}{2} \lambda_{n}^{-1} \boldsymbol{n} \times \boldsymbol{a},
\end{gathered}
$$


and $\boldsymbol{a}$ is given by $(93)_{2}$. Similarly,

$$
S=S_{l} \otimes L+S_{m} \otimes M+S_{n} \otimes N
$$

where

$$
\begin{gathered}
\boldsymbol{S}_{l}=\lambda_{m} \boldsymbol{\Xi}_{L M} \boldsymbol{m}+\lambda_{n} \Xi_{L N} \boldsymbol{n}+\frac{1}{2} \lambda_{l}^{-1} \boldsymbol{b} \times \boldsymbol{l}, \\
\boldsymbol{S}_{m}=\lambda_{l} \boldsymbol{\Xi}_{L M} \boldsymbol{l}+\lambda_{n} \Xi_{M N} \boldsymbol{n}+\frac{1}{2} \lambda_{m}^{-1} \boldsymbol{b} \times \boldsymbol{m}, \\
\boldsymbol{S}_{n}=\lambda_{l} \boldsymbol{\Xi}_{L N} \boldsymbol{l}+\lambda_{m} \Xi_{M N} \boldsymbol{m}+\frac{1}{2} \lambda_{n}^{-1} \boldsymbol{b} \times \boldsymbol{n},
\end{gathered}
$$

and $\boldsymbol{b}$ is given by $(94)_{2}$.

4.3. Equilibrium conditions. Equation (100) involves the restriction to the boundary of the gradient of the translational virtual velocity. To treat this we introduce the surface parametrization $\boldsymbol{X}\left(\theta^{\alpha}\right)$ of $\partial \pi$, where $\theta^{\alpha}(\alpha=1,2)$ is a system of convected curvilinear coordinates. This induces the tangent basis $\boldsymbol{A}_{\alpha}=\boldsymbol{X}_{, \alpha}$ and dual tangent basis $\boldsymbol{A}^{\alpha}$, which we use to decompose $\nabla \boldsymbol{u}_{\mid \partial \pi}$ as

$$
\nabla \boldsymbol{u}=\boldsymbol{u}_{, \alpha} \otimes A^{\alpha}+\boldsymbol{u}_{v} \otimes \boldsymbol{v},
$$

where $\boldsymbol{u}_{, \alpha}=\partial \boldsymbol{u}\left(\boldsymbol{X}\left(\theta^{\beta}\right)\right) / \partial \theta^{\alpha}=(\nabla \boldsymbol{u}) \boldsymbol{A}_{\alpha}$ are the tangential derivatives of $\boldsymbol{u}$ and $\boldsymbol{u}_{v}=(\nabla \boldsymbol{u}) \boldsymbol{v}$ is the normal derivative. Thus,

$$
\boldsymbol{S} \cdot \nabla \boldsymbol{u}=\boldsymbol{S} \boldsymbol{v} \cdot \boldsymbol{u}_{v}+\boldsymbol{S}^{\alpha} \cdot \boldsymbol{u}_{, \alpha},
$$

where $\boldsymbol{S}^{\alpha}=\boldsymbol{S} \boldsymbol{A}^{\alpha}$.

Because $\partial \pi$ is piecewise smooth it is the union of a finite number of smooth subsurfaces $\omega_{i}$ that intersect at edges $e_{i}$. Applying Stokes' theorem to each of these subsurfaces, we find that

$$
\int_{\partial \pi} \boldsymbol{S}^{\alpha} \cdot \boldsymbol{u}_{, \alpha} d a=\sum \int_{\partial \omega_{i}} \boldsymbol{S}^{\alpha} \xi_{(i) \alpha} \cdot \boldsymbol{u} d s-\int_{\partial \pi} \boldsymbol{S}_{\mid \alpha}^{\alpha} \cdot \boldsymbol{u} d a,
$$

where $\xi_{i}=\xi_{(i) \alpha} A^{\alpha}$ is the unit normal to the curve $\partial \omega_{i}$ such that $\left\{\boldsymbol{v}_{i} \boldsymbol{\xi}_{i} \boldsymbol{\tau}_{i}\right\}$ forms a right-handed orthonormal triad, where $\boldsymbol{\tau}_{i}$ is the unit tangent to $\partial \omega_{i}$ and $s$ is arclength measured in the direction of $\boldsymbol{\tau}_{i}$, and where $S_{\mid \alpha}^{\alpha}$ is the covariant divergence on $\partial \pi$, defined by

$$
\boldsymbol{S}_{\mid \alpha}^{\alpha}=A^{-1 / 2}\left(A^{1 / 2} \boldsymbol{S}^{\alpha}\right)_{, \alpha},
$$

with $A=\operatorname{det}\left(\boldsymbol{A}_{\alpha} \cdot \boldsymbol{A}_{\beta}\right)$. It is understood that each curve $\partial \omega_{i}$ in (108) is traversed counterclockwise as the smooth subsurface $\omega_{i}$ is viewed from the side of $\omega_{i}$ into which its surface normal $\boldsymbol{v}_{i}$ is directed. We elaborate further below.

Accordingly (100) is reduced to

$$
\dot{E}^{*}=\int_{\partial \pi}\left[\left(\boldsymbol{T} \boldsymbol{v}-\boldsymbol{S}_{\mid \alpha}^{\alpha}\right) \cdot \boldsymbol{u}+\boldsymbol{S} \boldsymbol{v} \cdot \boldsymbol{u}_{v}\right] d a+\sum \int_{\partial \omega_{i}} \boldsymbol{S} \boldsymbol{\xi}_{i} \cdot \boldsymbol{u} d s-\int_{\pi} \boldsymbol{u} \cdot \operatorname{Div} \boldsymbol{T} d v,
$$


and (96) implies that the virtual power has the form

$$
P_{\pi}=\int_{\partial \pi}\left(\boldsymbol{t} \cdot \boldsymbol{u}+\boldsymbol{s} \cdot \boldsymbol{u}_{v}\right) d a+\sum \int_{e_{i}} \boldsymbol{f}_{i} \cdot \boldsymbol{u} d s+\int_{\pi} \boldsymbol{g} \cdot \boldsymbol{u} d v
$$

where $e_{i}$ is the $i$-th edge of $\partial \pi$.

If no further kinematic constraints are operative, then because $\boldsymbol{u}$ and $\boldsymbol{u}_{v}$ may be specified independently on $\partial \pi$, the fundamental lemma yields the traction and double force

$$
\boldsymbol{t}=\boldsymbol{T} \boldsymbol{v}-\boldsymbol{S}_{\mid \alpha}^{\alpha} \quad \text { and } \quad \boldsymbol{s}=\boldsymbol{S} \boldsymbol{v},
$$

respectively, on $\partial \pi$, and the body force

$$
g=-\operatorname{Div} \boldsymbol{T}
$$

in the interior of $\pi$. By choosing $\boldsymbol{v}=\boldsymbol{L}, \boldsymbol{M}, \boldsymbol{N}$ in succession, we may conclude, from $(112)_{1},(102)$, and (103), that the Lagrange multipliers $\Lambda_{L M}$, etc., are proportional to transverse shear stresses acting on the fiber "cross sections" (see (27)). Similarly, $\Xi_{L M}$, etc., are proportional to transverse double forces.

Concerning the edge forces $f_{i}$, we observe that an edge $e$ is the intersection of two subsurfaces $\omega_{+}$and $\omega_{-}$, say. Accordingly, in (111) $e$ is traversed twice: once in the sense of $\boldsymbol{\tau}_{+}$and once in the sense of $\boldsymbol{\tau}_{-}=-\boldsymbol{\tau}_{+}$. With (112) and (113) in force the fundamental lemma then furnishes the edge force density

$$
\boldsymbol{f}=[\boldsymbol{S} \xi] \text { on } e,
$$

where $[\cdot]$ is the difference of the limits of the enclosed quantity on $e$ when approached from $\omega_{+}$and $\omega_{-}$, i.e, $[\cdot]=(\cdot)_{+}-(\cdot)_{-}$.

Fuller discussions of edge forces, and of the wedge forces operating at vertices in continua of grade higher than two, may be found in [Mindlin 1965; dell'Isola and Seppecher 1995; 1997; dell'Isola et al. 2012; Fosdick 2016].

4.4. Rigid-body variations. In classical rigid-body mechanics the relevant actions are the net force and couple acting on the body. To deduce their forms in the present model, we specialize the virtual-power statement to rigid-body virtual translations and rotations. In view of the invariance of the strain energy under such variations (see (45)), this statement reduces to $P_{\pi}=0$ for all deformations of the form

$$
\chi(X ; \epsilon)=Q(\epsilon) x+d(\epsilon),
$$

where $\boldsymbol{x}=\chi(\boldsymbol{X})$ is an equilibrium deformation field, $\boldsymbol{Q}(\epsilon)$ is a one-parameter family of rotations with $\boldsymbol{Q}(0)=\boldsymbol{I}$, and $\boldsymbol{d}(\epsilon)$ is a family of vectors with $\boldsymbol{d}(0)=\mathbf{0}$. Again using superposed dots to denote derivatives with respect to $\epsilon$, evaluated at $\epsilon=0$, we compute the virtual translational velocity $\boldsymbol{u}(\boldsymbol{X})=\boldsymbol{\omega} \times \boldsymbol{x}+\dot{\boldsymbol{d}}$, where $\boldsymbol{\omega}$ is the axial vector of $\dot{Q}$. 
Because $\boldsymbol{\omega}$ and $\dot{\boldsymbol{d}}$ are arbitrary, the virtual-work statement (see (111)) is satisfied if and only if

$$
\int_{\partial \pi} \boldsymbol{t} d a+\int_{\pi} \boldsymbol{g} d v+\sum \int_{e_{i}} \boldsymbol{f}_{i} d s=\mathbf{0}
$$

and

$$
\int_{\partial \pi}(\boldsymbol{x} \times \boldsymbol{t}+\boldsymbol{c}) d a+\int_{\pi} \boldsymbol{x} \times \boldsymbol{g} d v+\sum \int_{e_{i}} \boldsymbol{x} \times \boldsymbol{f}_{i} d s=\mathbf{0},
$$

where

$$
c=x_{v} \times s
$$

and

$$
\boldsymbol{x}_{v}=\boldsymbol{F} \boldsymbol{v}
$$

is the normal derivative of the equilibrium deformation on $\partial \pi$.

These are respectively the force and moment balances for the arbitrary part $\pi \subset \kappa$ of the body, the latter implying that $c$ is a distribution of couples acting on $\partial \pi$. Because these conditions were derived using a special form of $\boldsymbol{u}$, they are necessary for equilibrium. Indeed, it may be shown that they follow from (112) and (113). However, they are not sufficient - the arbitrariness of $\pi$ notwithstanding - because $(112)_{2}$ involves the entire double force on $\partial \pi$, whereas (118) is insensitive to that part of the double force which is parallel to $\boldsymbol{x}_{v}$. This situation stands in contrast to first-gradient elasticity, in which (116) and (117) (with $c$ and $f_{i}$ equal to zero) are both necessary and sufficient for equilibrium. The utility of the variational approach to our subject can thus hardly be overestimated [Germain 1973]. This perspective is amplified and extended in a recent revival [Eugster and dell'Isola 2017; 2018a; 2018b; Eugster and Glocker 2017] of Hellinger's approach to continuum mechanics.

\section{Example: bending a block to a cylinder}

To illustrate the model we use it to solve the classical problem of bending a block to a cylindrical annulus [Ogden 1984]. The conventional treatment of this problem relies on the use of first-gradient elasticity. Here we highlight the additional flexibility in its solution afforded by the present model.

We choose the fibers to be aligned initially with a Cartesian coordinate system $(X, Y, Z)$, so that

$$
X=X \boldsymbol{L}+Y \boldsymbol{M}+Z \boldsymbol{N} .
$$

The block occupies the volume defined by $A<X<A+W,-H / 2<Y<H / 2$, and $-D / 2<Z<D / 2$, where $A$ is a positive constant, $W$ is the width of the block, $H$ is the height, and $D$ is the depth. The deformed position is

$$
\boldsymbol{x}=\chi(X)=r(X) \boldsymbol{e}_{r}(\theta(Y))+Z N,
$$


where

$$
\boldsymbol{e}_{r}(\theta)=\cos \theta \boldsymbol{L}+\sin \theta \boldsymbol{M} .
$$

Thus, the deformation maps vertical planes $X=$ const. to cylinders $r=$ const., and horizontal planes $Y=$ const. to radial planes $\theta=$ const.. There is no displacement along the $Z$-axis.

The deformation gradient is

$$
\boldsymbol{F}=r^{\prime} \boldsymbol{e}_{r} \otimes \boldsymbol{L}+r \theta^{\prime} \boldsymbol{e}_{\theta} \otimes \boldsymbol{M}+\boldsymbol{N} \otimes \boldsymbol{N},
$$

where $\boldsymbol{e}_{\theta}=\boldsymbol{N} \times \boldsymbol{e}_{r}$, and we assume, as in the classical treatment, that $\theta(Y)=\alpha Y$, with $\alpha$ a positive constant. Accordingly, $\operatorname{det} \boldsymbol{F}=\alpha r r^{\prime}$, and the usual restriction $\operatorname{det} \boldsymbol{F}>0$ implies that $r(X)$ is an increasing function, i.e., $r^{\prime}>0$. It follows immediately that

$$
\boldsymbol{R}=\boldsymbol{e}_{r} \otimes \boldsymbol{L}+\boldsymbol{e}_{\theta} \otimes \boldsymbol{M}+\boldsymbol{N} \otimes \boldsymbol{N} \quad \text { and } \quad \boldsymbol{E}=\boldsymbol{U}=r^{\prime} \boldsymbol{L} \otimes \boldsymbol{L}+\alpha r \boldsymbol{M} \otimes \boldsymbol{M}+\boldsymbol{N} \otimes \boldsymbol{N},
$$

and hence, from (36) and (42), that

$$
\begin{aligned}
\boldsymbol{l} & =\boldsymbol{e}_{r}(\theta), & \boldsymbol{m} & =\boldsymbol{e}_{\theta}(\theta), & \boldsymbol{n} & =\boldsymbol{N}, \\
\lambda_{l} & =r^{\prime}(X), & \lambda_{m} & =\alpha r(X), & \lambda_{n} & =1 .
\end{aligned}
$$

Clearly the rigidity constraints (95) are satisfied.

Using (37)-(39) we find that all fiber twists $\tau_{l, m, n}\left(=\kappa_{(l, m, n) 3}\right)$ vanish. Assuming the fiber constitutive relations $(81)_{2}$, we deduce that

$$
\begin{aligned}
\boldsymbol{R} \boldsymbol{\mu}_{l} & =\boldsymbol{R} \boldsymbol{\mu}_{n}=\mathbf{0}, \\
\boldsymbol{R} \boldsymbol{\mu}_{m} & =\alpha F \boldsymbol{N},
\end{aligned}
$$

so that $\boldsymbol{a}=\mathbf{0}$ in (93) and (103), whereas

$$
\boldsymbol{b}=\alpha F(\boldsymbol{M} \cdot \boldsymbol{v}) \boldsymbol{N}
$$

in (94). Guided by the structure of solutions to rod theory for uniformly curved rods, we seek a solution in which the various $\Lambda$ - the transverse shear stresses acting on the fiber cross sections - vanish. In this case (81) and (103) imply that

$$
\begin{aligned}
\boldsymbol{T}_{l} & =f\left(\lambda_{l}\right) \boldsymbol{e}_{r}, \\
\boldsymbol{T}_{m} & =f\left(\lambda_{m}\right) \boldsymbol{e}_{\theta}, \\
\boldsymbol{T}_{n} & =\mathbf{0},
\end{aligned}
$$

where (see (81)) $f(\lambda)=E(\lambda-1)$. We use (79)-(81) to compute

$$
\operatorname{Div} \boldsymbol{T}=E\left[r^{\prime \prime}-\alpha(\alpha r-1)\right] \boldsymbol{e}_{r},
$$


and conclude, from (113) (with vanishing body force), that $r(X)$ satisfies the simple linear differential equation

$$
r^{\prime \prime}-\alpha^{2} r=-\alpha
$$

the general solution to which is

$$
r(X)=\alpha^{-1}+C_{1} \exp (\alpha X)+C_{2} \exp (-\alpha X),
$$

where $C_{1,2}$ are constants.

To complete the solution we proceed as in the classical case and impose zero traction (and double-force) conditions at $X=A$ and $X=A+W$. With $\boldsymbol{v}= \pm \boldsymbol{L}$ we find that $\boldsymbol{b}$ vanishes. We also assume that the various $\Xi$ vanish on these surfaces, and hence that $S$ also vanishes. The double force then vanishes, as required, and the tractions are $\pm \boldsymbol{T}_{l}$. The condition of zero traction thus requires that $f\left(\lambda_{l}\right)$ vanish, and hence that $r^{\prime}=1$ at $X=A$ and at $X=A+W$. We thus obtain

$$
\begin{aligned}
& C_{1}=\alpha^{-1} \exp (-\alpha B)[1+\exp (-\alpha W)]^{-1}, \\
& C_{2}=-\alpha^{-1} \exp (\alpha B)[1+\exp (\alpha W)]^{-1},
\end{aligned}
$$

where $B=A+W$, and verify that the admissibility condition $r^{\prime}(X)>0$ is satisfied.

On the planes $Z= \pm D / 2$ we again assume that the various $\Xi$ vanish, finding that the tractions and double forces also vanish on these surfaces. From (114) we also find that the edge forces vanish on the edges defined by $(X, Z)=(A, \pm D / 2)$ and $(X, Z)=(A+W, \pm D / 2)$.

The situation is different on the planes $Y= \pm H / 2$. For example, at $Y=H / 2$ we have $\boldsymbol{v}=\boldsymbol{M}$, yielding

$$
\begin{aligned}
\boldsymbol{T} \boldsymbol{v} & =E(\alpha r-1) \boldsymbol{e}_{\theta} \\
\boldsymbol{s} & =\boldsymbol{S}_{m}=\left(r^{\prime} \Xi_{L M}^{+}-F / 2 r\right) \boldsymbol{e}_{r}+\Xi_{M N}^{+} \boldsymbol{N},
\end{aligned}
$$

where the $\Xi^{+}$are the values of the $\Xi$ at $Y=+H / 2$. Further,

$$
\boldsymbol{S}_{\mid \alpha}^{\alpha}=\boldsymbol{S}_{l, X}+\boldsymbol{S}_{n, Z},
$$

where

$$
\begin{aligned}
& \boldsymbol{S}_{l}=\alpha\left(r \Xi_{L M}^{+}+F / 2 r^{\prime}\right) \boldsymbol{e}_{\theta}+\Xi_{L N}^{+} \boldsymbol{N}, \\
& \boldsymbol{S}_{n}=r^{\prime} \Xi_{L N}^{+} \boldsymbol{e}_{r}+\alpha r \Xi_{M N}^{+} \boldsymbol{e}_{\theta} .
\end{aligned}
$$

Then (112) and (128) deliver the traction

$$
\begin{aligned}
\boldsymbol{t}=\left[E(\alpha r-1)-\alpha\left(r \Xi_{L M}^{+}+F / 2 r^{\prime}\right)_{, X}\right] \boldsymbol{e}_{\theta} & \\
& -\Xi_{L N, X}^{+} N-r^{\prime} \Xi_{L N, Z}^{+} \boldsymbol{e}_{r}-\alpha r \Xi_{M N, Z}^{+} \boldsymbol{e}_{\theta} .
\end{aligned}
$$


Accordingly, and in contrast to the classical treatment [Ogden 1984], we may impose a zero-traction condition on this surface, provided that

$$
E\left(r-\alpha^{-1}\right)-\left(r \Xi_{L M}^{+}+F / 2 r^{\prime}\right)_{, X}-r \Xi_{M N, Z}^{+}=0,
$$

together with

$$
\Xi_{L N, X}^{+}=0 \quad \text { and } \quad \Xi_{L N, Z}^{+}=0 .
$$

We assume that $\Xi_{M N, Z}^{+}=0$ and use (130) to reduce (137) to

$$
E \alpha^{-2} r^{\prime \prime}(X)-\left(r \Xi_{L M}^{+}+F / 2 r^{\prime}\right)_{, X}=0,
$$

concluding that

$$
E \alpha^{-2} r^{\prime}-\left(r \Xi_{L M}^{+}+F / 2 r^{\prime}\right)=G^{+}(Z),
$$

for some function $G^{+}$.

The couple distribution $\boldsymbol{c}^{+}$on $Y=H / 2$ is given by (118), with $\boldsymbol{x}_{v}=\boldsymbol{F} \boldsymbol{M}=\alpha \boldsymbol{r} \boldsymbol{e}_{\theta}$. Thus,

$$
\boldsymbol{c}^{+}=\alpha r \boldsymbol{e}_{\theta} \times \boldsymbol{S}_{m}=\alpha r^{\prime}\left(F / 2 r^{\prime}-r \Xi_{L M}^{+}\right) \boldsymbol{N}+\alpha r \Xi_{M N}^{+} \boldsymbol{e}_{r} .
$$

A solution with $\boldsymbol{c}^{+}$parallel to the cylinder axis $N$ and independent of $Z$ is obtained by taking $\Xi_{M N}^{+}=0$ and $G^{+}$to be constant. From $(133)_{2}$ this is seen to be tantamount to the assignment of the double-force distribution on $Y=H / 2$. The edge forces operating at the edges defined by $(X, Y)=(A, H / 2)$ and $(X, Y)=$ $(A+W, H / 2)$ are found, using (114), to be

$$
\boldsymbol{f}=\mp\left[\alpha\left(r \Xi_{L M}^{+}+F / 2\right) \boldsymbol{e}_{\theta}+\Xi_{L N}^{+} N\right],
$$

respectively, where $\Xi_{L M}^{+}$and $\Xi_{L N}^{+}$are evaluated at $X=A, A+W$, respectively, and we have used the condition $r^{\prime}=1$ at both edges. These edge forces vanish if and only if $\Xi_{L N}^{+}$and $r \Xi_{L M}^{+}+F / 2$ vanish at $X=A, A+W$. Combing the latter with (140), we find that

$$
G^{+}=E \alpha^{-2},
$$

and (140) then delivers

$$
\Xi_{L M}^{+}(X)=r^{-1}\left[E \alpha^{-2}\left(r^{\prime}-1\right)-F / 2 r^{\prime}\right]
$$

with $r(X)$ given by (131) and (132). The couple distribution on $Y=H / 2$ is

$$
c^{+}=\left[\alpha F+E \alpha^{-1} r^{\prime}\left(r^{\prime}-1\right)\right] N .
$$

Moreover, because $\Xi_{L N}^{+}$is independent of $X$ (see (138) 1 ), it vanishes everywhere on this surface.

Finally, the edge forces acting on the edges defined by $(Y, Z)=(H / 2, \pm D / 2)$ are found to be

$$
\boldsymbol{f}= \pm \alpha r \Xi_{M N}^{+} \boldsymbol{e}_{\theta},
$$


respectively. These vanish provided that $\Xi_{M N}^{+}$vanishes at $Z= \pm D / 2$. Because this function was assumed to be independent of $Z$ in the course of constructing the solution, it then vanishes everywhere on the plane $Y=H / 2$. We conclude that $S_{n}$ vanishes there, and that $S_{l}$ and the double force are given respectively by

$$
\boldsymbol{S}_{l}=E \alpha^{-1}\left(r^{\prime}-1\right) \boldsymbol{e}_{\theta} \quad \text { and } \quad \boldsymbol{S}_{m}=r^{-1}\left[E \alpha^{-2} r^{\prime}\left(r^{\prime}-1\right)-F\right] \boldsymbol{e}_{r} .
$$

The situation on the surface $Y=-H / 2$ is similar and so we leave the remaining details to the interested reader. A novel feature of the present model is the prediction that the tractions transmitted by the initially vertical fibers to the surfaces $Y= \pm H / 2$ can be nullified by the rigid joints of the intersecting fibers via the associated Lagrange multipliers.

We have said nothing about the stability of this solution. In particular, the vertical fibers near $X=A$ may become susceptible to buckling as the flexure angle $\alpha$ increases. However, we defer the analysis of buckling - of considerable importance in the mechanics of metamaterials - to a future investigation.

\section{References}

[Antman 2005] S. S. Antman, Nonlinear problems of elasticity, 2nd ed., Applied Mathematical Sciences 107, Springer, 2005.

[Barchiesi et al. 2019] E. Barchiesi, M. Spagnuolo, and L. Placidi, "Mechanical metamaterials: a state of the art”, Math. Mech. Solids 24:1 (2019), 212-234.

[dell'Isola and Seppecher 1995] F. dell'Isola and P. Seppecher, "The relationship between edge contact forces, double forces and interstitial working allowed by the principle of virtual power", $C$. $R$. Acad. Sci. II B 321:8 (1995), 303-308.

[dell'Isola and Seppecher 1997] F. dell'Isola and P. Seppecher, "Edge contact forces and quasibalanced power", Meccanica 32:1 (1997), 33-52.

[dell' Isola et al. 2012] F. dell'Isola, P. Seppecher, and A. Madeo, "How contact interactions may depend on the shape of Cauchy cuts in $N$ th gradient continua: approach "à la D'Alembert"', $Z$. Angew. Math. Phys. 63:6 (2012), 1119-1141.

[Dill 1992] E. H. Dill, “Kirchhoff's theory of rods”, Arch. Hist. Exact Sci. 44:1 (1992), 1-23.

[Eremeyev and Pietraszkiewicz 2012] V. A. Eremeyev and W. Pietraszkiewicz, "Material symmetry group of the non-linear polar-elastic continuum”, Int. J. Solids Struct. 49:14 (2012), 1993-2005.

[Eugster and dell'Isola 2017] S. R. Eugster and F. dell'Isola, "Exegesis of the introduction and sect. I from "Fundamentals of the mechanics of continua" by E. Hellinger", Z. Angew. Math. Mech. 97:4 (2017), 477-506.

[Eugster and dell'Isola 2018a] S. R. Eugster and F. dell'Isola, "Exegesis of Sect. II and III.A from "Fundamentals of the mechanics of continua" by E. Hellinger", Z. Angew. Math. Mech. 98:1 (2018), $31-68$.

[Eugster and dell' Isola 2018b] S. R. Eugster and F. dell'Isola, "Exegesis of Sect. III.B from "Fundamentals of the mechanics of continua" by E. Hellinger", Z. Angew. Math. Mech. 98:1 (2018), 69-105. 
[Eugster and Glocker 2017] S. R. Eugster and C. Glocker, "On the notion of stress in classical continuum mechanics”, Math. Mech. Complex Syst. 5:3-4 (2017), 299-338.

[Fosdick 2016] R. Fosdick, "A generalized continuum theory with internal corner and surface contact interactions", Contin. Mech. Thermodyn. 28:1-2 (2016), 275-292.

[Germain 1973] P. Germain, "The method of virtual power in continuum mechanics, 2: Microstructure”, SIAM J. Appl. Math. 25:3 (1973), 556-575.

[Gurtin 1981] M. E. Gurtin, An introduction to continuum mechanics, Mathematics in Science and Engineering 158, Academic, New York, 1981.

[Koiter 1964] W. T. Koiter, "Couple-stresses in the theory of elasticity, I and II", Nederl. Akad. Wetensch. Proc. B 67 (1964), 17-44.

[Landau and Lifshitz 1986] L. D. Landau and E. M. Lifshitz, Theory of elasticity, 3rd ed., Course of Theoretical Physics 7, Elsevier, Oxford, 1986.

[Mieszala et al. 2017] M. Mieszala, M. Hasegawa, G. Guillonneau, J. Bauer, R. Raghavan, C. Frantz, O. Kraft, S. Mischler, J. Michler, and L. Philippe, "Micromechanics of amorphous metal/polymer hybrid structures with 3D cellular architectures: size effects, buckling behavior, and energy absorption capability”, Small 13:8 (2017), art. id. 1602514.

[Mindlin 1965] R. D. Mindlin, "Second gradient of strain and surface-tension in linear elasticity", Int. J. Solids Struct. 1:4 (1965), 417-438.

[Mindlin and Tiersten 1962] R. D. Mindlin and H. F. Tiersten, "Effects of couple-stresses in linear elasticity", Arch. Rational Mech. Anal. 11 (1962), 415-448.

[Neff 2006] P. Neff, "Existence of minimizers for a finite-strain micromorphic elastic solid", Proc. Roy. Soc. Edinburgh Sect. A 136:5 (2006), 997-1012.

[Ogden 1984] R. W. Ogden, Nonlinear elastic deformations, Wiley, New York, 1984.

[Pietraszkiewicz and Eremeyev 2009] W. Pietraszkiewicz and V. A. Eremeyev, "On natural strain measures of the non-linear micropolar continuum”, Int. J. Solids Struct. 46:3-4 (2009), 774-787.

[Reissner 1975] E. Reissner, "Note on the equations of finite-strain force and moment stress elasticity”, Studies in Appl. Math. 54:1 (1975), 1-8.

[Reissner 1987] E. Reissner, "A further note on finite-strain force and moment stress elasticity", $Z$. Angew. Math. Phys. 38:5 (1987), 665-673.

[Spencer and Soldatos 2007] A. J. M. Spencer and K. P. Soldatos, "Finite deformations of fibrereinforced elastic solids with fibre bending stiffness", Int. J. Non-Linear Mech. 42:2 (2007), 355368.

[Steigmann 1996] D. J. Steigmann, "The variational structure of a nonlinear theory for spatial lattices”, Meccanica 31:4 (1996), 441-455.

[Steigmann 2012] D. J. Steigmann, "Theory of elastic solids reinforced with fibers resistant to extension, flexure and twist", Int. J. Non-Linear Mech. 47:7 (2012), 737-742.

[Steigmann 2015] D. J. Steigmann, "Effects of fiber bending and twisting resistance on the mechanics of fiber-reinforced elastomers", pp. 269-305 in Nonlinear mechanics of soft fibrous materials, edited by L. Dorfmann and O. R. W., CISM Courses and Lect. 559, Springer, 2015.

[Toupin 1964] R. A. Toupin, “Theories of elasticity with couple-stress", Arch. Rational Mech. Anal. 17 (1964), 85-112.

[Vangelatos et al. 2019] Z. Vangelatos, K. Komvopoulos, and C. P. Grigoropoulos, "Vacancies for controlling the behavior of microstructured three-dimensional mechanical metamaterials", Math. Mech. Solids 24:2 (2019), 511-524. 
Received 5 Feb 2019. Revised 13 Feb 2019. Accepted 2 Mar 2019.

SIMON R. EUGSTER: eugster@inm.uni-stuttgart.de

Institute for Nonlinear Mechanics, Universität Stuttgart, Stuttgart, Germany

and

International Centre for Mathematics and Mechanics of Complex Systems, Università dell'Aquila, L'Aquila, Italy

FRANCESCO DELL'ISOLA: francesco.dellisola.me@gmail.com

Dipartimento di Ingegneria Strutturale e Geotecnica, Università di Roma "La Sapienza", Roma, Italy

and

International Centre for Mathematics and Mechanics of Complex Systems, Università dell'Aquila, L'Aquila, Italy

DAVID J. STEIGMAnN: dsteigmann@berkeley .edu

Department of Mechanical Engineering, University of California, Berkeley, Berkeley, CA, United States

and

International Centre for Mathematics and Mechanics of Complex Systems, Università dell'Aquila, L'Aquila, Italy 


\section{Guidelines for Authors}

Authors may submit manuscripts in PDF format on-line at the submission page.

Originality. Submission of a manuscript acknowledges that the manuscript is original and and is not, in whole or in part, published or under consideration for publication elsewhere. It is understood also that the manuscript will not be submitted elsewhere while under consideration for publication in this journal.

Language. Articles in MEMOCS are usually in English, but articles written in other languages are welcome.

Required items. A brief abstract of about 150 words or less must be included. It should be selfcontained and not make any reference to the bibliography. If the article is not in English, two versions of the abstract must be included, one in the language of the article and one in English. Also required are keywords and a Mathematics Subject Classification or a Physics and Astronomy Classification Scheme code for the article, and, for each author, postal address, affiliation (if appropriate), and email address if available. A home-page URL is optional.

Format. Authors are encouraged to use $\mathrm{IAT}_{\mathrm{E} X}$ and the standard amsart class, but submissions in other varieties of $\mathrm{T}_{\mathrm{E}} \mathrm{X}$, and exceptionally in other formats, are acceptable. Initial uploads should normally be in PDF format; after the refereeing process we will ask you to submit all source material.

References. Bibliographical references should be complete, including article titles and page ranges. All references in the bibliography should be cited in the text. The use of $\mathrm{BIBT}_{\mathrm{E}} \mathrm{X}$ is preferred but not required. Tags will be converted to the house format, however, for submission you may use the format of your choice. Links will be provided to all literature with known web locations and authors are encouraged to provide their own links in addition to those supplied in the editorial process.

Figures. Figures must be of publication quality. After acceptance, you will need to submit the original source files in vector graphics format for all diagrams in your manuscript: vector EPS or vector PDF files are the most useful.

Most drawing and graphing packages - Mathematica, Adobe Illustrator, Corel Draw, MATLAB, etc. - allow the user to save files in one of these formats. Make sure that what you are saving is vector graphics and not a bitmap. If you need help, please write to graphics@msp.org with as many details as you can about how your graphics were generated.

Bundle your figure files into a single archive (using zip, tar, rar or other format of your choice) and upload on the link you been provided at acceptance time. Each figure should be captioned and numbered so that it can float. Small figures occupying no more than three lines of vertical space can be kept in the text ("the curve looks like this:"). It is acceptable to submit a manuscript with all figures at the end, if their placement is specified in the text by means of comments such as "Place Figure 1 here". The same considerations apply to tables.

White Space. Forced line breaks or page breaks should not be inserted in the document. There is no point in your trying to optimize line and page breaks in the original manuscript. The manuscript will be reformatted to use the journal's preferred fonts and layout.

Proofs. Page proofs will be made available to authors (or to the designated corresponding author) at a Web site in PDF format. Failure to acknowledge the receipt of proofs or to return corrections within the requested deadline may cause publication to be postponed. 
Mathematics and Mechanics of Complex Systems vol. 7 no. 1

Homogenization of nonlinear inextensible pantographic structures by $\Gamma$-convergence

Jean-Jacques Alibert and Alessandro Della Corte A note on Couette flow of micropolar fluids according to Eringen's theory

Wilhelm Rickert, Elena N. Vilchevskaya and Wolfgang H.

Müller

Analytical solutions for the natural frequencies of rectangular symmetric angle-ply laminated plates

Florence Browning and Harm Askes

On the blocking limit of steady-state flow of Herschel-Bulkley fluid

Farid Messelmi

Continuum theory for mechanical metamaterials with a cubic lattice substructure

Simon R. Eugster, Francesco dell' Isola and David J.

Steigmann

MEMOCS is a journal of the International Research Center for the Mathematics and Mechanics of Complex Systems at the Università dell' Aquila, Italy.

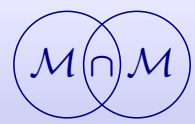

